\title{
Stable isotope record of the terminal Neoproterozoic Krol platform in the Lesser Himalayas of northern India
}

\author{
Alan J. Kaufman ${ }^{\mathrm{a}, *}$, Ganqing Jiang ${ }^{\mathrm{b}}$, Nicholas Christie-Blick ${ }^{\mathrm{c}}$, \\ Dhiraj M. Banerjee ${ }^{\mathrm{d}}$, Vibhuti Rai ${ }^{\mathrm{e}}$ \\ a University of Maryland, Geology Department, College Park, MD 20742-4211, United States \\ ${ }^{\mathrm{b}}$ University of Nevada, Department of Geosciences, Las Vegas, NV 89154-4010, United States \\ ${ }^{\mathrm{c}}$ Department of Earth and Environmental Sciences, Lamont-Doherty Earth Observatory of Columbia University, \\ Palisades, NY 10964-8000, United States \\ ${ }^{\mathrm{d}}$ Department of Geology, University of Delhi, Delhi 110007, India \\ e Department of Geology, University of Lucknow, Lucknow 226007, India
}

Received 18 October 2005; received in revised form 16 February 2006; accepted 27 February 2006

\begin{abstract}
The terminal Neoproterozoic succession in the Lesser Himalaya of India, including the Infra Krol Formation and Krol Group, represent the thickest known accumulation of carbonate strata of this time period, and hence is an ideal target for chemostratigraphic and sequence stratigraphic investigation. High-resolution analyses reveal several negative $\delta^{13} \mathrm{C}$ excursions. Only one of these is related to known Neoproterozoic ice ages, and many vary in amplitude and stratigraphic position in an oblique regional transect of the platform. A combination of stratigraphic and geochemical tests, and comparison with sections elsewhere, leads to an evaluation of the relative contributions of primary and secondary signals. Two excursions are associated with abrupt facies changes associated with shallowing and accumulation of carbonate in organic-rich restricted environments, or with karstification of a subaerially exposed platform; these are currently interpreted to be diagenetic artifacts. Other negative $\delta^{13} \mathrm{C}$ excursions are recorded in open marine transgressive facies, and are interpreted to represent biogeochemical anomalies of global significance. The magnitude of these excursions, however, is complicated by their lateral inconsistency between adjacent sections. Two interpretations are proposed. One possibility is that the observed isotopic inconsistency is due to diagenesis not revealed by the application of existing geochemical criteria. A composite $\delta^{13} \mathrm{C}$ curve constructed under this assumption is broadly consistent with that documented elsewhere, and may reflect modest biogeochemical changes of global scale. An alternative interpretation is that the inconsistency of $\delta^{13} \mathrm{C}$ values relates to stratigraphic hiatus between correlated horizons, incomplete sampling, and/or lack of appropriate carbonates facies. The composite $\delta^{13} \mathrm{C}$ curve constructed under this assumption shows large-magnitude (up to $15 \%$ ) negative $\delta^{13} \mathrm{C}$ anomalies that are taken to imply remarkable perturbations of ocean geochemistry and the episodic input of ${ }^{13} \mathrm{C}$-depleted alkalinity during terminal Neoproterozoic time.
\end{abstract}

(C) 2006 Elsevier B.V. All rights reserved.

Keywords: Neoproterozoic; Krol Group; Isotope

\footnotetext{
* Corresponding author. Tel.: +1 301405 0395; fax: +1 3014053597 .

E-mail address: kaufman@geol.umd.edu (A.J. Kaufman).
}

\section{Introduction}

Neoproterozoic successions worldwide are characterized by prominent carbon and sulfur isotope anomalies, in many cases associated with glaciogenic strata, that record perturbations in the biogeochemical cycling of these elements. Insofar as isotope event stratigraphy 
reflects environmental change, charting the full range of chemical variability in a well defined physical stratigraphic framework is important for the understanding of early animal evolution (e.g., Kaufman et al., 1991; Hoffman et al., 1998; Jacobsen and Kaufman, 1999; Knoll, 2000).

Stratigraphic limitations in the abundance and distribution of carbonate through siliciclastic-dominated successions, diagenetic alteration of depositional $\delta^{13} \mathrm{C}$ compositions, and facies-restricted anomalies (Kaufman et al., 1991) may each obscure the record of biogeochemical phenomena. This is especially true for studies that rely on data from a single section. These uncertainties together represent the most significant hurdle in seeking links between geochemical perturbations and their biological context at the end of the Proterozoic Eon.

Recent reports from a variety of terminal Neoproterozoic successions reveal the presence of unusually large negative carbon isotope anomalies down to a nadir of $-10 \%$ or more (Burns and Matter, 1993; Kimura et al., 1997; Calver, 2000; Amthor et al., 2003; Corsetti and Kaufman, 2003; Xiao et al., 2004; Kaufman et al., in press), which are apparently unrelated to global scale glaciation. The origin (or origins) of these largemagnitude events is likely to be different from those proposed for the post-glacial "cap carbonates" (Kaufman et al., 1991, 1997; Hoffman et al., 1998; Kennedy et al., 2001; Hoffman and Schrag, 2002; Jiang et al., 2003a).

To obtain as complete as possible an isotopic record of terminal Neoproterozoic time, we conducted an inte- grated sequence and chemostratigraphic study of the Krol platform of northern India. This platform was chosen for study as it stands out as one of the thickest accumulations of carbonate strata of this time, and is amenable for both physical stratigraphic study and isotopic analysis. Earlier reports of carbon and oxygen isotope analyses of Krol carbonates can be found in Aharon et al. (1987) and Banerjee et al. (1997). We report here high-resolution isotopic analysis of multiple sections across the platform interior to outer shelf within a sequence stratigraphic framework and discuss alternative reconstructions of temporal changes in the carbon isotopic composition of terminal Neoproterozoic oceans.

\section{Geological background}

Neoproterozoic strata in northern India crop out in a series of doubly plunging synclines in the Lesser Himalaya thrust belt (Fig. 1). The succession encompassing the Krol Group consists of three distinct intervals deposited in a north- to northwest-facing rift to passive margin (Fig. 2; Jiang et al., 2003b) including, (1) preglacial siliciclastic rocks (Jaunsar and Simla groups), (2) two levels of glacial diamictite separated by siltstone and sandstone that are assigned to the Blaini Formation, and (3) post-glacial siliciclastic and carbonate rocks of the Infra Krol Formation and Krol Group.

The timing of the rift-to-passive margin transition is not well established, but is interpreted to be within or at

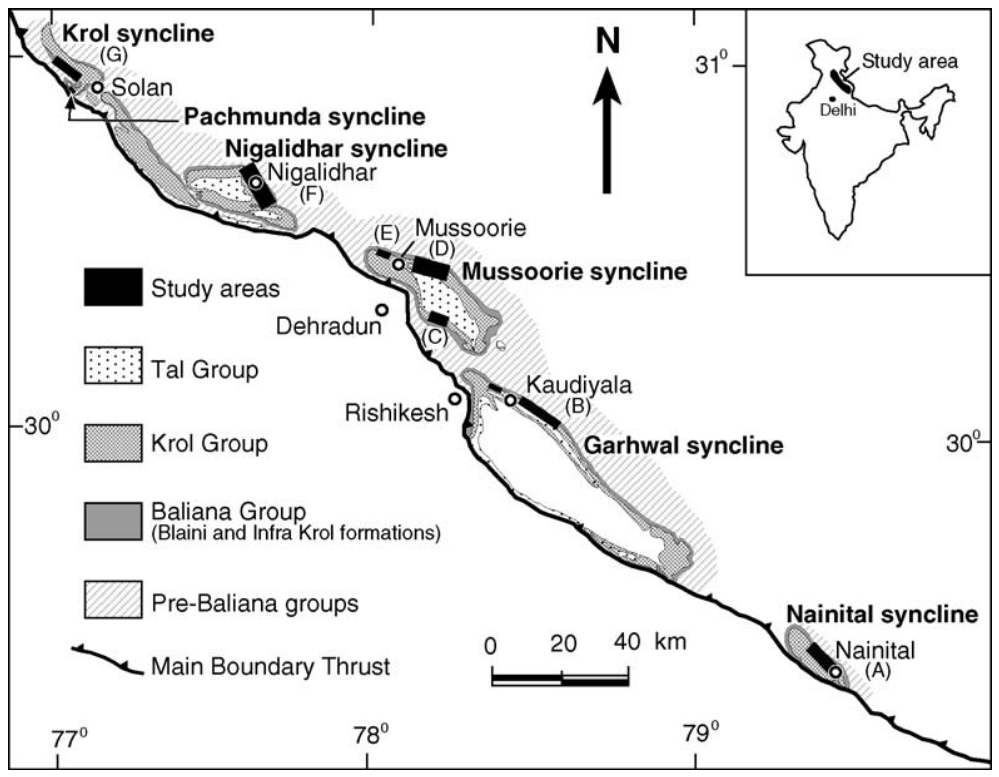

Fig. 1. Simplified geological map of the five studied synclines showing outcrops and measured sections in the Lesser Himalaya, northern India (after Singh and Rai, 1983). 


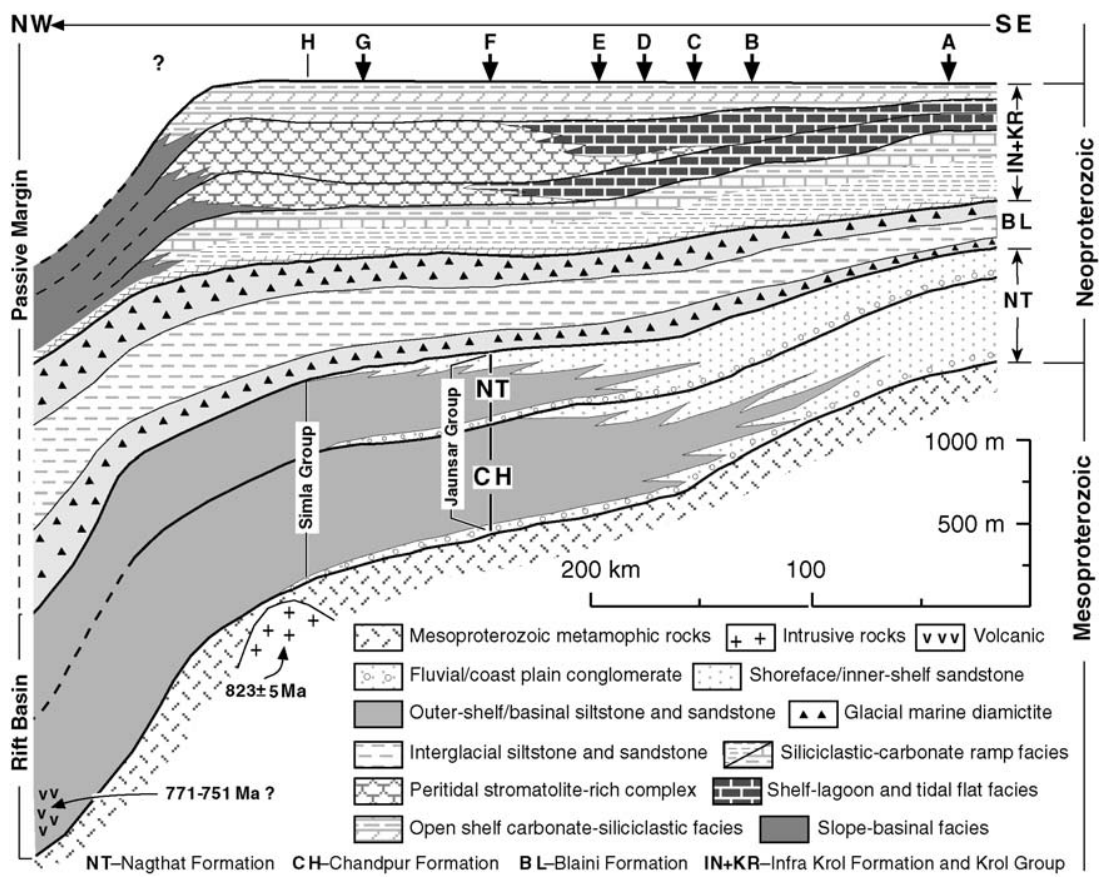

Fig. 2. Neoproterozoic stratigraphy and basin evolution of the Lesser Himalaya, northern India. Measured sections A through G are located in Fig. 1 (after Jiang et al., 2003b). The stratigraphy of section H (Simla) is summarized from Valdiya (1970) and Kumar and Brookfield (1987).

the base of the glaciogenic units, based on the absence of igneous rocks and comparatively simple thickness and facies trends across the basin. Furthermore, there is no evidence for substantial syn-depositional tectonism, which would be expected in a foreland basin (Jiang et al., 2002, 2003b).

Terminal Neoproterozoic strata include the Infra Krol Formation and the Krol Group (Fig. 3A), which overlie the Blaini Formation, the upper part of which has been inferred to relate to Marinoan (early Varanger) glaciation ca. $635 \mathrm{Ma}$ (Jiang et al., 2003c; Condon et al., 2005; Zhang et al., 2005). The whole of the succession above this diamictite encompasses the newly-ratified Ediacaran Period of Earth history (Knoll et al., 2004), which is currently constrained to extend from ca. $635 \mathrm{Ma}$ (Condon et al., 2005) to the Precambrian-Cambrian boundary at $542 \mathrm{Ma}$ (Bowring et al., 1993; Grotzinger et al., 1995; Amthor et al., 2003).

Immediately above the upper Blaini diamictite is a 5-15-m-thick cap carbonate consisting of thinly laminated and iron-rich muddy dolomite, the upper part of which is parted by thin shale. The Blaini cap carbonate is only locally exposed and the unusual sedimentary structures and textures (e.g., tepee-like structures, sheet cracks, tubestones, and seafloor cements) characteristic of other cap carbonates globally (e.g., Kennedy, 1996; Hoffman et al., 1998; James et al., 2001; Jiang et al., 2003a) have not been observed in the available outcrops. The cap carbonate is overlain by up to $250 \mathrm{~m}$ of shale, calcareous siltstone and fine-grained sandstone of the Infra Krol Formation. A sandstone up to $60 \mathrm{~m}$ thick (Fig. 3A) is locally present at the top of the Infra Krol Formation at Krol and Nainital synclines (Fig. 3B). This sandstone unit is commonly capped by $<2 \mathrm{~m}$ thick, silty and muddy dolomite with occasional phosphorite intraclasts.

Overlying the Infra Krol shale and siltstone is the Krol Group, which is subdivided into five informal units designated Krol A through E (Fig. 3A). Krol A is characterized by greenish to gray, micritic limestone and shale with chertified phosphate nodules containing cyanobacterial fossils (Tiwari and Knoll, 1994). Krol B stands out as an unusual red siltstone interlayered with thin gray to white dolomite; desiccation cracks and gyspsum casts have been reported in the Krol B, suggesting shallow water deposition (Jiang et al., 2002, 2003b). The Krol $\mathrm{B}$ is overlain by interbedded black shale and limestone of the lower Krol C, which is of considerable thickness in the most proximal section (Nainital syncline; loc. A in Fig. 3B). The upper part of Krol C through Krol D consists of thick ( $>500 \mathrm{~m}$ ) microbial dolomite with abundant stromatolites in the western synclines (Nigalidhar and Krol synclines; loc. F and $\mathrm{G}$ in Fig. 3B, respectively), passing towards the southeast into interlayered 


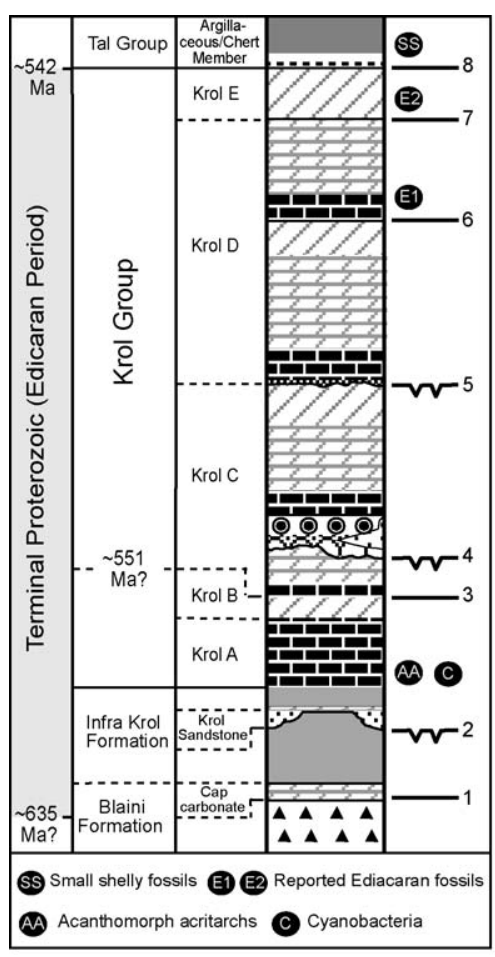

(A)

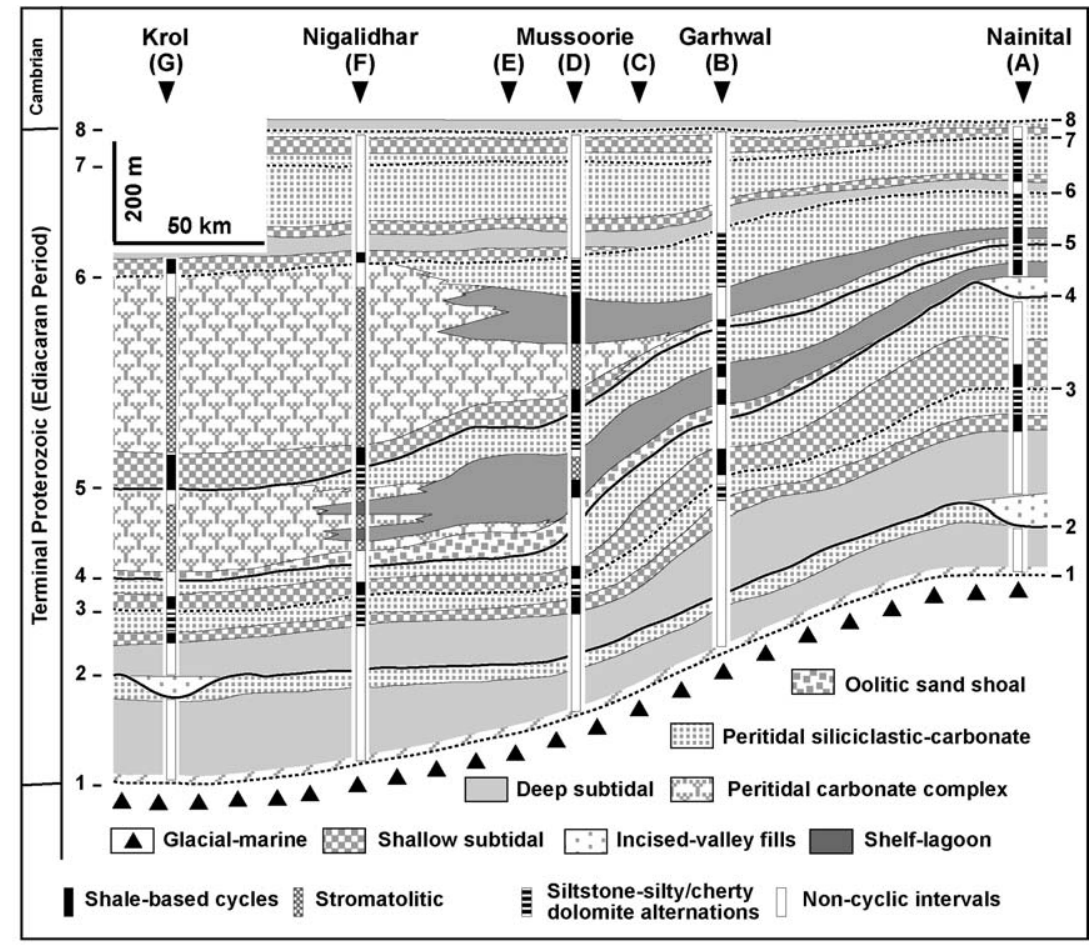

(B)

Fig. 3. (A) Generalized terminal Neoproterozoic stratigraphy of the Infra Krol Formation and Krol Group showing the stratigraphic nomenclature, reported fossil horizons, and regional stratigraphic discontinuities. (B) Interpretation of facies associations across the Krol platform from southeast (Nainital syncline) to northwest (Krol syncline). After Jiang et al. (2003b).

microbial dolomite and shaly limestone interpreted to represent a shelf lagoon setting (loc. A-F in Fig. 3B). The uppermost part of the $\mathrm{Krol}$ (Krol E) is mainly muddy dolomite, and is overlain by the siliciclastic-dominated Tal Group. The basal unit of the Tal contains condensed black shale, phosphate, chert, and cherty dolomite from which small shelly fossils of Early Cambrian aspect have been reported (Singh and Rai, 1983; Shanker and Mathur, 1992).

Sequence stratigraphic investigations reveal eight facies associations separated by regional stratigraphic discontinuities that have been traced along the Lesser Himalaya thrust belt (Fig. 3B; Jiang et al., 2002, 2003b). Three of the surfaces are interpreted as sequence boundaries on the basis of (1) locally developed incised valleys $<60 \mathrm{~m}$ deep, (2) paleokarst with $<50 \mathrm{~m}$ of mappable relief, and (3) the presence of breccia and calcrete filling vertical fissures, dikes, cavities, and shallow depressions in underlying carbonate rocks. The remaining discontinuities are regional flooding surfaces identified on the basis of a transition in facies stacking patterns, typically in a forestepping to backstepping motif, with abrupt upward deepening above each surface. Such a stratigraphic framework provides the basis for sampling in multiple sections, and for the comparisons of isotopic values in laterally variable facies belts across the synclines.

\section{Methods}

For this study, 822 carbonate samples from a variety of cyclic sedimentary facies (Jiang et al., 2003b) were collected at high stratigraphic resolution through the thick Infra Krol Formation and Krol Group, in multiple sections from five widely separated synclines (Table 1). Following petrographic characterization, micro-samples were carefully drilled from the finest-grained portions of these rocks (Kaufman et al., 1991; Kaufman and Knoll, 1995; Corsetti and Kaufman, 2003), and resulting powders were used for subsequent elemental and isotopic analysis.

For elemental analyses, 5-10 mg of sample powder was digested in $0.5 \mathrm{M}$ acetic acid, centrifuged to isolate silicate minerals, and the supernant diluted to $10 \mathrm{ml}$ with ultra-pure Milli-Q water. Solutions were analyzed for $\mathrm{Sr}$ and $\mathrm{Mn}$ abundances with an atomic absorption spectrophotometer at the University of Maryland. Uncertainties determined by the repeated measurement of a 
Table 1

Lithologic, elemental and isotopic data of samples from the terminal Neoproterozoic Infra Krol Formation and Krol Group from the Lesser Himalaya, northern India

\begin{tabular}{|c|c|c|c|c|c|c|c|c|c|c|}
\hline $\begin{array}{l}\text { Sample } \\
\text { number }\end{array}$ & $\begin{array}{l}\text { Position } \\
\text { (m) }\end{array}$ & Lithology* & $\begin{array}{l}\delta^{13} \mathrm{C} \\
(\% \circ, \mathrm{PDB})\end{array}$ & $\begin{array}{l}\delta^{18} \mathrm{O} \\
(\% \circ, \mathrm{PDB})\end{array}$ & $\begin{array}{l}\mathrm{Sr} \\
(\mathrm{ppm})\end{array}$ & $\begin{array}{l}\mathrm{Mn} \\
(\mathrm{ppm})\end{array}$ & $\begin{array}{l}\mathrm{Mn} / \\
\mathrm{Sr}\end{array}$ & $\begin{array}{l}\text { TOC } \\
(\mathrm{mg} / \mathrm{g})\end{array}$ & $\begin{array}{l}\delta^{13} \mathrm{C}_{\mathrm{TOC}} \\
(\%, \mathrm{PDB})\end{array}$ & $\begin{array}{l}\text { Stratigraphi } \\
\text { units }\end{array}$ \\
\hline \multicolumn{11}{|c|}{ 1. Samples from Nainital syncline } \\
\hline \multicolumn{11}{|l|}{ NR } \\
\hline NR-1 & 7.0 & $\mathrm{ch} / \mathrm{silt}$ dol & 2.89 & -7.71 & & & & 0.15 & -21.7 & Krol B \\
\hline NR-2 & 11.0 & $\mathrm{ch} / \mathrm{silt}$ dol & 2.65 & -7.62 & & & & 0.16 & -22.2 & \\
\hline NR-3 & 14.0 & $\mathrm{ch} / \mathrm{silt}$ dol & 3.19 & -8.23 & & & & 0.09 & -19.8 & \\
\hline NR-4 & 18.5 & $\mathrm{ch} / \mathrm{silt}$ dol & 2.9 & -7.75 & & & & 0.09 & -19.1 & \\
\hline NR-5 & 22.5 & $\mathrm{ch} / \mathrm{silt}$ dol & & & 88.14 & 6433.64 & 72.99 & & & \\
\hline NR-6 & 25.0 & $\mathrm{ch} / \mathrm{silt}$ dol & 1.53 & -8.82 & & & & 0.7 & -25.6 & \\
\hline NR-7 & 30.5 & $\mathrm{ch} / \mathrm{silt}$ dol & -1.49 & -7.69 & & & & 0.37 & -21.1 & \\
\hline NR-8 & 31.5 & $\mathrm{ch} / \mathrm{silt}$ dol & -1.18 & -7.44 & & & & 0.13 & -20.0 & \\
\hline NR-9 & 34.5 & $\mathrm{ch} / \mathrm{silt}$ dol & -2.35 & -6.71 & & & & 0.21 & -21.3 & \\
\hline NR-10 & 38.5 & $\mathrm{ch} / \mathrm{silt}$ dol & -1.55 & -8.79 & & & & 0.14 & -20.5 & \\
\hline NR-11 & 39.5 & $\mathrm{ch} / \mathrm{silt}$ dol & -9 & -8.95 & & & & 0.09 & -14.6 & \\
\hline NR-12 & 42.5 & $\mathrm{ch} / \mathrm{silt}$ dol & -8.34 & -9.43 & & & & 0.71 & -24.8 & \\
\hline NR-13 & 46.0 & $\mathrm{ch} / \mathrm{silt}$ dol & -8.43 & -8.89 & & & & 0.14 & -20.9 & Surface 3 \\
\hline NR-14 & 53.0 & $1 \mathrm{~m}$ mudst & -7.95 & -12.93 & 46.81 & 714.86 & 15.27 & 0.11 & -19.9 & \\
\hline NR-15 & 61.5 & $1 \mathrm{~m}$ mudst & -6.13 & -13.65 & 23.44 & 376.62 & 16.07 & 0.12 & -21.9 & \\
\hline NR-16 & 72.0 & pel packst & -9.21 & -15.31 & 49.38 & 839.8 & 17.01 & 0.11 & -21.0 & \\
\hline NR-17 & 79.5 & mud dol & -5.64 & -11.46 & & 1153.74 & & 0.21 & -21.4 & \\
\hline NR-18 & 89.5 & mud dol & -11.82 & -17.48 & 24.83 & 441.42 & 17.78 & 0.14 & -20.5 & \\
\hline NR-19 & 93.5 & pel packst & -7.38 & -10.75 & & & & 2.04 & -33.8 & \\
\hline NR-20 & 101.5 & pel packst & -6.92 & -11.78 & 22.05 & 735.61 & 33.37 & 0.17 & -23.9 & \\
\hline NR-21 & 108.5 & org sh & & & & 174.54 & & & & \\
\hline NR-22 & 118.0 & org sh & -6.18 & -9.3 & 23.34 & 192.01 & 8.23 & & & \\
\hline NR-23 & 128.0 & org sh/lm & -6.85 & -9.35 & & & & 2.32 & -32.0 & \\
\hline NR-24 & 137.0 & org sh & -3.26 & -7.25 & & & & & & \\
\hline NR-25 & 142.5 & org sh & -3.12 & -10.2 & & & & & & Krol C \\
\hline NR-26 & 145.5 & lm mudst & -0.89 & -15.72 & 113.1 & 366.66 & 3.24 & & & \\
\hline NR-27 & 150.0 & lm mudst & 0.2 & -15.87 & & & & 0.2 & -25.6 & \\
\hline NR-28 & 161.5 & mic dol & 0.95 & -8.2 & & & & 0.04 & & \\
\hline NR-29 & 171.0 & mic dol & & & & & & 0.22 & -26.9 & \\
\hline NR-30 & 173.0 & ch dol & 1.23 & -9.85 & & & & 0.39 & -26.8 & \\
\hline NR-31 & 176.0 & mic dol & 1.49 & -7.11 & & & & 0.3 & -29.3 & \\
\hline NR-32 & 180.0 & ch dol & 1.44 & -8.23 & & & & 0.53 & -28.0 & \\
\hline NR-33 & 188.0 & mic dol & 1.69 & -14.24 & 21.01 & 632.44 & 30.1 & & & \\
\hline NR-34 & 197.5 & silt/sand dol & 1.64 & -13.99 & 26.54 & 599.24 & 22.56 & & & \\
\hline NR-35 & 209.0 & silt/ch dol & 1.19 & -9.14 & 47.49 & 1410.34 & 29.7 & & & \\
\hline NR-36 & 223.0 & silt/ch dol & 2.54 & -3.41 & & & & & & Surface 4 \\
\hline NR-37 & 238.5 & karstic br & 1.5 & -7.43 & 52.66 & 3947.11 & 74.95 & & & \\
\hline NR-40 & 295.0 & mud dol & 3.77 & -8.91 & 88.83 & 912.48 & 10.27 & & & \\
\hline NR-41 & 300.0 & dol wackest & 5.35 & -4.78 & 76.53 & 462.83 & 6.05 & & & \\
\hline NR-42 & 305.0 & mic dol & 5.01 & -4.63 & & & & & & Krol C \\
\hline NR-43 & 318.0 & mic dol & 5.62 & -2.62 & 77.13 & 331.3 & 4.3 & & & \\
\hline NR-44 & 329.0 & Wackestone & 3.3 & -6.14 & 76.1 & 145.57 & 1.91 & & & \\
\hline NR-45 & 337.0 & $\begin{array}{l}\operatorname{lm} \\
\text { mudst/wackest }\end{array}$ & 6.74 & -6.3 & & & & & & \\
\hline NR-46 & 341.0 & silt dol & 7.82 & -2.84 & & & & & & \\
\hline NR-47 & 348.0 & mic dol & 8.03 & -4.5 & 49.75 & 131.08 & 2.64 & & & \\
\hline NR-48 & 351.0 & $\mathrm{br} / \mathrm{ch}$ dol & 6.75 & -4.62 & 23.75 & 107.15 & 4.51 & & & Surface 5 \\
\hline NR-49 & 357.0 & mic dol & 7.36 & -8.01 & 28.59 & 342.01 & 11.96 & & & \\
\hline NR-50 & 361.0 & mic dol & 6.96 & -11.37 & & & & & & \\
\hline NR-51 & 363.0 & mud dol & 0.9 & -4.45 & & & & & & \\
\hline NR-52 & 367.0 & dol wackest & 3.61 & -8.91 & 91.39 & 504.42 & 5.52 & & & \\
\hline NR-53 & 373.0 & mic dol & 4.16 & -11.2 & 17.97 & 221.1 & 12.31 & & & \\
\hline NR-54 & 381.0 & intra grainst & 3.47 & -11.04 & & & & & & \\
\hline
\end{tabular}


Table 1 (Continued)

\begin{tabular}{|c|c|c|c|c|c|c|c|c|c|}
\hline $\begin{array}{l}\text { Sample } \\
\text { number }\end{array}$ & $\begin{array}{l}\text { Position } \\
(\mathrm{m})\end{array}$ & Lithology & $\begin{array}{l}\delta^{13} \mathrm{C} \\
(\% \circ, \mathrm{PDB})\end{array}$ & $\begin{array}{l}\delta^{18} \mathrm{O} \\
(\% \circ, \mathrm{PDB})\end{array}$ & $\begin{array}{l}\mathrm{Sr} \\
(\mathrm{ppm})\end{array}$ & $\begin{array}{l}\mathrm{Mn} \\
(\mathrm{ppm})\end{array}$ & $\begin{array}{l}\mathrm{Mn} / \\
\mathrm{Sr}\end{array}$ & $\begin{array}{l}\delta^{13} \mathrm{C}_{\mathrm{TOC}} \\
(\% o, \mathrm{PDB})\end{array}$ & $\begin{array}{l}\text { Stratigraphic } \\
\text { units }\end{array}$ \\
\hline NR-55 & 391.0 & intra grainst & 4.37 & -11.83 & 128.77 & 336.15 & 2.61 & & Krol D? \\
\hline NR-56 & 405.0 & mic dol & 4.44 & -12.02 & 22.83 & 315.89 & 13.84 & & \\
\hline NR-57 & 409.0 & pel packst & 3.62 & -6.06 & & & & & \\
\hline NR-58 & 417.0 & mic dol & 4.84 & -3.49 & & & & & \\
\hline NR-59 & 428.0 & mic dol & 4.83 & -3.31 & & & & & \\
\hline NR-60 & 433.0 & $\mathrm{ch} / \mathrm{silt}$ dol & 4.64 & -6.15 & & & & & \\
\hline NR-61 & 440.0 & $\mathrm{ch} / \mathrm{silt}$ dol & 5.56 & -1.93 & & & & & \\
\hline NR-62 & 453.0 & $\mathrm{ch} / \mathrm{silt}$ dol & 3.06 & -1.3 & & & & & Surface 6 \\
\hline NR-63 & 485.0 & dol wackest & 4.8 & -8.78 & & & & & \\
\hline NR-64 & 495.0 & $\mathrm{ch} /$ silt dol & 3.71 & -3.73 & 53.52 & & & & \\
\hline NR-65 & 503.0 & mic dol & 5.28 & -2.42 & 54.05 & 139.4 & 2.58 & & \\
\hline NR-66 & 516.0 & $\mathrm{ch} / \mathrm{silt}$ dol & 6.98 & -3.81 & 102.19 & 388.6 & 3.8 & & \\
\hline NR-67 & 528.0 & mic dol & 3.29 & -3.84 & & & & & \\
\hline NR-68 & 536.0 & silt/ch dol & 3.01 & -4.6 & & & & & Krol D? \\
\hline NR-69 & 549.0 & silt/ch dol & 3.14 & -4.03 & & & & & \\
\hline NR-70 & 561.0 & mic dol & 4.32 & -5.4 & & & & & \\
\hline NR-71 & 568.0 & silt/ch dol & 3.09 & -1.17 & & & & & \\
\hline NR-72 & 573.0 & silt/ch dol & 2.15 & -1.77 & 77.92 & 1046.39 & 13.43 & & \\
\hline NR-73 & 581.0 & silt/ch dol & 3.94 & -1.87 & 74.91 & 323.78 & 4.32 & & \\
\hline NR-74 & 592.0 & silt/ch dol & 2.76 & -4.57 & 47.51 & 1945.7 & 40.96 & & \\
\hline NR-75 & 605.0 & silt/ch dol & 3.07 & -3.13 & 70.22 & 557.58 & 7.94 & & Surface 7 \\
\hline NR-76 & 612.0 & mud dol & -0.09 & -14.11 & 27.29 & 1540.32 & 56.45 & & Krol E? \\
\hline NR-77 & 625.0 & mud dol & 2.49 & -3.4 & & & & & \\
\hline \multicolumn{10}{|l|}{ NB-NC } \\
\hline NB-1 & 544.0 & lm mudst & 2.83 & -12.78 & & & & & \\
\hline NB-2 & 553.0 & mud lm & 3.62 & -13.32 & & & & & \\
\hline NB-3 & 564.0 & lm mudst & 1.88 & -13.14 & & & & & \\
\hline NB-4 & 591.0 & $1 \mathrm{~m}$ mudst & -0.24 & -12.71 & 341.02 & 1368.41 & 4.01 & & \\
\hline NB-5 & 603.0 & lm mudst & 1.27 & -13.1 & & & & & Krol A \\
\hline NB-6 & 628.0 & mud lm & -1.47 & -12.51 & & & & & \\
\hline NC-1 & 650.0 & lm mudst & -5.41 & -6.19 & 67.76 & 2528.5 & 37.32 & & \\
\hline $\mathrm{NC}-2$ & 654.0 & mud $1 \mathrm{~m}$ & -0.29 & -11.82 & & & & & \\
\hline $\mathrm{NC}-3$ & 662.0 & intra grainst & 1.5 & -13.47 & 536.7 & 898.79 & 1.68 & & \\
\hline $\mathrm{NC}-4$ & 667.0 & intra grainst & 4.07 & -13.16 & 351.49 & 324.57 & 0.92 & & \\
\hline $\mathrm{NC}-5$ & 674.0 & intra grainst & 3.09 & -12.68 & 390.29 & 534.05 & 1.37 & & \\
\hline NC-6 & 682.0 & silt/ch dol & 2.92 & -9.44 & 255.15 & 1693.07 & 6.64 & & Krol B \\
\hline NC-7 & 689.0 & silt/ch dol & 2.78 & -6.23 & & & & & \\
\hline NC-8 & 695.0 & silt/ch dol & 3.15 & -7.65 & & & & & \\
\hline \multirow{2}{*}{\multicolumn{10}{|c|}{$\begin{array}{l}\text { 2. Samples from Garhwal syncline } \\
\text { k97.1 }\end{array}$}} \\
\hline & & & & & & & & & \\
\hline k97.1.0.0 & 223.0 & mud dol & -2.35 & -10.79 & 142.07 & 2459.05 & 17.31 & & Surface 1 \\
\hline k97.1.0.5 & 223.5 & mud dol & -2.35 & -9.75 & & & & & \\
\hline k97.1.1.0 & 224.0 & mud dol & -2.23 & -9.93 & 60.71 & 1217.26 & 20.05 & & \\
\hline k97.1.1.5 & 224.5 & mud dol & -2.37 & -10.47 & 63.02 & 1392.34 & 22.09 & & \\
\hline $\mathrm{k} 97.1 .2 .0$ & 225.0 & mud dol & -2.24 & -10.58 & 41.26 & 1261.01 & 30.56 & & \\
\hline $\mathrm{k} 97.1 .2 .5$ & 225.5 & mud dol & -2.73 & -10.98 & 63.31 & 1751.73 & 27.67 & & \\
\hline k97.1.3.0 & 226.0 & mud dol & -2.26 & -10.39 & 69.27 & 1705.03 & 24.61 & & \\
\hline $\mathrm{k} 97.1 .3 .5$ & 226.5 & mud dol & -2.23 & -11.27 & 103.79 & & & & \\
\hline k97.1.4.0 & 227.0 & mud dol & -0.61 & -12.11 & & & & & $\begin{array}{l}\text { "Cap } \\
\text { carbonate" }\end{array}$ \\
\hline k97.1.4.5 & 227.5 & mud dol & -0.91 & -12.49 & 23.21 & 287.94 & 12.4 & & \\
\hline k97.1.5.0 & 228.0 & mud dol & -0.92 & -12.82 & 21.56 & 265.35 & 12.31 & & \\
\hline k97.1.5.5 & 228.5 & mud dol & -0.49 & -12.28 & & 253.79 & & & \\
\hline k97.1.6.0 & 229.0 & mud dol & -0.29 & -11.91 & 21.86 & 200.47 & 9.17 & & \\
\hline k97.1.6.5 & 229.5 & mud dol & -0.84 & -12.33 & & & & & \\
\hline $\mathrm{k} 97.1 .7 .0$ & 230.0 & mud dol & -0.36 & -12.32 & & & & & \\
\hline $\mathrm{k} 97.1 .7 .5$ & 230.5 & mud dol & 0.04 & -11.88 & 22.2 & 187.85 & 8.46 & & \\
\hline k97.1.8.0 & 231.0 & mud dol & -2.31 & -11.06 & & & & & \\
\hline
\end{tabular}


Table 1 (Continued)

\begin{tabular}{|c|c|c|c|c|c|c|c|c|c|}
\hline $\begin{array}{l}\text { Sample } \\
\text { number }\end{array}$ & $\begin{array}{l}\text { Position } \\
\text { (m) }\end{array}$ & Lithology* & $\begin{array}{l}\delta^{13} \mathrm{C} \\
(\% \circ, \mathrm{PDB})\end{array}$ & $\begin{array}{l}\delta^{18} \mathrm{O} \\
(\% o, \mathrm{PDB})\end{array}$ & $\begin{array}{l}\mathrm{Sr} \\
(\mathrm{ppm})\end{array}$ & $\begin{array}{l}\mathrm{Mn} \\
(\mathrm{ppm})\end{array}$ & $\begin{array}{l}\mathrm{Mn} / \\
\mathrm{Sr}\end{array}$ & $\begin{array}{ll}\text { TOC } & \delta^{13} \mathrm{C}_{\mathrm{TOC}} \\
(\mathrm{mg} / \mathrm{g}) & (\% \circ, \mathrm{PDB})\end{array}$ & $\begin{array}{l}\text { Stratigraphic } \\
\text { units }\end{array}$ \\
\hline \multicolumn{10}{|l|}{ k97.5 } \\
\hline k97.5.10.0 & 10.0 & silt dol (det) & 4.01 & -13.32 & 92.44 & 1207.88 & 13.07 & & $\begin{array}{l}\text { Blaini } \\
\text { Formation }\end{array}$ \\
\hline k97.5.11.0 & 11.0 & silt dol (det) & -3.11 & -12.96 & & & & & \\
\hline k97.5.141.3 & 141.3 & silt dol & 3.26 & -11.75 & & & & & Surface 2 \\
\hline k97.5.143.9 & 143.9 & mud dol & 2.66 & -7.41 & & & & & $\begin{array}{l}\text { Infra Krol } \\
\text { Formation }\end{array}$ \\
\hline k97.5.158.2 & 158.2 & mud $1 \mathrm{~m}$ & 0.4 & -7.39 & 95.46 & 869.91 & 9.11 & & \\
\hline k97.5.163.2 & 163.2 & lm mudst & 1.25 & -5.75 & & & & & \\
\hline k97.5.168.0 & 168.0 & lm mudst & 2.54 & -5.78 & 124.47 & 1078.75 & 8.67 & & \\
\hline k97.5.175.2 & 175.2 & lm mudst & 0.89 & -12.15 & 191.65 & 570.81 & 2.98 & & \\
\hline k97.5.182.0 & 182.0 & lm mudst & 1.7 & -12.27 & & & & & \\
\hline k97.5.187.0 & 187.0 & dol wackest & 2.6 & -12.52 & & & & & \\
\hline k97.5.193.9 & 193.9 & pel grainst & 1.99 & -12.72 & 208.78 & 809.48 & 3.88 & & \\
\hline k97.5.200.4 & 200.4 & dol wackest & 2.24 & -12.05 & 362.33 & 796.07 & 2.2 & & \\
\hline k97.5.205.4 & 205.4 & intra grainst & -2.84 & -9.89 & 130.04 & 737.94 & 5.67 & & \\
\hline k97.5.215.4 & 215.4 & lm mudst & 2.07 & -11.54 & & & & & \\
\hline k97.5.225.4 & 225.4 & pel wackest & 3 & -9.88 & 43.77 & 658.63 & 15.05 & & \\
\hline k97.5.232.5 & 232.5 & pel grainst & 2.77 & -12.74 & & & & & \\
\hline k97.5.266.7 & 266.7 & lm mudst & 3.22 & -10.05 & & & & & Krol A \\
\hline k97.5.276.7 & 276.7 & dol wackest & 4.4 & -10.73 & & & & & \\
\hline k97.5.284.6 & 284.6 & lm mudst & 3.4 & -8.39 & 86.03 & 508.11 & 5.91 & & \\
\hline k97.5.298.4 & 298.4 & pel packst & 2.89 & -10.97 & 509.25 & 887.44 & 1.74 & & \\
\hline k97.5.313.9 & 313.9 & pel wackest & 3.62 & -11.96 & & & & & \\
\hline k97.5.319.9 & 319.9 & intra grainst & 4.36 & -11.84 & 1142.12 & 440.25 & 0.39 & & \\
\hline k97.5.327.7 & 327.7 & $\operatorname{lm}$ mudst & 3.85 & -9.86 & 46.48 & 429.39 & 9.24 & & \\
\hline k97.5.336.3 & 336.3 & dol packst & 3.76 & -11.3 & 680.54 & 701.2 & 1.03 & & \\
\hline k97.5.339.9 & 339.9 & dol packst & -7.59 & -6.02 & & & & & \\
\hline k97.5.344.6 & 344.6 & dol packst & 3.21 & -12.07 & 877.29 & 571.17 & 0.65 & & \\
\hline k97.5.381.4 & 381.4 & silt/ch dol & -8.44 & -7.9 & 98.37 & 831.4 & 8.45 & & Krol B \\
\hline k97.5.382.0 & 382.0 & silt dol & -8.72 & -8.25 & 122.78 & 3214.99 & 26.18 & & \\
\hline k97.5.388.0 & 388.0 & silt dol & -9.04 & -14.11 & 308.74 & 699.77 & 2.27 & & \\
\hline k97.5.388.5 & 388.5 & silt dol & -7.93 & -13.24 & 321.31 & 683.63 & 2.13 & & \\
\hline k97.5.389.9 & 389.9 & silt dol & -7.21 & -6.17 & 74.48 & 1285.05 & 17.25 & & \\
\hline k97.5.391.3 & 391.3 & silt dol & -7.85 & -13.09 & 189.42 & 701.05 & 3.7 & & \\
\hline k97.5.391.7 & 391.7 & silt dol & -8.29 & -13.28 & & & & & \\
\hline k97.5.391.9 & 391.9 & silt dol & -8.11 & -13.05 & 218.32 & 419.59 & 1.92 & & \\
\hline k97.5.392.8 & 392.8 & silt dol & -8.54 & -13.42 & 285.58 & 902.08 & 3.16 & & \\
\hline k97.5.393.8 & 393.8 & silt dol & -8.61 & -13.24 & & & & & Surface 3 \\
\hline k97.5.399.1 & 399.1 & intra grainst & -8.46 & -13.6 & 201.78 & 778.67 & 3.86 & & \\
\hline k97.5.407.3 & 407.3 & dol grainst & -7.86 & -12.71 & 242.05 & 1036.88 & 4.28 & & \\
\hline k97.5.411.4 & 411.4 & pel packst & -7.42 & -13.27 & & & & & \\
\hline k97.5.421.8 & 421.8 & intra grainst & -8.3 & -13.37 & & & & & \\
\hline k97.5.432.9 & 432.9 & lm mudst & -0.3 & -7.29 & & & & & Krol C \\
\hline k97.5.434.1 & 434.1 & pel packst & 0.49 & -6.79 & 26.1 & 392.2 & 15.02 & & \\
\hline k97.5.436.8 & 436.8 & pel packst & 1.97 & -8.04 & 184.92 & 180.27 & 0.98 & & \\
\hline k97.5.439.5 & 439.5 & dol packst & 0.52 & -11.65 & 157.17 & 115.78 & 0.74 & & \\
\hline k97.5.439.6 & 439.6 & dol wackest & -2.15 & -12.76 & 64.37 & 554.35 & 8.61 & & \\
\hline k97.5.449.8 & 449.8 & vug/ch dol & -0.09 & -12.41 & 95.26 & 462.46 & 4.86 & & \\
\hline k97.5.469.8 & 469.8 & vug/ch dol & 1.11 & -12.47 & 44.9 & 654.57 & 14.58 & & \\
\hline k97.5.490.2 & 490.2 & karstic br & 0.71 & -11.42 & 73.13 & 199.24 & 2.73 & & Surface 4 \\
\hline k97.5.500.2 & 500.2 & karstic br & 0.91 & -12.89 & 59.23 & 569.2 & 9.61 & & \\
\hline k97.5.510.2 & 510.2 & karstic br & 1.5 & -12.17 & 36.49 & 620.5 & 17.01 & & \\
\hline k97.5.513.0 & 513.0 & intra grainst & 6.04 & 3.42 & & & & & \\
\hline k97.5.520.0 & 520.0 & oo grainst & 1.33 & -3.63 & 81.9 & 98.44 & 1.2 & & \\
\hline k97.5.525.0 & 525.0 & oo grainst & 0.76 & -4.25 & & & & & \\
\hline k97.5.547.0 & 547.0 & oo grainst & -2.61 & -1.77 & 91.82 & 898.17 & 9.78 & & Krol C \\
\hline
\end{tabular}


Table 1 (Continued)

\begin{tabular}{|c|c|c|c|c|c|c|c|c|c|c|}
\hline $\begin{array}{l}\text { Sample } \\
\text { number }\end{array}$ & $\begin{array}{l}\text { Position } \\
\text { (m) }\end{array}$ & Lithology* & $\begin{array}{l}\delta^{13} \mathrm{C} \\
(\% o, \mathrm{PDB})\end{array}$ & $\begin{array}{l}\delta^{18} \mathrm{O} \\
(\% o, \mathrm{PDB})\end{array}$ & $\begin{array}{l}\mathrm{Sr} \\
(\mathrm{ppm})\end{array}$ & $\begin{array}{l}\mathrm{Mn} \\
(\mathrm{ppm})\end{array}$ & $\begin{array}{l}\mathrm{Mn} / \\
\mathrm{Sr}\end{array}$ & $\begin{array}{l}\text { TOC } \\
(\mathrm{mg} / \mathrm{g})\end{array}$ & $\begin{array}{l}\delta^{13} \mathrm{C}_{\mathrm{TOC}} \\
(\% \circ, \mathrm{PDB})\end{array}$ & $\begin{array}{l}\text { Stratigraphic } \\
\text { units }\end{array}$ \\
\hline k97.5.565.0 & 565.0 & lm mudst & 4.86 & -7.93 & & & & & & \\
\hline k97.5.585.0 & 585.0 & pel packst & 3.64 & -8.95 & 99.81 & 51.75 & 0.52 & & & \\
\hline k97.5.595.0 & 595.0 & pel packst & 5.78 & -10.4 & & & & & & \\
\hline k97.5.619.5 & 619.5 & ch dol & 1.12 & -4.18 & & & & & & \\
\hline k97.5.634.7 & 634.7 & ch dol & 3.93 & -10.41 & 221.61 & 64.57 & 0.29 & & & \\
\hline k97.5.647.2 & 647.2 & dol packst & 6.62 & -2.32 & 92.92 & 19.04 & 0.21 & & & \\
\hline k97.5.655.0 & 655.0 & mic dol & 4.04 & -4.25 & 110.69 & 80.68 & 0.73 & & & \\
\hline $\mathrm{k} 97.5 .676 .2$ & 676.2 & silt/ch dol & 4.44 & -3.99 & & & & & & Surface 5 \\
\hline k97.5.689.2 & 689.2 & ch dol & 4.64 & -2.24 & 82.01 & 131.11 & 1.6 & & & \\
\hline $\mathrm{k} 97.5 .702 .1$ & 702.1 & ch dol & 2.97 & -2.04 & 167.56 & 348.2 & 2.08 & & & \\
\hline k97.5.722.4 & 722.4 & ch dol & 2.38 & -0.89 & 97.72 & 142.36 & 1.46 & & & \\
\hline k97.5.733.4 & 733.4 & ch dol & 1.69 & -1.17 & 115.38 & 174.85 & 1.52 & & & \\
\hline $\mathrm{k} 97.5 .735 .6$ & 735.6 & ch dol & 1.39 & -5.71 & 372.88 & 530.99 & 1.42 & & & \\
\hline k97.5.742.6 & 742.6 & mud $1 \mathrm{~m}$ & 0.14 & -4.53 & & & & & & \\
\hline k97.5.756.1 & 756.1 & dol packst & 2.24 & -0.04 & 106.41 & 228.2 & 2.15 & & & Krol D \\
\hline $\mathrm{k} 97.5 .810 .0$ & 810.0 & ch dol & -1.08 & -2.08 & 106.11 & 181.11 & 1.71 & & & \\
\hline k97.5.843.9 & 843.9 & mud $1 \mathrm{~m}$ & -12.88 & -7 & 36.63 & 216.19 & 5.9 & & & \\
\hline k97.5.862.7 & 862.7 & $\mathrm{ch} / \mathrm{silt}$ dol & 2.74 & -2.04 & 113.49 & 1883.25 & 16.6 & & & \\
\hline $\mathrm{k} 97.5 .869 .0$ & 869.0 & $\mathrm{ch} / \mathrm{silt}$ dol & -2.26 & -6.17 & 39.16 & 683.83 & 17.46 & & & \\
\hline k97.5.873.6 & 873.6 & $\mathrm{ch} / \mathrm{silt}$ dol & 1.52 & -2.58 & 96.35 & 1203.91 & 12.5 & & & \\
\hline k97.5.878.0 & 878.0 & $\mathrm{ch} / \mathrm{silt}$ dol & -1.65 & -5.03 & 46.73 & 916.25 & 19.61 & & & \\
\hline k97.5.878.4 & 878.4 & $\mathrm{ch} / \mathrm{silt}$ dol & -2.02 & -4.05 & 89.12 & 983.83 & 11.04 & & & \\
\hline k97.5.899.9 & 899.9 & $\mathrm{ch} / \mathrm{silt}$ dol & -0.42 & -2.97 & & & & & & \\
\hline k97.5.922.2 & 922.2 & $\mathrm{ch} / \mathrm{silt}$ dol & -1.49 & -2.41 & & & & & & \\
\hline k97.5.924.7 & 924.7 & $\mathrm{ch} / \mathrm{silt}$ dol & -2.85 & -3.15 & & & & & & Surface 6 \\
\hline k97.5.971.7 & 971.7 & mass/ch dol & -1.45 & -5.46 & 73.81 & 1005.76 & 13.63 & & & \\
\hline k97.5.991.7 & 991.7 & mass/ch dol & 2.87 & -1.19 & 66.11 & 213.91 & 3.24 & & & Krol D \\
\hline k97.5.1011.7 & 1011.7 & mass/ch dol & 1.46 & -0.94 & 50.31 & 112.49 & 2.24 & & & \\
\hline k97.5.1031.7 & 1031.7 & mass/ch dol & 2.94 & & 32.83 & 149.2 & 4.55 & & & \\
\hline k97.5.1051.7 & 1051.7 & mass/ch dol & 2.27 & -1.79 & 41.68 & 96.33 & 2.31 & & & \\
\hline k97.5.1071.7 & 1071.7 & mass/ch dol & 1.64 & -4.09 & 49.63 & 191.24 & 3.85 & & & \\
\hline k97.5.1091.7 & 1091.7 & mass/ch dol & 1.67 & -3.45 & 46.09 & 190.1 & 4.12 & & & Surface 7 \\
\hline \multicolumn{11}{|l|}{ Section $\mathrm{k}$} \\
\hline $\mathrm{k}-1$ & 40.0 & mud dol & -6.72 & -4.73 & 193.95 & 1459.64 & 7.53 & & & Krol E \\
\hline $\mathrm{k}-2$ & 47.0 & mud dol & -5.72 & -5.15 & & & & & & \\
\hline $\mathrm{k}-3$ & 53.5 & mud dol & 0.58 & -5.86 & 47.88 & 188.64 & 3.94 & & & \\
\hline $\mathrm{k}-5$ & 71.5 & mud dol & -8.67 & -4.87 & 214.87 & 588.67 & 2.74 & & & Surface 8 \\
\hline $\mathrm{k}-6$ & 86.0 & mud $1 \mathrm{~lm}$ & -8.84 & -4.2 & & & & & & \\
\hline $\mathrm{k}-7$ & 96.0 & mud $1 \mathrm{~m}$ & -11.05 & -4.25 & 230.05 & 599.91 & 2.61 & & & Tal Group \\
\hline $\mathrm{k}-8$ & 109.0 & mud $1 \mathrm{~m}$ & -12.71 & -5.48 & & & & & & \\
\hline k-9 & 121.0 & bk ch & -12.21 & -4.4 & & & & & & \\
\hline \multicolumn{11}{|c|}{ 3. Samples from Mussoorie syncline } \\
\hline $\mathrm{k} 97.6 .29 .5$ & 29.5 & mud dol & -2.75 & -8.47 & 68.42 & 1035.3 & 15.13 & & & Surface 1 \\
\hline $\mathrm{k} 97.6 .30 .2$ & 30.2 & mud dol & -2.73 & -8.89 & 67.04 & 879.03 & 13.11 & & & \\
\hline k97.6.30.7 & 30.7 & mud dol & -2.69 & & 89.43 & 921.73 & 10.31 & & & \\
\hline k97.6.31.4 & 31.4 & mud dol & -3 & & 51.65 & 922.52 & 17.86 & & & \\
\hline k97.6.32.5 & 32.5 & mud dol & -2.98 & & 66.81 & 1017.12 & 15.22 & & & \\
\hline k97.6.40.3 & 40.3 & mud dol & -2.87 & -6.2 & & & & & & \\
\hline $\mathrm{k} 97.6 .40 .8$ & 40.8 & mud dol & -2.65 & -7.83 & 124.94 & 750.29 & 6.01 & & & $\begin{array}{l}\text { Blaini } \\
\text { Formation }\end{array}$ \\
\hline k97.6.41.3 & 41.3 & mud dol & -2.63 & & 84.3 & 1073.11 & 12.73 & & & \\
\hline k97.6.41.8 & 41.8 & mud dol & -3.23 & -8.26 & 70.52 & 1141.98 & 16.19 & & & \\
\hline k97.6.42.8 & 42.8 & mud dol & -3.02 & & 73.07 & 1016.74 & 13.92 & & & \\
\hline k97.6.43.3 & 43.3 & mud dol & -3.06 & & & & & & & \\
\hline k97.6.43.9 & 43.9 & mud dol & -2.98 & -9.52 & & & & & & \\
\hline
\end{tabular}


Table 1 (Continued)

\begin{tabular}{|c|c|c|c|c|c|c|c|c|c|c|}
\hline $\begin{array}{l}\text { Sample } \\
\text { number }\end{array}$ & $\begin{array}{l}\text { Position } \\
\text { (m) }\end{array}$ & Lithology ${ }^{*}$ & $\begin{array}{l}\delta^{13} \mathrm{C} \\
(\% o, \mathrm{PDB})\end{array}$ & $\begin{array}{l}\delta^{18} \mathrm{O} \\
(\% \circ, \mathrm{PDB})\end{array}$ & $\begin{array}{l}\mathrm{Sr} \\
(\mathrm{ppm})\end{array}$ & $\begin{array}{l}\mathrm{Mn} \\
(\mathrm{ppm})\end{array}$ & $\begin{array}{l}\mathrm{Mn} / \\
\mathrm{Sr}\end{array}$ & $\begin{array}{l}\text { TOC } \\
(\mathrm{mg} / \mathrm{g})\end{array}$ & $\begin{array}{l}\delta^{13} \mathrm{C}_{\mathrm{TOC}} \\
(\% o, \mathrm{PDB})\end{array}$ & $\begin{array}{l}\text { Stratigraphic } \\
\text { units }\end{array}$ \\
\hline k97.6.44.5 & 44.5 & mud dol & -3.38 & -8.52 & & & & & & \\
\hline k97.6.45.1 & 45.1 & mud dol & -3.36 & -8.08 & & & & & & \\
\hline k97.6.45.6 & 45.6 & mud dol & -3.21 & & & & & & & \\
\hline k97.6.46.2 & 46.2 & mud dol & -3.45 & -9.78 & & & & & & \\
\hline k97.6.46.9 & 46.9 & mud dol & -3.54 & & 98.45 & 1107.8 & 11.25 & & & \\
\hline k97.6.47.5 & 47.5 & mud dol & -3.67 & -8.56 & 70.76 & 1009.34 & 14.26 & & & \\
\hline k97.6.48.2 & 48.2 & mud dol & -3.41 & -9.08 & & & & & & \\
\hline k97.6.48.6 & 48.6 & mud dol & -3.12 & -8.75 & & & & & & \\
\hline k97.7 & & & & & & & & & & Surface 1 \\
\hline k97.7.50.1 & 50.1 & mud dol & -3.2 & & 89.23 & 1200.71 & 13.46 & & & \\
\hline k97.7.59.0 & 59.0 & mud dol & -2.98 & -9.41 & & & & & & \\
\hline k97.7.171.6 & 171.6 & silt dol & -2.67 & -10.94 & & & & & & Surface 2 \\
\hline k97.7.173.2 & 173.2 & silt dol & 1.87 & -8.2 & & & & & & \\
\hline k97.7.175.3 & 175.3 & silt dol & 2.52 & -5.72 & 159.4 & 1492.52 & 9.36 & & & \\
\hline k97.7.177.8 & 177.8 & silt dol & 1.94 & & 166.17 & 1456.56 & 8.77 & & & $\begin{array}{l}\text { Infra Krol } \\
\text { Formation }\end{array}$ \\
\hline k97.7.182.6 & 182.6 & silt dol & 0.9 & -9.16 & & & & & & \\
\hline k97.7.189.2 & 189.2 & silt dol & -4.06 & -13.28 & 3182.06 & 150.08 & 0.05 & & & \\
\hline k97.7.267.2 & 267.2 & mud $1 \mathrm{~m}$ & 2.24 & -12.69 & & & & & & \\
\hline k97.7.271.5 & 271.5 & lm mudst & 2.39 & -12.88 & 139.29 & 471.24 & 3.38 & & & \\
\hline k97.7.276.5 & 276.5 & mud $1 \mathrm{~m}$ & 1.25 & -10.26 & 47.28 & 393.08 & 8.31 & & & \\
\hline k97.7.285.1 & 285.1 & lm mudst & 1.59 & -5.76 & 18.31 & 575.92 & 31.5 & & & Krol A \\
\hline k97.7.298.0 & 298.0 & $\begin{array}{l}\text { Microbial } \\
\text { limestone }\end{array}$ & 3.23 & -12.29 & 289.29 & 540.46 & 1.87 & & & \\
\hline k97.7.309.0 & 309.0 & lm mudst & 2.12 & -11.65 & 45.37 & 278.61 & 6.14 & & & \\
\hline k97.7.318.0 & 318.0 & oo packst & 1.41 & -9.92 & 238.96 & 272.36 & 1.14 & & & \\
\hline k97.7.324.3 & 324.3 & ch dol & 0.67 & -12.46 & & & & & & \\
\hline k97.7.327.4 & 327.4 & oo packst & 1.2 & -6.25 & 205.94 & 523.39 & 2.54 & & & \\
\hline k97.7.341.0 & 341.0 & ch dol & -4.97 & -8.14 & & 264.36 & & & & Krol B \\
\hline k97.7.352.0 & 352.0 & ch dol & 2.37 & & 202.25 & 2656.08 & 13.13 & & & \\
\hline k97.7.355.7 & 355.7 & $\mathrm{ch} / \mathrm{silt}$ dol & -7.31 & -5.4 & & & & & & \\
\hline k97.7.356.9 & 356.9 & $\mathrm{ch} / \mathrm{silt}$ dol & -0.06 & -4.95 & 17.91 & 1064.84 & 59.45 & & & Surface 3 \\
\hline k97.7.357.5 & 357.5 & mud lm & 0.13 & & 22.96 & 650.61 & 28.34 & & & \\
\hline k97.7.360.2 & 360.2 & mud $1 \mathrm{~m}$ & -0.89 & -5.71 & & & & & & krol C \\
\hline k97.7.364.2 & 364.2 & mud $1 \mathrm{~m}$ & 1.68 & & & & & & & \\
\hline k97.7.366.5 & 366.5 & mud $1 \mathrm{~m}$ & -0.86 & -6.02 & & & & & & \\
\hline k97.7.370.0 & 370.0 & dol packst & -1.2 & -5.2 & & & & & & \\
\hline k97.7.379.0 & 379.0 & ch dol & -0.28 & & & & & & & Surface 4 \\
\hline k97.7.381.0 & 381.0 & karstic br & 1.57 & -7.59 & 95.45 & 474.62 & 4.97 & & & \\
\hline k97.7.390.0 & 390.0 & karstic br & 1.27 & -10.34 & 75.51 & 409.18 & 5.42 & & & \\
\hline k97.7.396.5 & 396.5 & karstic br & -0.01 & -10.55 & & & & & & \\
\hline k97.7.403.0 & 403.0 & karstic br & -0.52 & & & & & & & \\
\hline k97.7.411.6 & 411.6 & oo grainst & 1.21 & & & & & & & \\
\hline k97.7.417.9 & 417.9 & oo grainst & 0.9 & -6.61 & 71.19 & 432.45 & 6.08 & & & \\
\hline k97.7.420.4 & 420.4 & oo grainst & 1.4 & & 67.79 & 160.23 & 2.36 & & & \\
\hline k97.7.436.4 & 436.4 & oo grainst & 1.4 & -7.54 & & & & & & \\
\hline k97.7.441.2 & 441.2 & oo packst & 1.39 & -6.07 & 53.75 & 292.14 & 5.43 & & & \\
\hline k97.7.444.0 & 444.0 & oo packst & 0.59 & & 55.91 & 345.89 & 6.19 & & & \\
\hline k97.7.451.5 & 451.5 & oo grainst & 1.68 & -8.42 & 59.62 & 545.38 & 9.15 & & & \\
\hline k97.7.462.0 & 462.0 & oo grainst & 1.5 & -7.12 & & & & & & \\
\hline k97.7.465.0 & 465.0 & $\mathrm{mic} / \mathrm{ch}$ dol & 1.18 & -6.79 & 68.1 & 703.82 & 10.34 & & & Krol C \\
\hline k97.7.473.0 & 473.0 & mud lm & -2.19 & -2.03 & 93.85 & 542.13 & 5.78 & & & \\
\hline k97.7.481.0 & 481.0 & $\begin{array}{l}\text { Microbial } \\
\text { wackest }\end{array}$ & 2.13 & -2.46 & 107.9 & 263.7 & 2.44 & & & \\
\hline k97.7.484.0 & 484.0 & $\begin{array}{l}\text { Microbial } \\
\text { wackest }\end{array}$ & -1.33 & -7.16 & & & & & & \\
\hline
\end{tabular}


Table 1 (Continued)

\begin{tabular}{|c|c|c|c|c|c|c|c|c|c|c|}
\hline $\begin{array}{l}\text { Sample } \\
\text { number }\end{array}$ & $\begin{array}{l}\text { Position } \\
\text { (m) }\end{array}$ & Lithology ${ }^{*}$ & $\begin{array}{l}\delta^{13} \mathrm{C} \\
(\% o, \mathrm{PDB})\end{array}$ & $\begin{array}{l}\delta^{18} \mathrm{O} \\
(\% \circ, \mathrm{PDB})\end{array}$ & $\begin{array}{l}\mathrm{Sr} \\
(\mathrm{ppm})\end{array}$ & $\begin{array}{l}\mathrm{Mn} \\
(\mathrm{ppm})\end{array}$ & $\begin{array}{l}\mathrm{Mn} / \\
\mathrm{Sr}\end{array}$ & $\begin{array}{l}\text { TOC } \\
(\mathrm{mg} / \mathrm{g})\end{array}$ & $\begin{array}{l}\delta^{13} \mathrm{C}_{\mathrm{TOC}} \\
(\% o, \mathrm{PDB})\end{array}$ & $\begin{array}{l}\text { Stratigraphic } \\
\text { units }\end{array}$ \\
\hline k97.7.492.1 & 492.1 & pel packst & -2.38 & -3.19 & & & & & & \\
\hline k97.7.502.4 & 502.4 & $\mathrm{mic} / \mathrm{ch} \mathrm{dol}$ & -0.95 & -4.96 & & & & & & \\
\hline k97.7.505.4 & 505.4 & $\mathrm{mic} / \mathrm{ch}$ dol & -1.5 & -6.22 & & & & & & \\
\hline k97.7.508.8 & 508.8 & $\mathrm{mic} / \mathrm{ch}$ dol & -6.97 & -3.24 & 21.79 & 1323.86 & 60.76 & & & \\
\hline k97.7.510.6 & 510.6 & mud $1 \mathrm{~m}$ & -5.4 & -4.09 & 112.7 & 2099.66 & 18.63 & & & \\
\hline k97.7.512.9 & 512.9 & pel wackest & -4.92 & -2.93 & 80.3 & 1258.22 & 15.67 & & & \\
\hline k97.7.519.3 & 519.3 & $\mathrm{mic} / \mathrm{ch}$ dol & 0.26 & & & & & & & \\
\hline k97.7.526.3 & 526.3 & $\mathrm{mic} / \mathrm{ch}$ dol & 3.64 & -2.74 & 92.04 & 217.89 & 2.37 & & & \\
\hline k97.7.532.8 & 532.8 & $\mathrm{mic} / \mathrm{ch}$ dol & -1.69 & -2.22 & & & & & & \\
\hline k97.7.533.0 & 533.0 & mic dol & -1.28 & & & & & & & \\
\hline k97.7.535.0 & 535.0 & ch dol & -0.75 & -2.53 & & & & & & \\
\hline k97.7.537.0 & 537.0 & mic dol & -1.13 & -3.9 & 83.99 & 437.41 & 5.21 & & & \\
\hline k97.7.537.5 & 537.5 & mic dol & -1.88 & -7.65 & 18.82 & 263.15 & 13.98 & & & \\
\hline k97.7.539.0 & 539.0 & $\operatorname{lm}$ mudst & 4.67 & & 107.01 & 732.67 & 6.85 & & & \\
\hline k97.7.546.1 & 546.1 & mic dol & 5.76 & -4.68 & & & & & & \\
\hline k97.7.555.7 & 555.7 & ch dol & 6.7 & -3.76 & 165.46 & 126.11 & 0.76 & & & \\
\hline k97.7.559.7 & 559.7 & mic dol & 5.93 & -3.02 & 98.2 & 51.85 & 0.53 & & & Krol C \\
\hline k97.7.568.0 & 568.0 & ch dol & 3.7 & -3.08 & 105.01 & 444.26 & 4.23 & & & \\
\hline k97.7.576.2 & 576.2 & mic dol & 6.37 & -2.86 & 88.93 & 49.98 & 0.56 & & & \\
\hline k97.7.589.2 & 589.2 & $\mathrm{mic} / \mathrm{ch}$ dol & 3.37 & -2.22 & & & & & & \\
\hline k97.7.599.2 & 599.2 & mic dol & 4.48 & -4.75 & 108.18 & 233.23 & 2.16 & & & \\
\hline k97.7.610.4 & 610.4 & $\mathrm{mic} / \mathrm{ch}$ dol & 5.02 & -5.85 & & & & & & \\
\hline k97.7.616.0 & 616.0 & $\mathrm{mic} / \mathrm{ch}$ dol & 5.67 & & 80.59 & 628.45 & 7.8 & & & \\
\hline k97.7.622.4 & 622.4 & mic dol & 2.98 & -2.14 & & & & & & \\
\hline k97.7.630.4 & 630.4 & $\mathrm{mic} / \mathrm{ch}$ dol & 3.31 & -4.98 & & & & & & \\
\hline k97.7.636.8 & 636.8 & $\mathrm{mic} / \mathrm{ch}$ dol & 3.03 & -2.57 & 157.73 & 202.03 & 1.28 & & & \\
\hline k97.7.637.5 & 637.5 & oo grainst & 1.54 & -13.75 & 51.02 & 607.83 & 11.91 & & & \\
\hline k97.7.645.2 & 645.2 & oo grainst & 1.42 & -6.46 & & & & & & \\
\hline k97.7.653.2 & 653.2 & oo grainst & -0.8 & & & & & & & \\
\hline k97.7.653.4 & 653.4 & $1 \mathrm{~m}$ mudst & -2.92 & -3.33 & 65.57 & 182.47 & 2.78 & & & \\
\hline k97.7.658.2 & 658.2 & $\mathrm{mic} / \mathrm{ch}$ dol & 2.12 & -3.82 & 58.33 & 177.77 & 3.05 & & & \\
\hline k97.7.660.5 & 660.5 & ch dol & -4.53 & -4.75 & & & & & & \\
\hline k97.7.666.3 & 666.3 & oo grainst & 1.63 & -8.21 & & & & & & \\
\hline k97.7.671.1 & 671.1 & oo grainst & 1.29 & -8.58 & & & & & & \\
\hline k97.7.678.1 & 678.1 & oo grainst & 1.75 & & & & & & & \\
\hline k97.7.683.2 & 683.2 & oo grainst & 1.44 & & & & & & & \\
\hline k97.7.689.2 & 689.2 & silt/ch dol & 1.37 & & 40.95 & 151.17 & 3.7 & & & \\
\hline k97.7.697.8 & 697.8 & silt/ch dol & 1.32 & & 47.66 & 100.67 & 2.11 & & & \\
\hline k97.7.699.5 & 699.5 & silt/ch dol & -1.87 & -5.98 & & & & & & Surface 5 \\
\hline k97.7.706.7 & 706.7 & lm mudst & 1.22 & -5.85 & & & & & & \\
\hline k97.7.706.9 & 706.9 & ch dol & -3.85 & & & & & & & \\
\hline k97.7.708.9 & 708.9 & $\operatorname{lm}$ mudst & 2.03 & -3.38 & & & & & & \\
\hline k97.7.711.8 & 711.8 & ch dol & -0.45 & & & & & & & \\
\hline k97.7.716.2 & 716.2 & lm mudst & 3.45 & -1.55 & 84.07 & 190.01 & 2.26 & & & \\
\hline k97.7.716.8 & 716.8 & pisolite & -2.06 & -2.39 & 46.66 & 500.07 & 10.72 & & & \\
\hline k97.7.724.3 & 724.3 & ch dol & 0.4 & & & & & & & \\
\hline k97.7.725.7 & 725.7 & dol packst & -0.12 & -12.08 & & & & & & Krol D \\
\hline k97.7.729.7 & 729.7 & mic dol & 3.6 & -5.49 & & & & & & \\
\hline k97.7.730.2 & 730.2 & dol packst & 4.74 & -2.37 & & & & & & \\
\hline k97.7.739.2 & 739.2 & mic dol & 4.04 & -3.57 & & & & & & \\
\hline k97.7.741.0 & 741.0 & mic packst & 5.89 & -3.17 & & & & & & \\
\hline k97.7.752.0 & 752.0 & mic dol & 6.24 & -5.33 & & & & & & \\
\hline k97.7.756.0 & 756.0 & mic dol & 7.1 & -3.5 & 56.93 & 291.85 & 5.13 & & & \\
\hline k97.7.764.1 & 764.1 & mic dol & 3.69 & -4.29 & & & & & & \\
\hline k97.7.770.2 & 770.2 & mic dol & 5.87 & -1.04 & 78.15 & 91.52 & 1.17 & & & \\
\hline k97.7.774.2 & 774.2 & dol packst & 1.48 & & & & & & & \\
\hline
\end{tabular}


Table 1 (Continued)

\begin{tabular}{|c|c|c|c|c|c|c|c|c|c|c|}
\hline $\begin{array}{l}\text { Sample } \\
\text { number }\end{array}$ & $\begin{array}{l}\text { Position } \\
\text { (m) }\end{array}$ & Lithology & $\begin{array}{l}\delta^{13} \mathrm{C} \\
(\% o, \mathrm{PDB})\end{array}$ & $\begin{array}{l}\delta^{18} \mathrm{O} \\
(\% \circ, \mathrm{PDB})\end{array}$ & $\begin{array}{l}\mathrm{Sr} \\
(\mathrm{ppm})\end{array}$ & $\begin{array}{l}\mathrm{Mn} \\
(\mathrm{ppm})\end{array}$ & $\begin{array}{l}\mathrm{Mn} / \\
\mathrm{Sr}\end{array}$ & $\begin{array}{l}\text { TOC } \\
(\mathrm{mg} / \mathrm{g})\end{array}$ & $\begin{array}{l}\delta^{13} \mathrm{C}_{\mathrm{TOC}} \\
(\% o, \mathrm{PDB})\end{array}$ & $\begin{array}{l}\text { Stratigraphic } \\
\text { units }\end{array}$ \\
\hline \multicolumn{11}{|l|}{ k97.8 } \\
\hline $\mathrm{k} 97.8 .8 .0$ & 8.0 & $\mathrm{mic} / \mathrm{ch}$ dol & 5.4 & -2.5 & & & & & & \\
\hline k97.8.14.6 & 14.6 & pel packst & 4.47 & -3.9 & & & & & & \\
\hline k97.8.20.8 & 20.8 & mic dol & 5.39 & -3.02 & & & & & & \\
\hline k97.8.22.1 & 22.1 & mic dol & 7.63 & -3.3 & & & & & & \\
\hline k97.8.27.7 & 27.7 & intra packst & 5.91 & -4.6 & & & & & & \\
\hline k97.8.31.7 & 31.7 & ch dol & 2.99 & -3.28 & & & & & & \\
\hline k97.8.32.7 & 32.7 & dol packst & 1.57 & -4.8 & & & & & & \\
\hline k97.8.39.7 & 39.7 & mic dol & 3.39 & -2.1 & 94.14 & 82.84 & 0.88 & & & \\
\hline k97.8.47.1 & 47.1 & mic dol & 4.1 & -3.2 & & & & & & \\
\hline k97.8.53.5 & 53.5 & mic dol & 3.06 & -4.4 & & & & & & \\
\hline k97.8.54.7 & 54.7 & intra packst & 5.77 & -1.99 & 90.46 & 37.15 & 0.41 & & & \\
\hline k97.8.59.9 & 59.9 & mic dol & 5.09 & -2.64 & & & & & & \\
\hline k97.8.66.3 & 66.3 & mic dol & 1.12 & -5.81 & & & & & & Krol C \\
\hline k97.8.72.7 & 72.7 & $\mathrm{mic} / \mathrm{ch}$ dol & 3.47 & -1.76 & & & & & & \\
\hline k97.8.75.9 & 75.9 & ch dol & -1.22 & -4.07 & & & & & & \\
\hline k97.8.79.1 & 79.1 & ch dol & 7.8 & -1.6 & & & & & & \\
\hline k97.8.85.5 & 85.5 & ch dol & 6.5 & -3.14 & & & & & & \\
\hline k97.8.91.9 & 91.9 & ch dol & 6.77 & -1.51 & & & & & & \\
\hline k97.8.98.3 & 98.3 & ch dol & 4.79 & -2.4 & & & & & & \\
\hline k97.8.103.7 & 103.7 & ch dol & 5.11 & -5.88 & & & & & & \\
\hline k97.8.111.2 & 111.2 & intra packst & 5.74 & -5.49 & & & & & & \\
\hline k97.8.113.0 & 113.0 & intra grainst & 8.09 & -7.25 & & & & & & \\
\hline k97.8.120.5 & 120.5 & pel grainst & -1.22 & -11.39 & & & & & & \\
\hline k97.8.128.0 & 128.0 & intra grainst & 6.2 & -5.65 & & & & & & \\
\hline k97.8.131.0 & 131.0 & intra grainst & 1.37 & -6.5 & & & & & & \\
\hline k97.8.138.0 & 138.0 & oo grainst & 6.63 & -3.04 & & & & & & \\
\hline k97.8.145.0 & 145.0 & ch dol & 7.55 & -2.2 & 102.53 & 53.87 & 0.53 & & & \\
\hline k97.8.150.6 & 150.6 & ch dol & 4.68 & -4.75 & 87.75 & 507.34 & 5.78 & & & Surface 5 \\
\hline k97.8.151.2 & 151.2 & mud $1 \mathrm{~m}$ & -2.08 & -1.15 & 105.62 & 30.2 & 0.29 & & & \\
\hline k97.8.153.3 & 153.3 & lm mudst & 2.87 & -2.6 & 93 & 217.37 & 2.34 & & & \\
\hline k97.8.160.5 & 160.5 & ch dol & 3.59 & -3.63 & & & & & & \\
\hline k97.8.175.5 & 175.5 & mic dol & 2.98 & -4.75 & 66.45 & 333.62 & 5.02 & & & \\
\hline k97.8.176.5 & 176.5 & intra packst & 5.81 & -6.01 & 75.6 & 194.4 & 2.57 & & & \\
\hline k97.8.183.5 & 183.5 & mic dol & 5.25 & -3.79 & 98.24 & 113.92 & 1.16 & & & \\
\hline k97.8.190.5 & 190.5 & $\mathrm{mic} / \mathrm{ch}$ dol & 5.86 & -2.79 & 82.61 & 223.78 & 2.71 & & & Krol D \\
\hline k97.8.192.5 & 192.5 & pel packst & 3.04 & -10.15 & 105.44 & 275.1 & 2.61 & & & \\
\hline k97.8.193.0 & 193.0 & mic dol & 1.87 & -10.64 & 118.04 & 393.51 & 3.33 & & & \\
\hline k97.8.195.5 & 195.5 & intra packst & 1.43 & -9.75 & 109.05 & 353.68 & 3.24 & & & \\
\hline k97.8.196.0 & 196.0 & intra packst & 7.04 & -3.25 & 102.53 & 81.74 & 0.8 & & & \\
\hline k97.8.197.0 & 197.0 & pel packst & 5.2 & -5.04 & 138.54 & 498.23 & 3.6 & & & \\
\hline k97.8.200.0 & 200.0 & mic dol & 0.67 & -1.82 & 92.53 & 419.15 & 4.53 & & & \\
\hline k97.8.208.0 & 208.0 & intra packst & 6.61 & -4.06 & & & & & & \\
\hline k97.8.208.5 & 208.5 & intra packst & 6.25 & -4.25 & & & & & & \\
\hline k97.8.210.0 & 210.0 & intra grainst & 6.21 & -2.54 & & & & & & Krol D \\
\hline k97.8.214.0 & 214.0 & intra packst & 4.42 & -5.4 & & & & & & \\
\hline $\mathrm{k} 97.8 .224 .0$ & 224.0 & pel packst & 5.42 & -4.78 & & & & & & \\
\hline k97.8.230.0 & 230.0 & intra packst & 4.99 & -6.96 & & & & & & \\
\hline k97.8.232.0 & 232.0 & intra grainst & 5.26 & -9.37 & & & & & & \\
\hline $\mathrm{k} 97.8 .236 .0$ & 236.0 & intra packst & 7 & -3.56 & & & & & & \\
\hline k97.8.240.0 & 240.0 & intra packst & 2.66 & -0.72 & & & & & & \\
\hline k97.8.246.0 & 246.0 & intra packst & 7.84 & -2.6 & 67.03 & 115.91 & 1.73 & & & \\
\hline k97.8.261.0 & 261.0 & oo grainst & 4.37 & -2.4 & & & & & & \\
\hline k97.8.263.0 & 263.0 & mic dol & 4.41 & -1.8 & & & & & & \\
\hline k97.8.274.5 & 274.5 & mic dol & 4.12 & -3.59 & & & & & & \\
\hline k97.8.284.5 & 284.5 & mic dol & 6.54 & -0.9 & & & & & & \\
\hline k97.8.294.5 & 294.5 & mic dol & 6.94 & -6.32 & 123.34 & 125.69 & 1.02 & & & \\
\hline k97.8.304.5 & 304.5 & mic dol & 5.11 & -4.17 & & & & & & \\
\hline
\end{tabular}


Table 1 (Continued)

\begin{tabular}{|c|c|c|c|c|c|c|c|c|c|c|}
\hline $\begin{array}{l}\text { Sample } \\
\text { number }\end{array}$ & $\begin{array}{l}\text { Position } \\
\text { (m) }\end{array}$ & Lithology* & $\begin{array}{l}\delta^{13} \mathrm{C} \\
(\% o, \mathrm{PDB})\end{array}$ & $\begin{array}{l}\delta^{18} \mathrm{O} \\
(\% \circ, \mathrm{PDB})\end{array}$ & $\begin{array}{l}\mathrm{Sr} \\
(\mathrm{ppm})\end{array}$ & $\begin{array}{l}\mathrm{Mn} \\
(\mathrm{ppm})\end{array}$ & $\begin{array}{l}\mathrm{Mn} / \\
\mathrm{Sr}\end{array}$ & $\begin{array}{l}\text { TOC } \\
(\mathrm{mg} / \mathrm{g})\end{array}$ & $\begin{array}{l}\delta^{13} \mathrm{C}_{\mathrm{TOC}} \\
(\% o, \text { PDB })\end{array}$ & $\begin{array}{l}\text { Stratigraphic } \\
\text { units }\end{array}$ \\
\hline k97.8.314.5 & 314.5 & mic dol & 3.85 & -4.3 & & & & & & \\
\hline k97.8.324.5 & 324.5 & pel wackest & 3.55 & -1.6 & 72.12 & 251.68 & 3.5 & & & \\
\hline k97.8.334.5 & 334.5 & mic dol & 1.62 & -1.09 & 85.8 & 236.22 & 2.75 & & & \\
\hline k97.8.340.5 & 340.5 & ch dol & 6.04 & -3.1 & 98.13 & 265.8 & 2.71 & & & \\
\hline k97.8.350.5 & 350.5 & $\mathrm{mic} / \mathrm{ch}$ dol & 4.78 & -1.38 & 90.28 & 304.3 & 3.37 & & & \\
\hline k97.8.360.5 & 360.5 & ch dol & 4.12 & -0.4 & 107.48 & 212.23 & 1.98 & & & \\
\hline k97.8.370.5 & 370.5 & $\mathrm{mic} / \mathrm{ch}$ dol & 3.04 & -1.71 & & & & & & \\
\hline k97.8.380.5 & 380.5 & mic dol & 4.06 & -0.1 & 98.95 & 96.81 & 0.98 & & & \\
\hline k97.8.390.5 & 390.5 & mic dol & 2.85 & -0.6 & 88.53 & 95.97 & 1.08 & & & \\
\hline k97.8.400.5 & 400.5 & mic dol & 3.3 & -1.44 & & & & & & \\
\hline k97.8.410.5 & 410.5 & mic dol & 2.42 & -0.43 & & & & & & \\
\hline k97.8.411.0 & 411.0 & oo grainst & 2.6 & -0.12 & & & & & & \\
\hline k97.8.421.0 & 421.0 & mic dol & 1.94 & 0.26 & & & & & & \\
\hline k97.8.424.0 & 424.0 & mic dol & 2.36 & -0.6 & & & & & & \\
\hline k97.8.434.0 & 434.0 & mic dol & 2.06 & -1.76 & 106.88 & 561.7 & 5.26 & & & \\
\hline k97.8.444.0 & 444.0 & $\mathrm{mic} / \mathrm{ch}$ dol & 0.87 & -8.16 & & & & & & \\
\hline k97.8.454.0 & 454.0 & ch dol & 2.75 & -2.9 & & & & & & \\
\hline k97.8.464.4 & 464.4 & ch dol & 2.29 & -1.04 & & & & & & \\
\hline k97.8.470.0 & 470.0 & ch dol & 2.31 & 0.2 & & & & & & \\
\hline k97.8.480.0 & 480.0 & ch dol & 1.54 & -2.37 & & & & & & \\
\hline k97.8.490.0 & 490.0 & $\mathrm{mic} / \mathrm{ch}$ dol & 0.44 & -10.35 & & & & & & \\
\hline k97.8.498.0 & 498.0 & ch dol & -1.14 & -10.8 & & & & & & \\
\hline k97.8.508.0 & 508.0 & mic dol & 1.55 & -3.2 & & & & & & \\
\hline k97.8.518.0 & 518.0 & $\mathrm{mic} / \mathrm{ch}$ dol & 2.14 & -1.12 & & & & & & \\
\hline k97.8.528.0 & 528.0 & mass/silt dol & 0.08 & -2.06 & & & & & & Surface 6 \\
\hline k97.8.532.0 & 532.0 & ch dol & 1.31 & -6.5 & & & & & & \\
\hline k97.8.535.0 & 535.0 & intra packst & 2.01 & -1.9 & & & & & & \\
\hline k97.8.539.0 & 539.0 & mud lm & -0.51 & -4.51 & & & & & & \\
\hline k97.8.543.0 & 543.0 & intra packst & -0.9 & -3 & & & & & & \\
\hline k97.8.551.0 & 551.0 & pel wackest & -3.69 & -9.14 & & & & & & \\
\hline k97.8.557.5 & 557.5 & intra packst & -4.94 & -3.33 & & & & & & \\
\hline k97.8.567.5 & 567.5 & intra packst & -0.55 & -2.1 & & & & & & \\
\hline k97.8.573.1 & 573.1 & pel wackest & 0.7 & -8.19 & & & & & & Krol D \\
\hline k97.8.576.1 & 576.1 & intra packst & -4.19 & -10.7 & & & & & & \\
\hline k97.8.578.1 & 578.1 & intra packst & -4.86 & -8.6 & & & & & & \\
\hline k97.8.591.4 & 591.4 & dol wackest & 0.37 & -1.9 & 104.09 & 205.06 & 1.97 & & & \\
\hline k97.8.595.0 & 595.0 & dol packst & -3.11 & -9.9 & & & & & & \\
\hline k97.8.601.0 & 601.0 & intra packst & -1.94 & -9.06 & & & & & & \\
\hline k97.8.611.0 & 611.0 & $\mathrm{mic} / \mathrm{ch}$ dol & 1.42 & -3.11 & & & & & & \\
\hline k97.8.621.0 & 621.0 & mass/ch dol & 2.37 & -1.9 & & & & & & \\
\hline k97.8.631.0 & 631.0 & mass/ch dol & 3.53 & 0.41 & & & & & & \\
\hline k97.8.640.0 & 640.0 & $\mathrm{mic} / \mathrm{ch}$ dol & -0.27 & -3.87 & & & & & & \\
\hline k97.8.642.0 & 642.0 & $\mathrm{mic} / \mathrm{ch}$ dol & -0.43 & -1.25 & & & & & & \\
\hline k97.8.646.0 & 646.0 & dol packst & 0.19 & -3.1 & & & & & & \\
\hline k97.8.656.0 & 656.0 & mass/ch dol & 2.78 & -1.71 & 69.25 & 178.09 & 2.57 & & & \\
\hline k97.8.666.0 & 666.0 & mass/ch dol & 3.71 & 2.9 & & & & & & \\
\hline k97.8.676.0 & 676.0 & mass/ch dol & 4.05 & -3.7 & 37.74 & 95.75 & 2.54 & & & \\
\hline k97.8.686.0 & 686.0 & mass/ch dol & 2.2 & -7.15 & & & & & & \\
\hline k97.8.696.0 & 696.0 & mass/ch dol & 0.98 & -8.11 & & & & & & \\
\hline k97.8.706.0 & 706.0 & mass/ch dol & 0.28 & -7.69 & 42.24 & 116.99 & 2.77 & & & \\
\hline k97.8.716.0 & 716.0 & mass/ch dol & 3.58 & -9.43 & & & & & & \\
\hline k97.8.726.0 & 726.0 & mass/ch dol & 0.8 & -8.98 & & & & & & \\
\hline k97.8.737.0 & 737.0 & mass/ch dol & 0.9 & -5.33 & & & & & & \\
\hline k97.8.745.0 & 745.0 & mass/ch dol & 1.59 & -8.81 & 39.87 & 101.56 & 2.55 & & & \\
\hline k97.8.753.0 & 753.0 & mass/ch dol & 0.22 & -7.6 & & & & & & \\
\hline k97.8.753.5 & 753.5 & mass/ch dol & 0.76 & -7.62 & & & & & & Surface 7 \\
\hline k97.8.754.3 & 754.3 & silt packst & -0.53 & -3.89 & & & & & & \\
\hline k97.8.770.3 & 770.3 & mud dol & 0.27 & -6.68 & & & & & & \\
\hline
\end{tabular}


Table 1 (Continued)

\begin{tabular}{|c|c|c|c|c|c|c|c|c|c|c|}
\hline $\begin{array}{l}\text { Sample } \\
\text { number }\end{array}$ & $\begin{array}{l}\text { Position } \\
\text { (m) }\end{array}$ & Lithology* & $\begin{array}{l}\delta^{13} \mathrm{C} \\
(\% \circ, \mathrm{PDB})\end{array}$ & $\begin{array}{l}\delta^{18} \mathrm{O} \\
(\% \circ, \mathrm{PDB})\end{array}$ & $\begin{array}{l}\mathrm{Sr} \\
(\mathrm{ppm})\end{array}$ & $\begin{array}{l}\mathrm{Mn} \\
(\mathrm{ppm})\end{array}$ & $\begin{array}{l}\mathrm{Mn} / \\
\mathrm{Sr}\end{array}$ & $\begin{array}{l}\text { TOC } \\
(\mathrm{mg} / \mathrm{g})\end{array}$ & $\begin{array}{l}\delta^{13} \mathrm{C}_{\mathrm{TOC}} \\
(\% \circ, \mathrm{PDB})\end{array}$ & $\begin{array}{l}\text { Stratigraphic } \\
\text { units }\end{array}$ \\
\hline k97.8.778.3 & 778.3 & mic dol & -1.64 & -7.82 & 69.78 & 392.8 & 5.63 & & & \\
\hline k97.8.780.0 & 780.0 & silt dol & 0.4 & -8.29 & 50.3 & 75.45 & 1.5 & & & Krol E \\
\hline k97.8.785.0 & 785.0 & mud dol & 0.16 & -4.92 & & & & & & \\
\hline k97.8.789.0 & 789.0 & mud dol & 0.21 & -5.14 & & & & & & \\
\hline k97.8.795.0 & 795.0 & silt dol & 0.06 & -3.2 & & & & & & \\
\hline k97.8.802.0 & 802.0 & silt dol & -0.11 & -2.9 & & & & & & \\
\hline k97.8.803.0 & 803.0 & bk ch & -17.43 & -5.9 & & & & & & Surface 8 \\
\hline \multicolumn{11}{|l|}{ S97.1 } \\
\hline S97.1.35.0 & 35.0 & mud dol & 1.94 & -11.62 & & & & & & $\begin{array}{l}\text { Infra Krol } \\
\text { Formation }\end{array}$ \\
\hline S97.1.35.7 & 35.7 & mud dol & 2.21 & -11.4 & & & & & & \\
\hline S97.1.57.3 & 57.3 & intra dol & 1.93 & -12.3 & & & & & & \\
\hline S97.1.57.8 & 57.8 & intra dol & 3.18 & -1.53 & & & & & & \\
\hline S97.1.58.1 & 58.1 & mud dol & 1.95 & -6.46 & & & & & & \\
\hline \multicolumn{11}{|l|}{ S97.2 } \\
\hline S97.2.65 & 65.0 & intra packst & 3.02 & -13.81 & & & & & & \\
\hline S97.2.83 & 83.0 & intra packst & 3.22 & -6.41 & 470.6 & 323.05 & 0.69 & & & \\
\hline S97.2.91 & 91.0 & lm mudst & 4.19 & -10.8 & & & & & & Krol A \\
\hline S97.2.144 & 144.0 & Wackestone & 2.44 & -5.86 & & & & & & \\
\hline S97.2.170.1 & 170.1 & silt/ch dol & 1.82 & -7.01 & & & & & & Krol B \\
\hline S97.2.211 & 211.0 & silt/ch dol & -0.39 & -6.38 & 123.2 & 1165.63 & 9.46 & & & Surface 3 \\
\hline S97.2.221 & 221.0 & intra packst & -7.99 & -11.71 & 95.99 & & & & & \\
\hline S97.2.230 & 230.0 & mic dol & -7.71 & -11.09 & & & & & & Krol C \\
\hline S97.2.236 & 236.0 & $\mathrm{ch} / \mathrm{mic}$ dol & 0.49 & -10.27 & & & & & & \\
\hline S97.2.240 & 240.0 & mic dol & -0.16 & -7.66 & 43.98 & & & & & \\
\hline S97.2.245 & 245.0 & $\mathrm{mic} / \mathrm{ch}$ dol & -0.31 & -9.86 & & & & & & Surface 4 \\
\hline S97.2.257 & 257.0 & karstic br & -0.16 & -12.45 & & & & & & \\
\hline S97.2.265 & 265.0 & oo grainst & 0.49 & -11.17 & 49.61 & & & & & Krol C \\
\hline S97.2.275 & 275.0 & oo packst & -1.16 & -11.85 & 49.48 & & & & & \\
\hline S97.2.290 & 290.0 & oo grainst & -1.01 & -5.4 & & & & & & \\
\hline \multicolumn{11}{|l|}{ M1 } \\
\hline M1SB-1 & 321.0 & mic dol & 6.04 & -5.42 & & & & & & Krol C \\
\hline M1SB-2 & 334.0 & ch dol & 6.18 & -4.32 & 82.93 & 62.9 & 0.76 & & & \\
\hline M1SB-3 & 342.0 & ch dol & 3.75 & -3.39 & & & & & & Surface 5 \\
\hline M1SB-4 & 345.0 & lm mudst & 3.99 & -3.91 & 81.69 & 227.05 & 2.78 & & & \\
\hline M1SB-5 & 348.5 & mud $1 \mathrm{~m}$ & 3.73 & -3.5 & 100.86 & 183.78 & 1.82 & & & \\
\hline M1SB-6 & 351.0 & ch dol & 3.23 & -3.18 & & & & & & Krol D \\
\hline M1SB-7 & 352.0 & ch dol & 3.1 & -2.48 & & & & & & \\
\hline M1SB-8 & 364.0 & mic dol & 3.64 & -1.61 & & & & & & \\
\hline M1SB-10 & 378.0 & mic dol & 3.07 & -5.19 & 70.68 & & & & & \\
\hline M1SB-11 & 391.0 & mic dol & 3.47 & -6.37 & 108.68 & & & & & \\
\hline \multicolumn{11}{|l|}{ M2 } \\
\hline M2-1 & 377.0 & oo grainst & 6.14 & -5.37 & 44.48 & 453.96 & 10.21 & & & Krol C \\
\hline M2-2 & 384.0 & oo grainst & 1.47 & -6.54 & 49.12 & 530.23 & 10.8 & & & \\
\hline M2-3 & 398.0 & oo grainst & 1.96 & -8.83 & 49.56 & 430.04 & 8.68 & & & \\
\hline M2-4 & 404.0 & ch dol & 0.97 & -8.35 & & & & 0.54 & -27.9 & \\
\hline M2-5 & 422.0 & ch dol & -4.4 & -3.07 & & & & 0.43 & -23.5 & \\
\hline M2-6 & 432.0 & ch dol & -1.73 & -2.99 & & & & & & \\
\hline M2-7 & 438.0 & ch dol & & & & & & 0.21 & -24.5 & Surface 5 \\
\hline M2-8 & 442.0 & lm mudst & -1.75 & -1.78 & & & & 0.16 & -22.3 & \\
\hline M2-10 & 447.0 & ch dol & 3.39 & -3.02 & 82.65 & 234.12 & 2.83 & & & \\
\hline M2-11 & 450.0 & mud $1 \mathrm{~lm}$ & -0.65 & -3.44 & 74.41 & 256.77 & 3.45 & 0.46 & -25.0 & \\
\hline M2-12 & 451.0 & ch dol & 0.01 & -3.89 & 108.15 & 626.48 & 5.8 & 0.91 & -23.8 & Krol D \\
\hline M2-13 & 453.0 & ch dol & 3.56 & -3.36 & & & & 0.5 & -25.5 & \\
\hline M2-14 & 461.0 & mic dol & 5.18 & -5.01 & 145.6 & 184.88 & 1.27 & & & \\
\hline M2-15 & 485.0 & mic dol & 3.82 & -4.51 & 118.2 & 220.5 & 1.87 & & & \\
\hline
\end{tabular}


Table 1 (Continued)

\begin{tabular}{|c|c|c|c|c|c|c|c|c|c|c|}
\hline $\begin{array}{l}\text { Sample } \\
\text { number }\end{array}$ & $\begin{array}{l}\text { Position } \\
(\mathrm{m})\end{array}$ & Lithology* & $\begin{array}{l}\delta^{13} \mathrm{C} \\
(\% o, \mathrm{PDB})\end{array}$ & $\begin{array}{l}\delta^{18} \mathrm{O} \\
(\% \circ, \mathrm{PDB})\end{array}$ & $\begin{array}{l}\mathrm{Sr} \\
(\mathrm{ppm})\end{array}$ & $\begin{array}{l}\mathrm{Mn} \\
(\mathrm{ppm})\end{array}$ & $\begin{array}{l}\mathrm{Mn} / \\
\mathrm{Sr}\end{array}$ & $\begin{array}{l}\text { TOC } \\
(\mathrm{mg} / \mathrm{g})\end{array}$ & $\begin{array}{l}\delta^{13} \mathrm{C}_{\mathrm{TOC}} \\
(\%, \mathrm{PDB})\end{array}$ & $\begin{array}{l}\text { Stratigraphic } \\
\text { units }\end{array}$ \\
\hline \multicolumn{11}{|l|}{ M7 } \\
\hline M7-1 & 5.0 & mass/ch dol & 0.63 & -6.74 & 46.79 & 232.74 & 4.97 & & & \\
\hline M7-3 & 23.0 & mass/ch dol & 1.14 & -5.29 & 49.13 & 98.72 & 2.01 & & & \\
\hline M7-4 & 36.0 & mass/ch dol & 0.23 & -4.58 & 57.12 & 200.84 & 3.52 & & & Krol D \\
\hline M7-5 & 41.5 & mass/ch dol & 3.39 & -1.7 & & & & & & \\
\hline M7-6 & 48.0 & mass/ch dol & 2.77 & -1.95 & & & & & & Surface 7 \\
\hline M7-7 & 51.5 & mud lm & 1.51 & -1.63 & 48.46 & 151.28 & 3.12 & & & \\
\hline M7-8 & 59.0 & silt packst & 1 & -3.36 & & & & & & Krol E \\
\hline M7-9 & 69.0 & mud dol & 0.68 & -3.85 & 28.27 & 247.56 & 8.76 & & & \\
\hline \multicolumn{11}{|l|}{ M8 } \\
\hline M8-1 & 4.0 & mass/ch dol & 0.23 & -11.2 & 246.94 & 313.47 & 1.27 & & & Krol D \\
\hline M8-2 & 23.0 & mass/ch dol & 0.45 & -10.3 & 94.99 & 376.05 & 3.96 & & & \\
\hline M8-3 & 40.5 & mass/ch dol & 0.62 & -10.25 & 42.47 & 283.56 & 6.68 & & & Surface 7 \\
\hline M8-4 & 44.5 & mud lm & 0.47 & -8.08 & 48.46 & 741.05 & 15.3 & & & \\
\hline M8-5 & 54.0 & mud dol & 1.17 & -4.53 & 67.41 & 221.42 & 3.29 & & & \\
\hline M8-6 & 61.5 & mud dol & 1.39 & -6.03 & 58.85 & 362.89 & 6.17 & & & \\
\hline M8-8 & 88.0 & mud dol & -0.45 & -5.1 & 63.92 & 308.83 & 4.83 & & & Krol E \\
\hline M8-9 & 91.0 & $\mathrm{mic} / \mathrm{ch}$ dol & 0.19 & -4.15 & & & & & & \\
\hline M8-10 & 98.5 & $\mathrm{mic} / \mathrm{ch}$ dol & -0.29 & -8.79 & & & & & & \\
\hline M8-12 & 105.0 & silt dol & -2.3 & -9.35 & 47.78 & 763.35 & 15.98 & & & Surface 8 \\
\hline M8-13 & 109.0 & phosph & 0.63 & 5.18 & 164.11 & & & & & \\
\hline M8-14 & 116.0 & phosph & -2.27 & -2.12 & 202.96 & 193.43 & 0.95 & & & Tal Group \\
\hline \multicolumn{11}{|l|}{ M9 } \\
\hline M9-1 & 5.0 & mass/ch dol & & & 121.51 & 446.12 & 3.67 & & & \\
\hline M9-2 & 17.0 & mass/ch dol & 0.5 & -7.59 & 49.2 & 283.73 & 5.77 & & & Krol D \\
\hline M9-3 & 31.0 & mass/ch dol & 1 & -10.03 & & & & & & \\
\hline M9-4 & 41.5 & mass/ch dol & -1.3 & -10.29 & & & & & & Surface 7 \\
\hline M9-5 & 47.0 & mud dol & 0.63 & -9.8 & & & & & & \\
\hline M9-6 & 50.0 & silt packst & 0.48 & -10.24 & 34.57 & 407.31 & 11.78 & & & \\
\hline M9-7 & 59.0 & silt packst & 0.76 & -9.67 & & & & & & Krol E \\
\hline M9-8 & 72.5 & mud dol & 0.65 & -8.43 & & & & & & \\
\hline M9-9 & 81.5 & mud dol & -5.54 & -9.47 & 49.99 & 479.44 & 9.59 & & & \\
\hline M9-10 & 89.0 & mic dol & 0.3 & -9.8 & & & & & & Surface 8 \\
\hline M9-11 & 94.0 & Phosphorite & -6.55 & -8.04 & & & & & & \\
\hline M9-12 & 97.0 & Phosphorite & -8.69 & -6.17 & 1406.21 & 283.07 & 0.2 & & & Tal Group \\
\hline M9-14 & 109.0 & Phosphorite & -9.89 & -13.02 & & & & & & \\
\hline \multicolumn{11}{|l|}{ k98.1 } \\
\hline k98.1.100.5 & 100.5 & lm mudst & 1.9 & -6.72 & & & & & & \\
\hline k98.1.102.0 & 102.0 & lm mudst & 3.02 & -11.32 & & & & & & \\
\hline k98.1.112.0 & 112.0 & mud $1 \mathrm{~m}$ & 3.78 & -12.52 & & & & & & \\
\hline k98.1.123.0 & 123.0 & mud lm & 3.24 & -11.18 & & & & & & \\
\hline k98.1.129.0 & 129.0 & mud $1 \mathrm{~m}$ & 1.87 & -7.6 & & & & & & \\
\hline k98.1.145.0 & 145.0 & lm mudst & 1.82 & -8.73 & & & & & & Krol A \\
\hline k98.1.152.0 & 152.0 & lm mudst & 2.01 & -8.01 & & & & & & \\
\hline k98.1.166.0 & 166.0 & mud lm & 3.01 & -12.03 & & & & & & \\
\hline k98.1.181.0 & 181.0 & oo packst & 2.28 & -11 & & & & 0.13 & -22.3 & \\
\hline k98.1.182.5 & 182.5 & oo grainst & 2.27 & -9.56 & 104.84 & & 1.86 & & & \\
\hline k98.1.183.0 & 183.0 & oo packst & 4.39 & -11.45 & 464.84 & & 0.2 & & & \\
\hline k98.1.184.5 & 184.5 & silt dol & 0.78 & -5.4 & 462.5 & & 1.97 & 0.13 & -19.8 & \\
\hline k98.1.189.3 & 189.3 & silt dol & & & & & & 0.14 & -21.5 & Krol B \\
\hline k98.1.206.0 & 206.0 & ch dol & -1.16 & -6.72 & & & & & & \\
\hline k98.1.219.0 & 219.0 & silt dol & -1.5 & -8.02 & 21.38 & & 30.38 & & & Surface 3 \\
\hline k98.1.220.0 & & silt dol & -7.89 & -7.79 & 143.26 & & 0.92 & 0.52 & -24.3 & \\
\hline k98.1.221.0 & & silt dol & 1.13 & -5.82 & & & & 0.31 & -23.1 & \\
\hline k98.1.222.0 & 222.0 & mud lm & & & & & & 0.27 & -25.0 & \\
\hline k98.1.223.0 & 223.0 & lm mudst & & & & & & 0.17 & -22.0 & \\
\hline
\end{tabular}


Table 1 (Continued)

\begin{tabular}{|c|c|c|c|c|c|c|c|c|c|c|}
\hline $\begin{array}{l}\text { Sample } \\
\text { number }\end{array}$ & $\begin{array}{l}\text { Position } \\
\text { (m) }\end{array}$ & Lithology* & $\begin{array}{l}\delta^{13} \mathrm{C} \\
(\% \circ, \mathrm{PDB})\end{array}$ & $\begin{array}{l}\delta^{18} \mathrm{O} \\
(\% o, \mathrm{PDB})\end{array}$ & $\begin{array}{l}\mathrm{Sr} \\
(\mathrm{ppm})\end{array}$ & $\begin{array}{l}\mathrm{Mn} \\
(\mathrm{ppm})\end{array}$ & $\begin{array}{l}\mathrm{Mn} / \\
\mathrm{Sr}\end{array}$ & $\begin{array}{l}\text { TOC } \\
(\mathrm{mg} / \mathrm{g})\end{array}$ & $\begin{array}{l}\delta^{13} \mathrm{C}_{\mathrm{TOC}} \\
(\% \circ, \mathrm{PDB})\end{array}$ & $\begin{array}{l}\text { Stratigraphic } \\
\text { units }\end{array}$ \\
\hline k98.1.224.6 & 224.6 & mud $1 \mathrm{~m}$ & -7.66 & -6.85 & & & & 0.46 & -24.3 & Krol C \\
\hline k98.1.226.0 & 226.0 & lm mudst & -7.8 & -5.67 & & & & 0.22 & -25.0 & \\
\hline k98.1.230.0 & 230.0 & intra packst & 1.15 & -9.4 & 64.56 & & 1.57 & 0.28 & -27.5 & \\
\hline k98.1.235.0 & 235.0 & mic dol & 1.32 & -10 & 36.98 & & & 0.41 & -22.6 & Surface 4 \\
\hline k98.1.245.0 & 245.0 & karstic br & -0.66 & -12.37 & 75.5 & & & 0.22 & -26.0 & \\
\hline k98.1.253.0 & 253.0 & karstic br & -0.18 & -12.65 & 49.1 & & 12.76 & & & \\
\hline k98.1.263.0 & 263.0 & karstic br & 2.66 & -8.64 & & & & 0.18 & -27.4 & \\
\hline k98.1.273.0 & 273.0 & karstic br & 1.89 & -8.3 & & & & 0.13 & -25.2 & \\
\hline k98.1.283.0 & 283.0 & karstic br & -0.79 & -12.68 & 69.35 & & 2.67 & 0.11 & -22.1 & \\
\hline k98.1.285.0 & 285.0 & oo grainst & 0.21 & -7.84 & & & & 0.15 & -21.7 & \\
\hline k98.1.295.0 & 295.0 & oo grainst & 0.2 & -4.56 & & & & 0.51 & -25.5 & \\
\hline k98.1.304.0 & 304.0 & lm mudst & -0.1 & -8.29 & & & & 0.16 & -23.7 & \\
\hline k98.1.318.0 & 318.0 & mic dol & 1.95 & -8.77 & 77.03 & & 2.61 & & & \\
\hline k98.1.326.0 & 326.0 & mic dol & 1.61 & -8.52 & & & & & & \\
\hline k98.1.336.0 & 336.0 & mic dol & & & 65.92 & & 4.75 & & & \\
\hline k98.1.352.0 & 352.0 & mic dol & 3.6 & -12.6 & & & & & & \\
\hline k98.1.363.0 & 363.0 & mic dol & 2.2 & -9.2 & & & & & & \\
\hline k98.1.381.0 & 381.0 & mic dol & 0.82 & -11.05 & 91.44 & & & & & \\
\hline k98.1.398.0 & 398.0 & mic dol & 2.09 & -9.11 & & & & & & Krol C \\
\hline k98.1.410.0 & 410.0 & intra grainst & 0.76 & -12.17 & & & & & & \\
\hline k98.1.430.0 & 430.0 & mic dol & 2.59 & -8.64 & & & & & & \\
\hline k98.1.448.0 & 448.0 & $\mathrm{mic} / \mathrm{ch}$ dol & 1.2 & -11.05 & & & & & & \\
\hline k98.1.465.0 & 465.0 & $\mathrm{mic} / \mathrm{ch}$ dol & 1.53 & -8.26 & & & & & & \\
\hline k98.1.490.0 & 490.0 & oo grainst & 1.34 & -7.39 & 64.43 & & 4.4 & & & \\
\hline k98.1.500.0 & 500.0 & oo grainst & 1.5 & -5.53 & 50.47 & & 7.19 & & & \\
\hline k98.1.508.0 & 508.0 & oo grainst & 1.3 & -5.14 & & & & & & \\
\hline k98.1.517.0 & 517.0 & ch dol & 1.49 & -5.82 & 49.63 & & & & & \\
\hline k98.1.520.0 & 520.0 & ch dol & 1.7 & -6.68 & & & & & & \\
\hline k98.1.530.0 & 530.0 & ch dol & 0.59 & -2.91 & 75.89 & & 5.88 & & & \\
\hline k98.1.545.0 & 545.0 & ch dol & -2.97 & -1.44 & 100.93 & & 2.8 & & & Surface 5 \\
\hline k98.1.553.0 & 553.0 & $1 \mathrm{~m} \mathrm{mud/cal}$ & -3.48 & -1.43 & & & & & & \\
\hline k98.1.556.0 & 556.0 & dol packst & -0.76 & -2.91 & & & & & & \\
\hline k98.1.559.0 & 559.0 & ch dol & -0.74 & -6.04 & 939.98 & & & & & \\
\hline k98.1.561.0 & 561.0 & ch dol & 0.03 & -5.13 & 78.33 & & 2.3 & & & \\
\hline k98.1.568.0 & 568.0 & lm mudst & 2.98 & -5 & 101.41 & & 8.86 & & & \\
\hline k98.1.586.0 & 586.0 & mic dol & 1.45 & -7.96 & 48.71 & & & & & \\
\hline k98.1.600.0 & 600.0 & intra packst & 1.74 & -7.94 & 69.75 & & 5.51 & & & \\
\hline k98.1.615.0 & 615.0 & intra packst & 1.28 & -5.63 & 42.02 & & & & & \\
\hline k98.1.630.0 & 630.0 & intra grainst & 2.12 & -5.37 & 104.98 & & & & & \\
\hline k98.1.645.0 & 645.0 & pel packst & 6.37 & -3.35 & 98.35 & & 3.8 & & & Krol D \\
\hline k98.1.660.0 & 660.0 & mic dol & 5.02 & -3.46 & 118.53 & & 3.22 & & & \\
\hline k98.1.693.0 & 693.0 & ch dol & 8.4 & -4.25 & 106.91 & & 0.77 & & & \\
\hline k98.1.706.0 & 706.0 & mic dol & 6.58 & -6.1 & 75.76 & & & & & \\
\hline k98.1.720.0 & 720.0 & ch dol & 3.43 & -4.34 & 87.07 & & 5.19 & & & \\
\hline k98.1.736.0 & 736.0 & ch dol & 4.22 & -4.88 & & & & & & \\
\hline k98.1.752.0 & 752.0 & ch dol & 4.02 & -4.11 & & & & & & \\
\hline k98.1.770.0 & 770.0 & ch dol & 3.63 & -3.26 & & & & & & \\
\hline k98.1.784.0 & 784.0 & ch dol & 4.04 & -4.38 & & & & & & \\
\hline k98.1.795.0 & 795.0 & ch dol & 4.12 & -3.44 & & & & & & \\
\hline k98.1.809.0 & 809.0 & ch dol & 5.06 & -5.03 & & & & & & \\
\hline \multicolumn{11}{|l|}{ M10 } \\
\hline M10-1 & 73.0 & mic dol & 1.26 & -8.91 & & & & & & Krol C \\
\hline M10-2 & 87.0 & mic dol & 2.24 & -9.81 & 119.83 & 131.4 & 1.1 & & & \\
\hline M10-3 & 98.0 & ch dol & 1.38 & -15.15 & 194.96 & 69.18 & 0.36 & & & \\
\hline M10-4 & 108.0 & ch dol & 1 & -13.88 & 191.22 & 101.58 & 0.53 & & & Surface 4 \\
\hline M10-5 & 116.0 & karstic br & 1.26 & -12.21 & & & & & & \\
\hline M10-7 & 129.0 & oo grainst & 1.21 & -12.31 & & & & & & Krol C \\
\hline
\end{tabular}


Table 1 (Continued)

\begin{tabular}{|c|c|c|c|c|c|c|c|c|c|c|}
\hline $\begin{array}{l}\text { Sample } \\
\text { number }\end{array}$ & $\begin{array}{l}\text { Position } \\
\text { (m) }\end{array}$ & Lithology ${ }^{*}$ & $\begin{array}{l}\delta^{13} \mathrm{C} \\
(\% o, \mathrm{PDB})\end{array}$ & $\begin{array}{l}\delta^{18} \mathrm{O} \\
(\% o, \mathrm{PDB})\end{array}$ & $\begin{array}{l}\mathrm{Sr} \\
(\mathrm{ppm})\end{array}$ & $\begin{array}{l}\mathrm{Mn} \\
(\mathrm{ppm})\end{array}$ & $\begin{array}{l}\mathrm{Mn} / \\
\mathrm{Sr}\end{array}$ & $\begin{array}{l}\text { TOC } \\
(\mathrm{mg} / \mathrm{g})\end{array}$ & $\begin{array}{l}\delta^{13} \mathrm{C}_{\mathrm{TOC}} \\
(\% \circ, \mathrm{PDB})\end{array}$ & $\begin{array}{l}\text { Stratigraphic } \\
\text { units }\end{array}$ \\
\hline M10-9 & 145.0 & mic dol & 0.95 & -6.36 & & & & & & \\
\hline M10-10 & 153.0 & lm mudst & -3.78 & -6.16 & & & & & & \\
\hline M10-11 & 162.0 & Wackestone & -2.77 & -5.64 & & & & & & \\
\hline \multicolumn{11}{|c|}{ 4. Samples from Nigalidhar syncline } \\
\hline \multicolumn{11}{|l|}{ N1-N3 } \\
\hline N3-1 & 13.0 & lm mudst & 4.02 & -12.34 & & & & & & Krol A \\
\hline N3-2 & 25.0 & $1 \mathrm{~m}$ mudst & 4.15 & -12.07 & 845.62 & 443.59 & 0.53 & & & \\
\hline N3-3 & 41.0 & mud $\operatorname{lm}$ & 2.86 & -11.83 & 318.14 & 351.5 & 1.11 & & & \\
\hline N3-4 & 48.0 & silt dol & 3.76 & -11.95 & & & & & & Krol B \\
\hline $\mathrm{N} 1-1$ & 52.0 & silt dol & 1.03 & -7.35 & & & & 0.1 & -16.7 & \\
\hline $\mathrm{N} 1-2$ & 57.0 & silt/ch dol & 2.02 & -6.51 & & & & 0.08 & -17.6 & \\
\hline $\mathrm{N} 1-3$ & 61.0 & silt/ch dol & 2.76 & -5.16 & & & & 0.14 & -20.8 & \\
\hline N1-4 & 68.0 & silt/ch dol & & & & & & 0.15 & -22.8 & \\
\hline N1-5 & 72.0 & silt/ch dol & 3.1 & -5.85 & & & & 0.09 & -18.2 & \\
\hline N1-6 & 77.0 & silt/ch dol & -1.99 & -6.61 & & & & 0.11 & -20.4 & Surface 3 \\
\hline N1-7 & 79.0 & dol wackest & 0.98 & -7.73 & 118.35 & 898.23 & 7.59 & 0.13 & -22.1 & \\
\hline N1-8 & 84.0 & dol packst & 0.45 & -6.7 & & & & 0.24 & -24.6 & \\
\hline N1-9 & 86.0 & mud dol & -0.98 & -6.75 & & & & 0.07 & & \\
\hline N1-10 & 89.0 & lm mudst & -1.67 & -6.71 & & & & 0.08 & -20.1 & Krol C \\
\hline N1-12 & 110.5 & intra grainst & 1.44 & -9.79 & & & & & & \\
\hline N1-13 & 122.0 & intra packst & 0.77 & -15.44 & & & & & & \\
\hline N1-14 & 138.0 & $\mathrm{mic} / \mathrm{ch}$ dol & 0.06 & -12.14 & 47.12 & 83.12 & 1.76 & & & \\
\hline N1-15 & 141.5 & ch dol & 1.44 & -8.18 & 113.48 & 180 & 1.59 & & -30.3 & \\
\hline N1-16 & 148.0 & ch dol & 1.44 & -10.72 & & & & 0.14 & -28.3 & Surface 4 \\
\hline N1-17 & 153.0 & karstic br & -0.08 & -13.72 & 23.6 & 866.47 & 36.71 & & & \\
\hline N1-18 & 158.5 & oo grainst & 1.76 & -5 & & & & & & \\
\hline N1-19 & 165.5 & oo grainst & 1.82 & -4.19 & & & & 0.1 & -26.1 & \\
\hline N1-20 & 174.0 & oo grainst & 2.09 & -3.79 & & & & & & \\
\hline N1-21 & 182.0 & oo grainst & 2.48 & -4.32 & & & & & & \\
\hline $\mathrm{N} 1-22$ & 183.0 & oo grainst & -0.44 & -5.27 & & & & & & \\
\hline N1-23 & 187.0 & $1 \mathrm{~m}$ mudst & -9.35 & -3.85 & & & & 0.3 & -28.5 & \\
\hline N1-24 & 190.0 & Im mudst & -0.04 & -2.52 & 52.96 & 47.4 & 0.9 & & & Krol C \\
\hline N1-25 & 196.0 & lm mudst & -1.2 & -1.85 & 68.78 & 190.93 & 2.78 & & & \\
\hline N1-26 & 198.0 & dol wackest & -2.46 & -2.63 & & & & 0.38 & -24.5 & \\
\hline N1-27 & 200.0 & dol wackest & 0.51 & -1.67 & & & & 0.55 & & \\
\hline N1-28 & 201.5 & dol wackest & -1.84 & -3.73 & 97.51 & 166.61 & 1.71 & 0.81 & -39.3 & \\
\hline N1-29 & 204.0 & dol wackest & -0.92 & -4.26 & 76.15 & 235.97 & 3.1 & & & \\
\hline $\mathrm{N} 1-30$ & 206.0 & lm mudst & -1.9 & -0.45 & & & & 0.35 & -28.9 & \\
\hline N1-31 & 211.0 & $1 \mathrm{~m}$ mudst & -2.42 & 0.23 & & & & & & \\
\hline $\mathrm{N} 1-32$ & 214.0 & lm mudst & -1.11 & -3.24 & 87.87 & 287.59 & 3.27 & 0.26 & -37.9 & \\
\hline N1-33 & 219.0 & $1 \mathrm{~m}$ mudst & -2.71 & -6.44 & & & & 0.2 & -27.4 & Krol C \\
\hline $\mathrm{N} 1-34$ & 224.0 & dol packst & 1.9 & -2.07 & 123.26 & 51.11 & 0.42 & 0.41 & -27.8 & \\
\hline $\mathrm{N} 1-35$ & 233.0 & dol wackest & 1.3 & -3.61 & & & & & & \\
\hline N1-36 & 240.2 & oo grainst & 3.8 & -4.02 & 97.94 & 25.93 & 0.27 & 0.21 & -21.4 & \\
\hline N1-37 & 248.0 & mic dol & 3.45 & -3.68 & 124.1 & 6.21 & 0.05 & 0.43 & -26.5 & \\
\hline $\mathrm{N} 1-38$ & 258.0 & mic dol & 4.06 & -5.46 & & & & 0.9 & -24.5 & \\
\hline N1-39 & 270.0 & $\mathrm{mic} / \mathrm{ch}$ dol & 2.69 & -4.06 & & & & & & \\
\hline N1-40 & 281.0 & dol wackest & 9.18 & -3.45 & & & & 0.18 & -22.1 & \\
\hline N1-41 & 288.0 & dol wackest & 2.27 & -2.57 & & & & 0.27 & -20.9 & \\
\hline $\mathrm{N} 1-42$ & 302.0 & intra packst & 2.45 & -3.33 & & & & 0.57 & -23.4 & \\
\hline \multicolumn{11}{|c|}{ Main section } \\
\hline N1 & 4.5 & mud $\operatorname{lm}$ & 3.64 & -5.1 & & & & & & Krol A \\
\hline $\mathrm{N} 2$ & 11.0 & silt dol & 3.07 & -7.28 & & & & & & \\
\hline $\mathrm{N} 3$ & 14.0 & silt dol & 0.83 & -5.72 & & & & & & \\
\hline N4 & 18.5 & $\mathrm{ch} / \mathrm{silt}$ dol & 2.59 & -7.35 & & & & & & \\
\hline N5 & 21.5 & $\mathrm{ch} / \mathrm{silt}$ dol & 1.63 & -7.93 & & & & & & Krol B \\
\hline N6 & 23.0 & $\mathrm{ch} / \mathrm{silt}$ dol & 0.47 & -6.03 & & & & & & \\
\hline
\end{tabular}


Table 1 (Continued)

\begin{tabular}{|c|c|c|c|c|c|c|c|c|c|c|}
\hline $\begin{array}{l}\text { Sample } \\
\text { number }\end{array}$ & $\begin{array}{l}\text { Position } \\
\text { (m) }\end{array}$ & Lithology ${ }^{*}$ & $\begin{array}{l}\delta^{13} \mathrm{C} \\
(\% o, \mathrm{PDB})\end{array}$ & $\begin{array}{l}\delta^{18} \mathrm{O} \\
(\% o, \mathrm{PDB})\end{array}$ & $\begin{array}{l}\mathrm{Sr} \\
(\mathrm{ppm})\end{array}$ & $\begin{array}{l}\mathrm{Mn} \\
(\mathrm{ppm})\end{array}$ & $\begin{array}{l}\mathrm{Mn} / \\
\mathrm{Sr}\end{array}$ & $\begin{array}{l}\text { TOC } \\
(\mathrm{mg} / \mathrm{g})\end{array}$ & $\begin{array}{l}\delta^{13} \mathrm{C}_{\mathrm{TOC}} \\
(\%,, \mathrm{PDB})\end{array}$ & $\begin{array}{l}\text { Stratigraphic } \\
\text { units }\end{array}$ \\
\hline N7 & 26.5 & $\mathrm{ch} / \mathrm{silt}$ dol & -0.2 & -5.88 & & & & & & \\
\hline N9 & 32.0 & silt dol & 0.96 & -6.19 & & & & & & \\
\hline N10 & 37.0 & silt dol & -1.31 & -5.94 & & & & & & Surface 3 \\
\hline N11 & 38.3 & mud $1 \mathrm{~m}$ & -0.87 & -7.17 & & & & & & \\
\hline $\mathrm{N} 12$ & 39.5 & dol wackest & -2.03 & -6.36 & & & & & & \\
\hline N13 & 42.0 & dol packst & -8.8 & -6.63 & & & & & & \\
\hline N14 & 44.0 & dol packst & -7.89 & -4.95 & & & & & & \\
\hline N15 & 47.5 & mud dol & -8.1 & -4.47 & & & & & & \\
\hline N16 & 50.0 & $1 \mathrm{~m}$ mudst & -7.98 & -6.96 & & & & & & \\
\hline N17 & 59.0 & lm mudst & 1.23 & -10.27 & & & & & & \\
\hline N18 & 63.0 & $1 \mathrm{~m}$ mudst & 1.7 & -9.52 & & & & & & Krol C \\
\hline N19 & 68.0 & lm mudst & 1.62 & -6.89 & & & & & & \\
\hline $\mathrm{N} 20$ & 69.0 & intra grainst & 1.87 & -8.78 & & & & & & \\
\hline N21 & 75.5 & dol wackest & 1.22 & -13.51 & & & & & & \\
\hline $\mathrm{N} 22$ & 82.5 & intra grainst & 1.47 & -18.18 & & & & & & \\
\hline $\mathrm{N} 23$ & 85.0 & dol wackest & 1.42 & -15.19 & & & & & & \\
\hline $\mathrm{N} 24$ & 89.0 & mass/vug dol & 0.69 & -14.82 & & & & & & \\
\hline $\mathrm{N} 25$ & 92.5 & mass/vug dol & 1.17 & -4.92 & & & & & & \\
\hline N26 & 97.0 & mass/vug dol & 1.48 & -8.88 & & & & & & \\
\hline $\mathrm{N} 27$ & 99.0 & mass/vug dol & 1.5 & -8.27 & & & & & & \\
\hline $\mathrm{N} 29$ & 104.0 & karstic br & -0.52 & -15.1 & & & & & & Surface 4 \\
\hline N30 & 106.0 & karstic br & 1.14 & -15.39 & & & & & & \\
\hline N31 & 108.0 & karstic br & 0.4 & -5.07 & & & & & & \\
\hline N32 & 114.0 & karstic br & 1.72 & -11.16 & & & & & & \\
\hline N34 & 119.0 & oo grainst & 2.65 & -2.77 & & & & & & Krol C \\
\hline N35 & 122.0 & oo grainst & 0.05 & -2.58 & & & & & & \\
\hline N36 & 128.5 & oo grainst & 1 & -1.49 & & & & & & \\
\hline N37 & 139.0 & oo grainst & 1.09 & -1.48 & & & & & & \\
\hline $8 N-3$ & 177.0 & $1 \mathrm{~m}$ mudst & 7.09 & -2.72 & & & & 0.18 & -27.2 & \\
\hline $8 \mathrm{~N}-4$ & 187.0 & dol packst & & & & & & & & \\
\hline $8 N-5$ & 198.0 & mic dol & 3.89 & -4.58 & & & & 0.43 & -27.0 & \\
\hline $8 N-6$ & 206.5 & dol packst & 8.24 & -2.49 & 141.21 & 36.04 & 0.26 & 0.23 & -22.0 & \\
\hline $8 \mathrm{~N}-7$ & 219.0 & mic dol & 1.52 & -1.94 & & & & 0.93 & & \\
\hline $8 \mathrm{~N}-8$ & 233.0 & mic dol & & & & & & & -24.2 & \\
\hline $8 N-9$ & 241.0 & dol wackest & & & 116.23 & 113.72 & 0.98 & & & \\
\hline $8 \mathrm{~N}-10$ & 247.0 & dol wackest & 2.11 & -2.72 & & & & 0.05 & -27.7 & \\
\hline $8 \mathrm{~N}-11$ & 254.0 & dol wackest & 1.74 & -0.75 & & & & & & \\
\hline $8 \mathrm{~N}-12$ & 277.0 & dol wackest & 2.31 & -2.23 & 158.02 & 141.14 & 0.89 & 0.27 & -23.7 & \\
\hline $8 \mathrm{~N}-14$ & 301.5 & $\mathrm{ch} /$ silt dol & -1.15 & -2.17 & 94.43 & 149.99 & 1.59 & & & \\
\hline $8 \mathrm{~N}-15$ & 318.0 & ch dol & 1.71 & -1.96 & & & & & & Surface 5 \\
\hline $8 \mathrm{~N}-16$ & 328.5 & lm mudst & -3.88 & -7.61 & & & & & & \\
\hline $8 \mathrm{~N}-17$ & 333.0 & dol packst & -0.64 & -4.24 & & & & 0.22 & -27.2 & \\
\hline $8 \mathrm{~N}-19$ & 357.0 & lm mudst & -4.45 & -3.54 & & & & 0.4 & -25.4 & \\
\hline $8 \mathrm{~N}-20$ & 360.5 & oo grainst & -3.58 & -6.07 & & & & & & \\
\hline $8 \mathrm{~N}-21$ & 364.0 & oo grainst & -2.95 & -6.28 & 23.35 & 192.34 & 8.24 & & & \\
\hline $8 \mathrm{~N}-22$ & 378.0 & mic dol & -2.03 & -2.46 & 76.35 & 213.15 & 2.8 & 0.82 & -27.2 & Krol D \\
\hline $8 \mathrm{~N}-23$ & 392.5 & mic dol & & & & & & 0.16 & -23.3 & \\
\hline $8 \mathrm{~N}-24$ & 411.0 & dol wackest & 3.22 & -4.06 & & & & & & \\
\hline $8 \mathrm{~N}-25$ & 414.0 & $\mathrm{mic} / \mathrm{ch}$ dol & 3.4 & -0.58 & & & & & & \\
\hline $8 \mathrm{~N}-26$ & 427.0 & dol wackest & 3.5 & -3.18 & & & & & & \\
\hline $8 \mathrm{~N}-27$ & 442.5 & dol wackest & 3.41 & -0.62 & 33.88 & 61.39 & 1.81 & & & \\
\hline $8 \mathrm{~N}-28$ & 457.0 & oo grainst & 3.9 & -0.55 & 17.1 & 53.9 & 3.15 & & & \\
\hline $8 \mathrm{~N}-29$ & 464.0 & mic dol & 2.59 & -1.78 & 43.36 & 44.62 & 1.03 & & & Krol D \\
\hline $8 \mathrm{~N}-30$ & 482.5 & mic dol & 2.47 & -1.23 & & & & & & \\
\hline $8 \mathrm{~N}-31$ & 497.0 & mic dol & 2.58 & -3.85 & 67.47 & 88.44 & 1.31 & & & \\
\hline $8 \mathrm{~N}-32$ & 509.0 & mic dol & 1.78 & -5.3 & & & & & & \\
\hline $8 N-33$ & 524.0 & mic dol & 3.02 & -3.09 & 45.08 & 79.53 & 1.76 & & & \\
\hline $8 N-34$ & 540.0 & mic dol & 1.26 & -4.26 & & & & & & \\
\hline
\end{tabular}


Table 1 (Continued)

\begin{tabular}{|c|c|c|c|c|c|c|c|c|c|c|}
\hline $\begin{array}{l}\text { Sample } \\
\text { number }\end{array}$ & $\begin{array}{l}\text { Position } \\
(\mathrm{m})\end{array}$ & Lithology ${ }^{*}$ & $\begin{array}{l}\delta^{13} \mathrm{C} \\
(\% o, \mathrm{PDB})\end{array}$ & $\begin{array}{l}\delta^{18} \mathrm{O} \\
(\% \circ, \mathrm{PDB})\end{array}$ & $\begin{array}{l}\mathrm{Sr} \\
(\mathrm{ppm})\end{array}$ & $\begin{array}{l}\mathrm{Mn} \\
(\mathrm{ppm})\end{array}$ & $\begin{array}{l}\mathrm{Mn} / \\
\mathrm{Sr}\end{array}$ & $\begin{array}{l}\text { TOC } \\
(\mathrm{mg} / \mathrm{g})\end{array}$ & $\begin{array}{l}\delta^{13} \mathrm{C}_{\mathrm{TOC}} \\
(\%, \mathrm{PDB})\end{array}$ & $\begin{array}{l}\text { Stratigraphic } \\
\text { units }\end{array}$ \\
\hline $8 \mathrm{~N}-35$ & 555.0 & mic dol & 1.62 & -3.63 & 74.04 & 74.1 & 1 & & & \\
\hline $8 N-36$ & 573.0 & mic dol & 1.19 & -1.86 & 42.7 & 54.41 & 1.27 & & & \\
\hline $8 \mathrm{~N}-37$ & 590.0 & mic dol & 1.45 & -1.53 & 38.97 & 48.14 & 1.24 & & & \\
\hline $8 \mathrm{~N}-38$ & 608.0 & mic dol & 0.81 & -0.92 & & & & & & \\
\hline $8 N-39$ & 630.0 & mic dol & 2 & -3.22 & & & & & & \\
\hline $8 \mathrm{~N}-40$ & 651.0 & dol packst & 1.22 & -1.68 & 49.27 & 50.71 & 1.03 & & & \\
\hline $8 \mathrm{~N}-41$ & 674.0 & mic dol & 1.75 & -2.03 & & & & & & \\
\hline $8 \mathrm{~N}-42$ & 717.0 & mic dol & 2.27 & -1.31 & 44.57 & 78.63 & 1.76 & & & \\
\hline $8 \mathrm{~N}-43$ & 743.0 & mic dol & 1.07 & -2.78 & 44.57 & 89.56 & 2.01 & & & \\
\hline $8 \mathrm{~N}-44$ & 768.0 & $\mathrm{mic} / \mathrm{ch}$ dol & 0.75 & -1.92 & 21.56 & 67.96 & 3.15 & & & Surface 6 \\
\hline \multicolumn{11}{|l|}{ N5 } \\
\hline N5-1 & 273.0 & karstic br & 1.92 & -10.98 & & & & & & \\
\hline N5-3 & 281.0 & oo grainst & 0.65 & -7.09 & & & & 0.12 & -25.1 & \\
\hline N5-4 & 288.0 & oo grainst & 2.47 & -8.29 & 66.6 & 12.9 & 0.19 & 0.25 & -28.8 & \\
\hline N5-5 & 297.0 & oo grainst & 2.27 & -3.95 & & & & & & \\
\hline N5-6 & 306.0 & oo grainst & 2.28 & -4.79 & 71.65 & & & & & Krol C \\
\hline N5-7 & 310.0 & dol packst & 2.49 & -4.02 & 91.52 & 24.23 & 0.27 & & & \\
\hline N5-8 & 313.0 & mic dol & 2.1 & -3.64 & & & & & & \\
\hline N5-9 & 318.5 & mic dol & & & & & & 0.18 & -21.8 & \\
\hline N5-13 & 331.0 & oo grainst & -2.4 & -8.03 & & & & & & \\
\hline N5-14 & 336.0 & strom floatst & 1.76 & -3.78 & 88.98 & 23.56 & 0.27 & & & \\
\hline N5-15 & 339.0 & mic dol & -0.01 & -6.3 & 170.55 & 48.88 & 0.29 & 0.51 & -25.6 & \\
\hline N5-18 & 347.5 & strom floatst & -8.3 & -2.51 & & & & & & \\
\hline N5-19 & 357.0 & mic dol & -3.8 & -1.5 & & & & 1.24 & -27.1 & \\
\hline \multicolumn{11}{|l|}{ NK } \\
\hline NK-1 & 72.0 & mud dol & -3.28 & -8.24 & & & & & & \\
\hline NK-2 & 72.8 & mud dol & -3.46 & -8.79 & & & & & & \\
\hline NK-3 & 73.5 & mud dol & -2.56 & -8.98 & & & & & & \\
\hline NK-4 & 74.2 & mud dol & -3.58 & -8.87 & & & & & & $\begin{array}{l}\text { "Cap } \\
\text { carbonate" }\end{array}$ \\
\hline NK-5 & 75.1 & mud dol & -3.7 & -9.48 & 70 & 894.82 & 12.78 & & & \\
\hline NK-6 & 76.0 & mud dol & -2.62 & -9.08 & & & & & & \\
\hline NK-7 & 76.8 & mud dol & -3.83 & -8.93 & & & & & & \\
\hline \multicolumn{11}{|c|}{$\begin{array}{l}\text { 5. Samples from Solan } \\
\text { s4 }\end{array}$} \\
\hline s4-3 & 118.5 & mud dol & -2.08 & -3.24 & & & & & & \\
\hline s4-4 & 153.0 & $\operatorname{lm}$ mudst & 5.32 & -3.42 & & & & & & \\
\hline s4-5 & 157.0 & mud $1 \mathrm{~m}$ & 3.09 & -10.11 & & & & & & \\
\hline$s 4-6$ & 161.0 & lm mudst & 3.77 & -10.88 & 180.57 & 446.97 & 2.48 & & & Krol A \\
\hline s4-7 & 167.0 & mud $1 \mathrm{~m}$ & 4.22 & -10.45 & 102.71 & 677.24 & 6.6 & & & \\
\hline s4-8 & 178.0 & lm mudst & 4.22 & -10.95 & 246.7 & 304.61 & 1.24 & & & \\
\hline s4-9 & 189.0 & lm mudst & 3.46 & -11.92 & & & & & & \\
\hline \multicolumn{11}{|l|}{ k97.2-3 } \\
\hline k97.2.72.5 & 72.5 & org sh & & & & & & & & \\
\hline k97.2.135.3 & 135.3 & mud $1 \mathrm{~m}$ & 1.76 & -3.24 & & & & & & \\
\hline k97.2.135.5 & 135.5 & mud $1 \mathrm{~m}$ & 2.39 & -2.76 & 40.29 & 443 & 11 & & & Krol A \\
\hline k97.2.137.0 & 137.0 & mud lm & 3.15 & -5.64 & 30.14 & 25.87 & 0.86 & & & \\
\hline k97.2.193.9 & 193.9 & lm mudst & 3.38 & -10.1 & 225.4 & 403.43 & 1.79 & & & \\
\hline k97.2.201.5 & 201.5 & mud lm & 3.12 & -9 & & & & & & \\
\hline k97.2.346.1 & 346.1 & silt dol & -0.36 & -6.39 & & & & & & $\begin{array}{l}\text { Krol B/Krol } \\
\text { C? }\end{array}$ \\
\hline k97.2.346.5 & 346.5 & silt dol & -1.71 & -6.82 & & & & 0.13 & -23.0 & \\
\hline k97.2.347.1 & 347.1 & silt dol & -1.73 & -6.45 & 69.93 & 617.3 & 8.83 & 0.15 & -25.4 & \\
\hline k97.2.347.6 & 347.6 & silt dol & -4.2 & -6.46 & 102.09 & 381.13 & 3.73 & 0.13 & -23.6 & \\
\hline k97.2.348.1 & 348.1 & silt dol & -5.74 & -7.05 & 78.61 & 274.35 & 3.49 & 0.09 & -21.1 & \\
\hline k97.2.348.6 & 348.6 & silt dol & -6.85 & -7.13 & 75.41 & 245.06 & 3.25 & & & \\
\hline
\end{tabular}


Table 1 (Continued)

\begin{tabular}{|c|c|c|c|c|c|c|c|c|c|c|}
\hline $\begin{array}{l}\text { Sample } \\
\text { number }\end{array}$ & $\begin{array}{l}\text { Position } \\
\text { (m) }\end{array}$ & Lithology* & $\begin{array}{l}\delta^{13} \mathrm{C} \\
(\% o, \mathrm{PDB})\end{array}$ & $\begin{array}{l}\delta^{18} \mathrm{O} \\
(\% \circ, \mathrm{PDB})\end{array}$ & $\begin{array}{l}\mathrm{Sr} \\
(\mathrm{ppm})\end{array}$ & $\begin{array}{l}\mathrm{Mn} \\
(\mathrm{ppm})\end{array}$ & $\begin{array}{l}\mathrm{Mn} / \\
\mathrm{Sr}\end{array}$ & $\begin{array}{l}\text { TOC } \\
(\mathrm{mg} / \mathrm{g})\end{array}$ & $\begin{array}{l}\delta^{13} \mathrm{C}_{\mathrm{TOC}} \\
(\% \circ, \mathrm{PDB})\end{array}$ & $\begin{array}{l}\text { Stratigraphic } \\
\text { units }\end{array}$ \\
\hline k97.2.349.1 & 349.1 & silt dol & -8.69 & -7.25 & 64.93 & 453.24 & 6.98 & 0.08 & -21.9 & \\
\hline k97.2.350.1 & 350.1 & silt dol & -9.72 & -6.63 & 46.3 & 517.4 & 11.17 & 0.23 & -24.6 & Surface $3 ?$ \\
\hline k97.2.367.7 & 367.7 & mic dol & 2.21 & -7.39 & 40.73 & 106.22 & 2.61 & & & \\
\hline k97.2.372.7 & 372.7 & mic dol & 2.29 & -5.23 & 41.87 & 76.3 & 1.82 & & & \\
\hline k97.2.377.7 & 377.7 & mic dol & 2.03 & -7.92 & & & & & & Krol C \\
\hline k97.2.382.7 & 382.7 & mic dol & 2.02 & -8.77 & 43.86 & 70.34 & 1.6 & & & \\
\hline k97.3.3.0 & 403.0 & $\mathrm{mic} / \mathrm{ch}$ dol & 1.76 & -5.95 & & & & & & \\
\hline k98.3.8.0 & 408.0 & $\mathrm{mic} / \mathrm{ch}$ dol & 2.31 & -6.46 & 43.64 & 39.11 & 0.9 & & & \\
\hline k97.3.20.0 & 420.0 & $\mathrm{mic} / \mathrm{ch}$ dol & -0.62 & -6.58 & 95.69 & 15.09 & 0.16 & & & Surface 4 \\
\hline k97.3.30.0 & 430.0 & karstic br & 2.9 & -3.07 & 71.03 & 8.7 & 0.12 & & & Krol C \\
\hline k97.3.40.0 & 440.0 & oo packst & -1.04 & -9 & 69.87 & 35.75 & 0.51 & & & \\
\hline k97.3.50.0 & 450.0 & oo packst & 2.51 & -2.99 & 44.45 & 33.52 & 0.75 & & & \\
\hline k97.3.60.0 & 460.0 & mic dol & 1.66 & -5.26 & 45.5 & 34.31 & 0.75 & & & \\
\hline k97.3.70.0 & 470.0 & mic dol & 2.38 & -3.37 & & & & & & \\
\hline \multicolumn{11}{|l|}{ k97.4 } \\
\hline k97.4.47.5 & 47.5 & mud dol & -3.63 & -5.74 & 138.92 & 326.44 & 2.35 & & & Krol A \\
\hline k97.4.57.7 & 57.7 & mud dol & -9.6 & -4.35 & & & & & & \\
\hline k97.4.116.2 & 116.2 & mud lm & 4.21 & -11.25 & & & & & & \\
\hline k97.4.119.2 & 119.2 & mud $1 \mathrm{~m}$ & 3.04 & -10.14 & & & & & & \\
\hline k97.4.131.9 & 131.9 & mud lm & 3.75 & -9.57 & 20.37 & 369.71 & 18.15 & 1.43 & -28.0 & \\
\hline k97.4.220.1 & 220.1 & silt dol & 2.94 & -4.97 & 97.9 & 2683.08 & 27.41 & 0.22 & -23.5 & Krol B \\
\hline k97.4.226.1 & 226.1 & silt dol & 1.36 & -6.45 & 373.89 & 625.38 & 1.67 & 0.23 & -23.2 & \\
\hline k97.4.227.6 & 227.6 & silt dol & -0.03 & -6.29 & 157.41 & 1963.22 & 12.47 & 1.08 & & \\
\hline k97.4.231.4 & 231.4 & silt dol & -2.06 & -2.56 & 75.94 & 283.95 & 3.74 & 0.45 & -24.9 & \\
\hline k97.4.238.6 & 238.6 & silt dol & 1.92 & -6.58 & 113.35 & 1236.02 & 10.9 & 0.36 & -23.6 & \\
\hline k97.4.239.6 & 239.6 & silt dol & 2.06 & -7.73 & 43.65 & 137.55 & 3.15 & 0.6 & -30.6 & Surface 3 \\
\hline k97.4.252.1 & 252.1 & mic dol & 1.88 & -7.1 & 46.76 & 615.12 & 13.16 & 0.41 & -24.6 & Krol C \\
\hline k97.4.267.1 & 267.1 & mic dol & 1.61 & -6.37 & 64.53 & 208.46 & 3.23 & 0.28 & -27.1 & \\
\hline k97.4.272.1 & 272.1 & mic dol & 1.44 & -10.46 & 102.18 & 248.68 & 2.44 & 0.28 & -24.9 & Surface 4 \\
\hline k97.4.277.1 & 277.1 & oo grainst & 2.11 & -6.6 & 53.67 & 74.49 & 1.39 & 0.4 & -24.0 & \\
\hline k97.4.282.1 & 282.1 & oo packst & 2.15 & -8.05 & 20.01 & 162.741 & 8.14 & 0.48 & -30.7 & \\
\hline k97.4.287.1 & 287.1 & oo grainst & 1.87 & -7.88 & & & & 0.49 & -22.4 & \\
\hline k97.4.292.1 & 292.1 & oo packst & 1.68 & -8.53 & & & & 0.52 & -30.9 & Krol C \\
\hline k97.4.297.1 & 297.1 & oo grainst & 2.15 & -5.07 & & & & 0.63 & -31.1 & \\
\hline k97.4.302.1 & 302.1 & oo grainst & 1.59 & -8.47 & 43.95 & 67.52 & 1.53 & 0.57 & -30.9 & \\
\hline \multicolumn{11}{|l|}{ s3 } \\
\hline s3-1 & 128.0 & mud $1 \mathrm{~m}$ & 1.36 & -8.71 & 441.87 & 437.4 & 0.99 & & & Krol A \\
\hline s3-2 & 153.0 & mud lm & 3.8 & -11.22 & & & & & & \\
\hline s3-3 & 169.0 & mud lm & 3.23 & -8.22 & & & & & & \\
\hline s3-4 & 186.0 & mud lm & 1.66 & -10.83 & & & & & & \\
\hline s3-5 & 198.0 & silt dol & 5.43 & -5.15 & 80.75 & 455.67 & 5.64 & & & \\
\hline s3-6 & 220.0 & silt dol & 4.24 & -6.75 & 128.38 & 841.87 & 6.56 & 0.16 & -19.5 & \\
\hline s3-7 & 229.0 & silt dol & 3.87 & -6.55 & 29.2 & 306.03 & 10.48 & & & Krol B \\
\hline s3-10 & 246.0 & silt dol & -0.86 & -6.73 & 158.29 & 383.98 & 2.43 & & & \\
\hline s3-11 & 248.0 & silt dol & -0.02 & -6.11 & & & & 0.29 & -26.3 & Surface 3 \\
\hline s3-12 & 252.0 & mud lm & -0.56 & -5.99 & 31.21 & 33.71 & 1.08 & & & \\
\hline s3-14 & 257.0 & dol packst & -0.14 & -6.29 & 80.52 & 440.81 & 5.48 & 0.29 & -25.3 & \\
\hline s3-15 & 277.0 & mud lm & 0.29 & -7.85 & 166.97 & 821.86 & 4.92 & 1.45 & -25.8 & Krol C \\
\hline s3-16 & 280.0 & dol packst & 1.16 & -9.79 & & & & & & \\
\hline s3-17 & 285.0 & mic dol & 0.68 & -10.3 & & & & 1.85 & -27.0 & Surface 4 \\
\hline s3-18 & 297.0 & oo grainst & 1.35 & -11.81 & & & & & & \\
\hline s3-19 & 318.0 & mic dol & 1.19 & -11.29 & & & & 0.62 & -29.4 & \\
\hline s3-20 & 339.0 & mic dol & 1.83 & -10.95 & 88.75 & 374.2 & 4.22 & & & Krol C \\
\hline s3-21 & 378.0 & oo grainst & 1.71 & -11.21 & & & & 0.94 & -29.1 & \\
\hline s3-22 & 398.0 & mic dol & 2.28 & -6.69 & & & & & & \\
\hline s3-23 & 416.0 & mic dol & 2.13 & -4.03 & 86.52 & 381.23 & 4.41 & 0.19 & -29.0 & \\
\hline s3-24 & 437.0 & intra grainst & 1.52 & -5.42 & & & & & & \\
\hline
\end{tabular}


Table 1 (Continued)

\begin{tabular}{|c|c|c|c|c|c|c|c|c|c|c|}
\hline $\begin{array}{l}\text { Sample } \\
\text { number }\end{array}$ & $\begin{array}{l}\text { Position } \\
\text { (m) }\end{array}$ & Lithology ${ }^{*}$ & $\begin{array}{l}\delta^{13} \mathrm{C} \\
(\% o, \mathrm{PDB})\end{array}$ & $\begin{array}{l}\delta^{18} \mathrm{O} \\
(\% \circ, \mathrm{PDB})\end{array}$ & $\begin{array}{l}\mathrm{Sr} \\
(\mathrm{ppm})\end{array}$ & $\begin{array}{l}\mathrm{Mn} \\
(\mathrm{ppm})\end{array}$ & $\begin{array}{l}\mathrm{Mn} / \\
\mathrm{Sr}\end{array}$ & $\begin{array}{l}\text { TOC } \\
(\mathrm{mg} / \mathrm{g})\end{array}$ & $\begin{array}{l}\delta^{13} \mathrm{C}_{\mathrm{TOC}} \\
(\% o, \mathrm{PDB})\end{array}$ & $\begin{array}{l}\text { Stratigraphic } \\
\text { units }\end{array}$ \\
\hline s3-25 & 447.0 & pel packst & 2.69 & -5.07 & 91.01 & 47.11 & 0.52 & 0.29 & -30.3 & \\
\hline s3-26 & 457.0 & pel packst & 2.05 & -3.22 & 226.68 & 116.83 & 0.52 & & & \\
\hline s3-27 & 468.0 & $\mathrm{mic} / \mathrm{ch}$ dol & -7.58 & -4.74 & & & & 0.57 & -26.9 & \\
\hline s3-28 & 490.0 & $\mathrm{mic} / \mathrm{ch}$ dol & -5.26 & -3.03 & & & & & & \\
\hline s3-29 & 504.0 & $\mathrm{mic} / \mathrm{ch} \mathrm{dol}$ & 0.77 & -2.26 & 108.79 & 451.62 & 4.15 & 0.2 & & Surface 5 \\
\hline s3-30 & 516.0 & silt dol & -0.23 & -3.87 & 168.33 & 371.29 & 2.21 & & & \\
\hline s3-31 & 554.0 & dol packst & -1.05 & -5.62 & & & & & & \\
\hline s3-32 & 582.0 & dol packst & -2.41 & -2.25 & & & & & & \\
\hline s3-33 & 608.0 & mic dol & -2.48 & -0.96 & & & & & & Krol D \\
\hline s3-34 & 635.0 & intra grainst & 2.91 & -1.12 & & & & & & \\
\hline s3-35 & 652.0 & mic dol & 6.56 & -5.3 & 113.6 & 46.23 & 0.41 & & & \\
\hline s3-36 & 664.0 & mic dol & 6.37 & -4.47 & 200.62 & 68.75 & 0.34 & & & \\
\hline s3-37 & 679.0 & mic dol & 5.29 & -7.23 & & & & & & \\
\hline
\end{tabular}

${ }^{*}$ Explanation for lithology abbreviations: $\mathrm{bk}=$ black; $\mathrm{br}=$ brecciated; cal = calcrete; $\mathrm{ch}=$ cherty or chert; det = detritus; dol = dolomite or

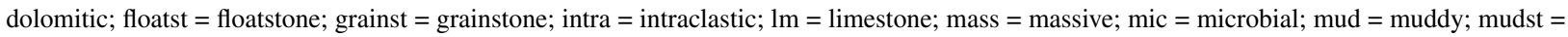
mudstone; oo = oolitic; org = organic-rich packst = packstone; pel = peloidal phosph = phosphorite silt $=$ silty $;$ sand $=$ sandy $;$ sh = shale $;$ strom = stromaclastic; vug = vuggy; wackest $=$ wackestone.

house standard carbonate (LL1) were better than $2 \%$ for these elements.

Carbon and oxygen isotope analyses were done at Mountain Mass Spectrometry in Boulder, Colorado and at the University of Maryland Geochemical Laboratories with an Isoprime gas source mass spectrometer. Approximately $100 \mu \mathrm{g}$ of carbonate powder was reacted for $10 \mathrm{~min}$ at $90{ }^{\circ} \mathrm{C}$ with anhydrous $\mathrm{H}_{3} \mathrm{PO}_{4}$ with a Multiprep inlet system in-line with the dual inlet mass spectrometer. Isotopic results are expressed in the standard $\delta$ notation as per mil $(\% o$ ) deviations from the V-PDB international standard. Uncertainties determined by multiple measurement of the LL1 house standard (calibrated to NBS-19) during each run of 40 or more analyses were better than $0.05 \%$ for both $\mathrm{C}$ and $\mathrm{O}$ isotopes.

Concentrations and carbon isotopic compositions of total organic carbon were determined on decalcified residues of powdered whole-rock samples by Dumas combustion at $850^{\circ} \mathrm{C}$ for $2 \mathrm{~h}$ with $\mathrm{CuO}$ as an oxidant in evacuated and sealed Vycor tubing. The $\mathrm{CO}_{2}$ formed from the combustion was distilled from $\mathrm{H}_{2} \mathrm{O}$ by cryogenic distillation, quantified, and then packaged for mass spectrometric analysis on the dual inlet Isoprime mass spectrometer. Results are reported as $\mathrm{mg} \mathrm{C/g} \mathrm{sam-}$ ple (calculated from $\mathrm{CO}_{2}$ yields) for concentration, and as above for carbon isotope composition. Uncertainties based on multiple extraction and analyses of a standard carbonate are better than $0.15 \mathrm{mg} \mathrm{C} / \mathrm{g}$ sample and $0.3 \%$ o for concentration and carbon isotope composition, respectively.

\section{Results}

Stratigraphic trends in carbon isotopes for samples from the measured sections are shown in Figs. 4-6. Samples are coded by diagenetic rank based on their elemental and oxygen isotopic compositions. To create a composite of the data from multiple sections in five widely-separated synclines, the eight recognized surfaces of unconformity or stratigraphic discontinuity (Jiang et al., 2002, 2003b) were used as proxies for time lines between individual measured sections. Intervals between the surfaces in each section are assumed to be approximately time equivalent. Sample positions are scaled to a type section in Mussoorie, and the carbon isotopic data replotted (Figs. 7 and 8). From the Blaini cap carbonate to Cambrian/Precambrian boundary (Tal/Krol contact), negative $\delta^{13} \mathrm{C}$ values are obtained from seven stratigraphic intervals, numbered as $\mathrm{N} 1$ to $\mathrm{N} 7$ (Figs. 4-8).

\subsection{Nainital}

Samples from the base of Krol A through the base of Krol E were analyzed in three measured sections from Nainital syncline (see stratigraphic composite in Fig. 4, loc. A). Samples are moderately enriched in ${ }^{13} \mathrm{C}$ (up to $+5 \%$ ) throughout most of the section with the exception of a marked negative $\delta^{13} \mathrm{C}$ anomaly that begins in thin dolomite near the top of Krol B and continues for over $100 \mathrm{~m}$ across surface 3 and into mixed carbonate/shale of Krol C. Carbon isotope values trend back to $0 \%$ o 


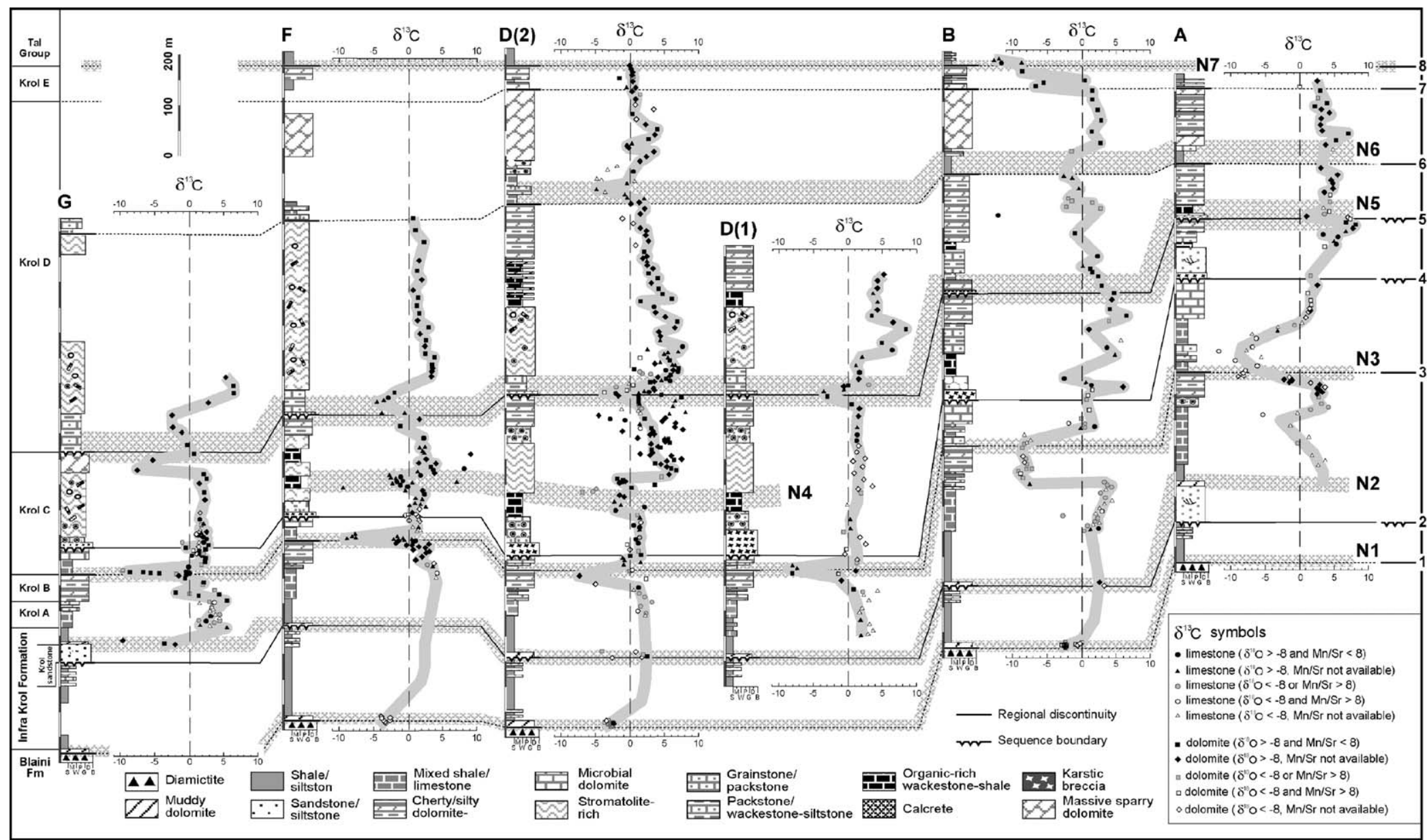

Fig. 4. Temporal trends in $\delta^{13} \mathrm{C}$ values of the terminal Neoproterozoic Infra Krol Formation and Krol Group of the Lesser Himalaya in six sections from five synclines. Section A is a composite of three closely-spaced sections from Nainital syncline. Section B is from Garhwal syncline. Sections $\mathrm{D}(1)$ and $\mathrm{D}(2)$ are both from Mussoorie syncline; section $\mathrm{D} 2$ is a composite from 10 sections (see Fig. 5). Section F is a composite of four sections from Nigalidhar syncline (see Fig. 6). Section G is a composite from four sections in the Krol syncline near Solan. N1 through N7 are horizons with negative $\delta^{13} \mathrm{C}$ values discussed in the text. Notice that in the Krol syncline near Solan, Krol A is conventionally considered to start immediately above the Krol Sandstone (e.g., Bhattacharya and Niyogi, 1971). However, considering the fact that dark gray to black shale similar to lithologies of the Infra Krol Formation continue above the Krol Sandstone, we consider that Krol A starts somewhat higher in the stratigraphic column (Jiang et al., 2002, 2003b). 


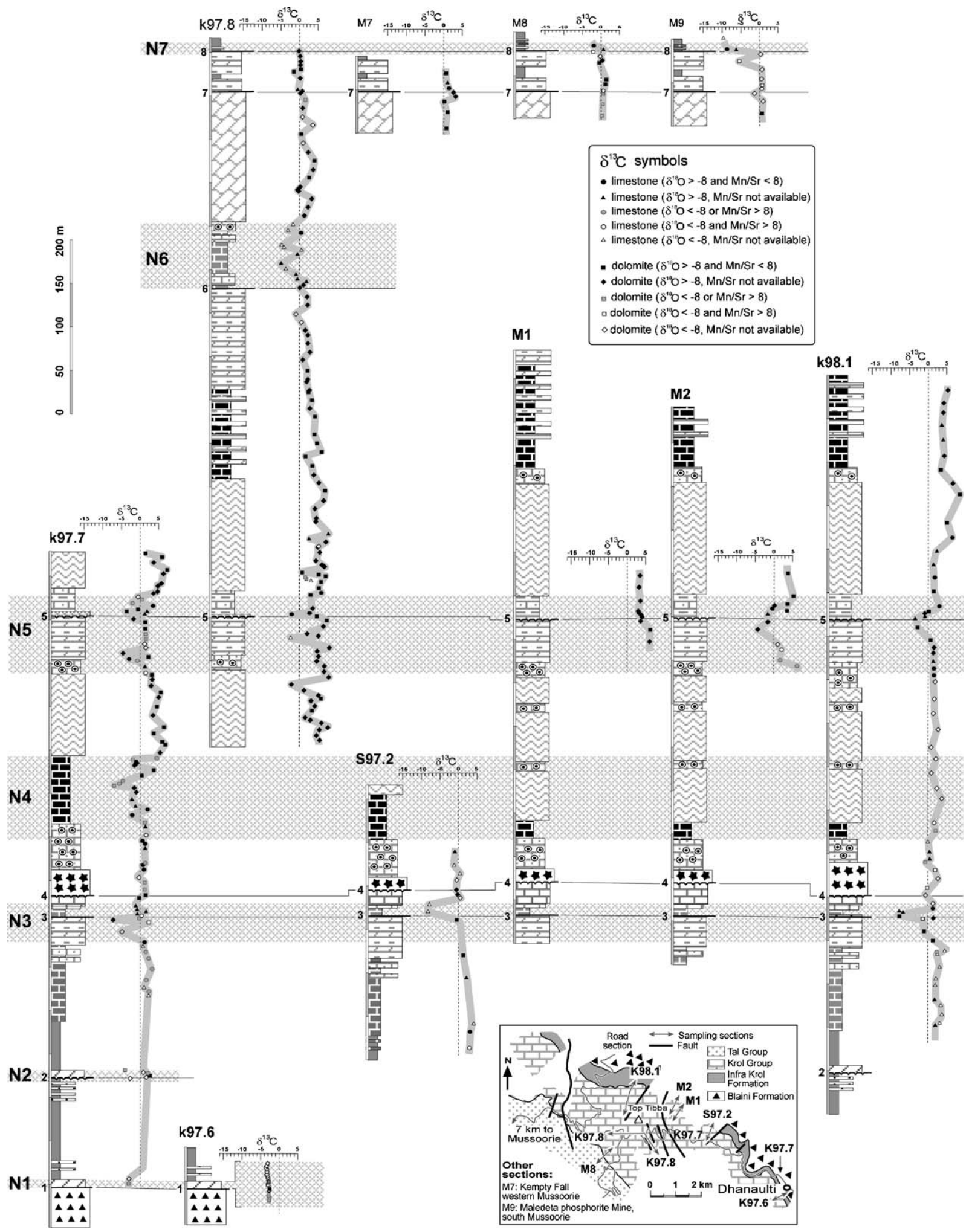

Fig. 5. $\delta^{13} \mathrm{C}$ variations from 10 individual sections at Mussoorie syncline. N1 through $\mathrm{N} 7$ are intervals with negative $\delta^{13} \mathrm{C}$ data. Explanation of lithofacies from Fig. 4. Inset map of a portion of the north limb of Mussoorie syncline, showing lithostratigraphic units and measured sections. 


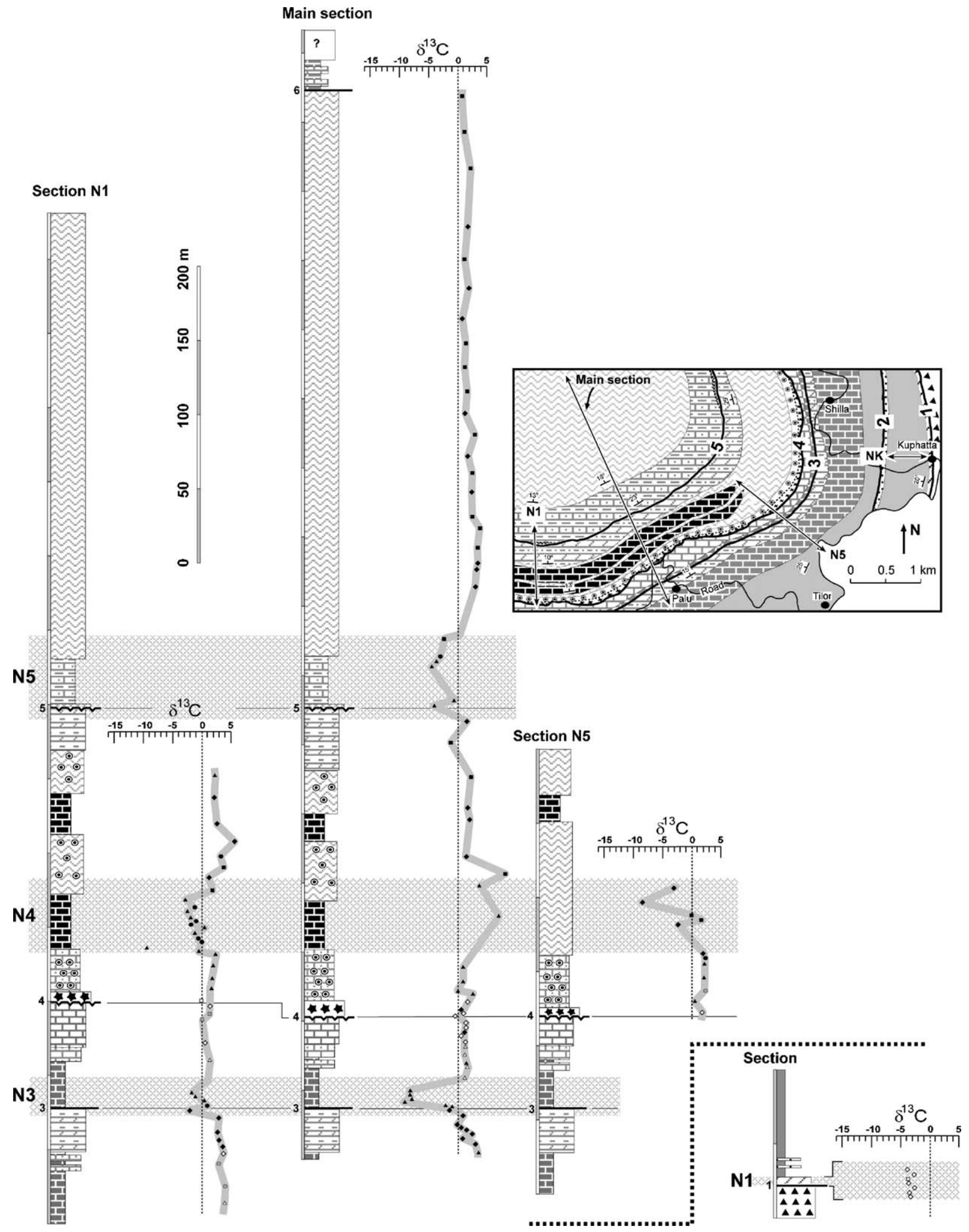

Fig. 6. $\delta^{13} \mathrm{C}$ variations from four individual sections at Nigalidhar syncline. $\mathrm{N} 1$ through $\mathrm{N} 5$ are intervals with negative $\delta^{13} \mathrm{C}$ data. Explanation of lithofacies from Fig. 4. Inset map of a portion of Nigalidhar syncline showing the main facies and measured sections. 


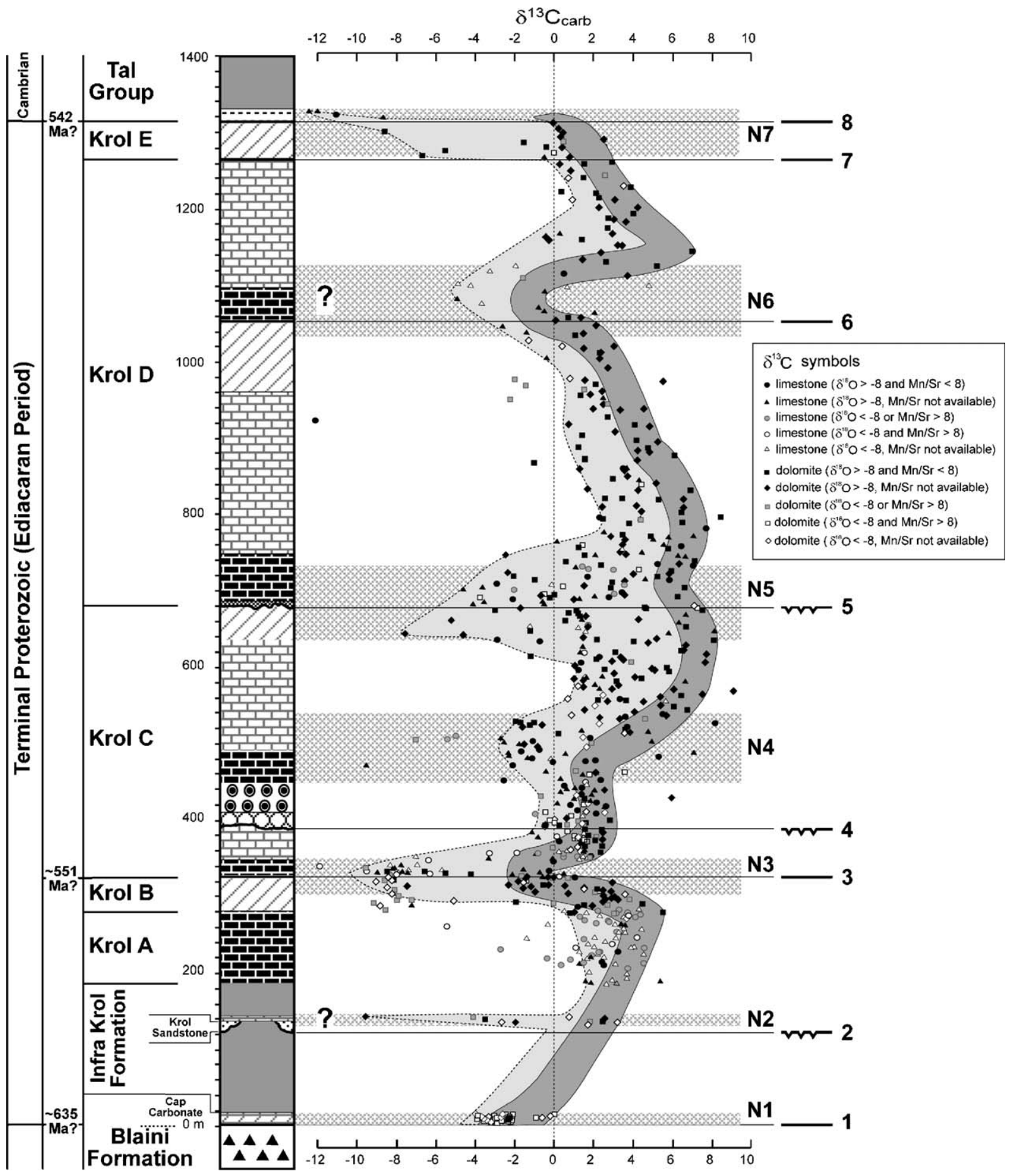

Fig. 7. A composite terminal Neoproterozoic (Ediacaran Period) $\delta^{13} \mathrm{C}$ curve from the Lesser Himalaya, northern India based on the equivalence of eight regional stratigraphic discontinuities telescoped into a single section. Data from each section is extrapolated to the thickness of the composite section from Mussoorie syncline. The light grey shading indicates the total range of variations. The dark grey shaded region was constructed by considering only the most positive $\delta^{13} \mathrm{C}$ values along a horizon as close to primary and others as diagenetically altered. 


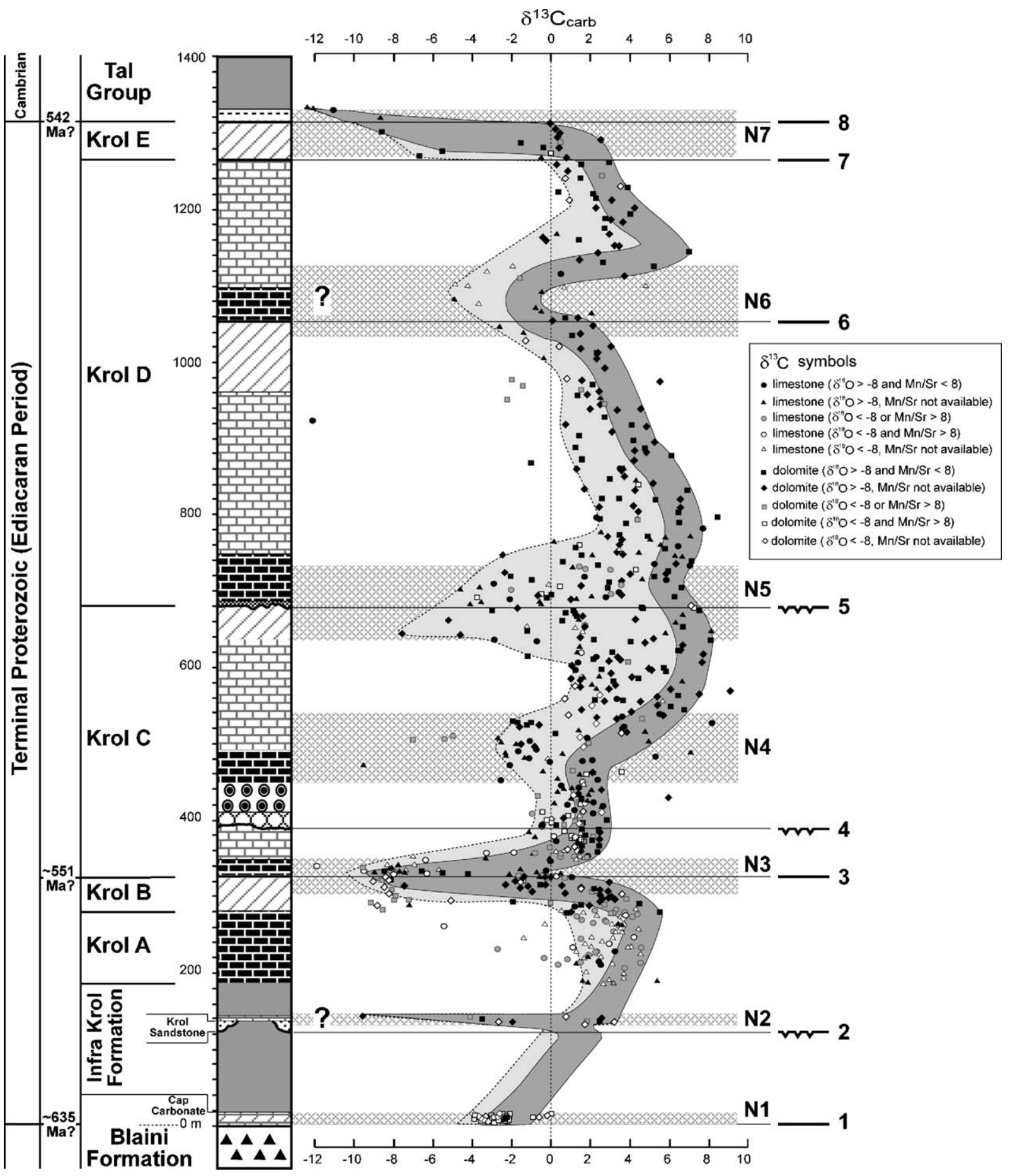

Fig. 8. A composite terminal Neoproterozoic (Ediacaran Period) $\delta^{13} \mathrm{C}$ curve from the Lesser Himalaya, northern India based on the equivalence of eight regional stratigraphic discontinuities telescoped into a single section. Data from each section is extrapolated to the thickness of the composite section from Mussoorie syncline. The light grey shading indicates the total range of variations. The intervals at N4 and N5 are interpreted as local signals related to facies variations and alteration along karstic surfaces; other intervals (N1, N2, N3, N6, and N7) are considered as possible global signals and the dark grey shaded region includes least altered samples screened by the geochemical criteria. 
and higher with evidence of shallowing into microbial dolomite facies. Oxygen isotope compositions of most of the samples are $>-8 \%$ although there are clear exceptions, especially at the level of the marked negative $\delta^{13} \mathrm{C}$ excursion. Also notable are the attenuated magnitudes of carbon isotope fractionation $(\Delta \delta)$ in samples from the anomalous interval relative to samples on either side of the anomaly, as well as Neoproterozoic averages (Hayes et al., 1999). Negative $\delta^{13} \mathrm{C}$ excursions N4, N5, and N6 are missing in this syncline.

\subsection{Garhwal}

The upper part of the Blaini Formation consists of a 8-m-thick, finely-laminated red to purple dolomicrite in the Garhwal syncline (Fig. 4, loc. B). This iron (and manganese) rich and organic-poor cap carbonate is texturally distinct from other examples purported to be Marinoan in age, and the negative-to-positive $\delta^{13} \mathrm{C}$ trend recorded here is also opposite to that for these potentially equivalent units, including the Ghaub Formation of Namibia (Hoffman and Schrag, 2002; Xiao et al., 2004; Halverson et al., 2005). Although the $\delta^{13} \mathrm{C}$ trend is tightly constrained, all of these iron and manganese-rich dolomicrite samples have strongly negative $\delta^{18} \mathrm{O}$ values. Carbon isotope values in the Krol A are moderately enriched with the exception of a single datum near the top of the unit with a value as low as $-8 \%$ (compare with samples at the same horizon in Nainital; Fig. 4, loc. A). Strongly negative $\delta^{13} \mathrm{C}$ values are again obtained in thin dolomite beds of Krol B and continue for about $50 \mathrm{~m}$ through the dolomite/shale couplets of Krol C. As in Nainital, most of $\delta^{18} \mathrm{O}$ values more negative than $-8 \%$ o are associated with negative $\delta^{13} \mathrm{C}$ values. At higher stratigraphic levels, $\delta^{13} \mathrm{C}$ values are moderately positive up to the Krol D, except for a trend to slightly negative values across surface 6 . In the Krol E and Tal carbonates, values plunge from ca. $-5 \%$ o down to a nadir of $-12.7 \%$, similar to those reported from other Precambrian-Cambrian boundary sections worldwide (Narbonne et al., 1994; Kaufman and Knoll, 1995; Kimura et al., 1997; Corsetti et al., 2000; Shen and Schidlowski, 2000; Bartley et al., 2001).

\subsection{Mussoorie}

A large number of sections through the high altitude Mussoorie syncline were measured and sampled at highresolution (Fig. 4, loc. $\mathrm{D}(1)$ and $\mathrm{D}(2)$, and Fig. 5). The Blaini cap carbonate was sampled at only a single section. A slight overall negative $\delta^{13} \mathrm{C}$ trend contrasts with that determined at Garhwal syncline. Above this level, the Infra Krol records $\delta^{13} \mathrm{C}$ values around $0 \%$ and the Krol A samples are again slightly enriched in ${ }^{13} \mathrm{C}$. Most notably, $\delta^{13} \mathrm{C}$ values that are strongly negative across the Krol B/C transition in proximal sections at Garhwal and Nainital vary from one section to another at Mussoorie syncline. As is the case in other synclines, the interval between the Krol C and Krol E is characterized by moderate to strong enrichment in ${ }^{13} \mathrm{C}$ abundances, but data are also variable in this interval, with slightly negative values immediately above surface 4 and across surfaces 5 and 6 . In contrast to the strongly negative $\delta^{13} \mathrm{C}$ values seen in Garwhal syncline (N7; Fig. 4, loc. B), here $\delta^{13} \mathrm{C}$ values near the Precambrian-Cambrian boundary are variably expressed and restricted to the Tal Formation (Fig. 5).

\subsection{Nigalidhar}

At Nigalidhar syncline, four closely spaced sections were measured and analyzed (Fig. 4, loc. F and Fig. 6). At the level of the Blaini cap carbonate, a slight upward trend to more negative $\delta^{13} \mathrm{C}$ values is similar to that observed at Mussoorie syncline. Only a few samples were analyzed from the Krol A. All show moderate levels of ${ }^{13} \mathrm{C}$ enrichment. Notably, the Krol B/C transition, which was sampled in two closely spaced sections at Nigalidhar, is characterized by strongly negative $\delta^{13} \mathrm{C}$ values over $\sim 20 \mathrm{~m}$ at the base of Krol $\mathrm{C}$ in one section and by the absence of negative values in the other. Throughout the Krol C and D carbon isotope values hover around $0 \%$ or are slightly to moderately positive, with the exception of dolomite in shaley facies above surface 4, and in about a $100 \mathrm{~m}$ interval encompassing surface 5. Oxygen isotopic compositions of nearly all samples from Nigalidhar are greater than $-8 \%$, and in the thick interval of stromatolitic and oolitic dolomite of Krol D. The $\delta^{18} \mathrm{O}$ values are remarkably stable and enriched in ${ }^{18} \mathrm{O}$ relative to all other samples in this study. Samples measured for both carbonate and organic carbon isotopic compositions throughout these sections record expected levels of isotopic fractionation with the exception of a few samples from across the Krol B/C transition (Table 1).

\section{5. $\mathrm{Krol}$}

Just above the Krol sandstone are muddy dolomites that have strongly negative $\delta^{13} \mathrm{C}$ values, down to $-10 \%$ o in one section (Fig. 4, loc. G, based on data from four measured sections); above this horizon, values from Krol $\mathrm{A}$ are slightly to moderately positive. Negative $\delta^{13} \mathrm{C}$ 
values across the $\mathrm{Krol} \mathrm{B} / \mathrm{C}$ transition are again variably expressed. In one case no negative $\delta^{13} \mathrm{C}$ values are observed but in two others, $\delta^{13} \mathrm{C}$ values begin in thin upper Krol B dolomites and drop to $<-10 \%$ in basal Krol C beds. Throughout the rest of Krol C, carbon isotope compositions are slightly positive, but carbonates over $\sim 150 \mathrm{~m}$ of section in the overlying Krol D across surface 5 record slightly to strongly negative values. Most samples from these sections record $\delta^{18} \mathrm{O}$ values $>-8 \%$, even in the interval across the Krol B/C boundary, although carbon isotope fractionation in samples from this part of the succession, especially in section k97.2-3 (see Table 1) is highly attenuated.

\section{Discussion}

The primary goal of this integrated study was to reconstruct a complete terminal Neoproterozoic history of biogeochemical change, through a basin wide analysis of the physical and isotopic variations in the carbonatedominated succession in the Lesser Himalayas of northern India. Lateral variations in $\delta^{13} \mathrm{C}$ between sections (Figs. 4-6) and scatter within individual sections (Figs. 7 and 8) indicate, however, that the picture of carbon isotope change in the Lesser Himalaya is more complicated than anticipated at the beginning of our study.

Recognizing that some of the scatter is likely to be diagenetic in origin, we apply the existing geochemical screens first. Samples with $\delta^{18} \mathrm{O}>-8 \% o$ and $\mathrm{Mn} / \mathrm{Sr}<8$ have been considered as preserving $\delta^{13} \mathrm{C}$ close to their primary values (Kaufman et al., 1992; Kaufman and Knoll, 1995; Jacobsen and Kaufman, 1999). Applying these criteria, the negative $\delta^{13} \mathrm{C}$ values obtained from the Blaini cap carbonate, which are recognized at this level on all continents and accepted as primary, are completely eliminated, but all other negative $\delta^{13} \mathrm{C}$ excursions remain and the lateral variations are not substantially reduced (Figs. 4-7). Although these criteria have been deemed useful for culling clearly altered samples in some Proterozoic studies, their effectiveness for generating a clear composite temporal $\delta^{13} \mathrm{C}$ curve for the Krol appears to be limited, perhaps complicated by stratigraphic uncertainties.

Local $\delta^{13} \mathrm{C}$ departure from average open marine seawater signature are recognized in modern (e.g., Patterson and Walter, 1994) and ancient (e.g., Kaufman et al., 1991; Immenhauser et al., 2003; Swart and Eberli, 2005) carbonate platforms. Negative $\delta^{13} \mathrm{C}$ values from the lower Krol C in Mussoorie and Nigalidhar synclines (N4; Figs. 4-6) are obtained only from muddy limestone interbedded with shale deposited in a restricted lagoonal environment. In both these synclines the lagoonal facies can be traced directly into more open marine microbial and stromatolitic dolomites that are not similarly depleted in ${ }^{13} \mathrm{C}$. This observation suggests that the lower Krol C negative $\delta^{13} \mathrm{C}$ excursion (N4; Figs. 4-8) is not representative of open marine conditions, and is thus not of global significance.

Both $\delta^{13} \mathrm{C}$ and $\delta^{18} \mathrm{O}$ values along karstic surfaces could be significantly modified by meteoric fluids (e.g., Allan and Matthews, 1982). Negative $\delta^{13} \mathrm{C}$ values across surface 5 (N5; Figs. 4-8) appear both below and above a major sequence boundary, and in some cases, they change to positive values in closely spaced sections (Fig. 5). In the two proximal synclines, this negative $\delta^{13} \mathrm{C}$ excursion disappears. Given these observations, we consider this negative excursion likely to be a diagenetic artifact rather than a true oceanographic signal.

Like the other cap carbonates globally, negative $\delta^{13} \mathrm{C}$ values of the Blaini cap carbonate (N1; Figs. 4, 7 and 8) are considered to record a major geochemical and paleoceanographic event following a severe glaciation, although the corresponding low $\delta^{18} \mathrm{O}$ values (mostly $<-8 \%$ ) and low $\mathrm{Sr}$ concentration $(<200 \mathrm{ppm})$ may suggest substantial fluid-rock interactions (e.g., Veizer et al., 1983; Banner and Hanson, 1990; Kaufman and Knoll, 1995).

The remaining negative excursions, including $\mathrm{N} 2$, N3, and N6 (Figs. 4-8), are relatively more consistent across the proximal to distal transect and possibly record temporal changes in ocean chemistry. However, considerable lateral variations also appear at these levels and the magnitude of these negative excursions needs more attention. Based on the available physical stratigraphic framework, two alternative interpretations for the magnitude of these negative $\delta^{13} \mathrm{C}$ excursions can be proposed, and these are briefly discussed below.

If the physical surfaces are reliably correlated across the carbonate platform (Jiang et al., 2002, 2003b), the inconsistency of $\delta^{13} \mathrm{C}$ values at intervals $\mathrm{N} 2, \mathrm{~N} 3, \mathrm{~N} 5$, and $\mathrm{N} 6$ is plausibly ascribed to some combination of diagenesis and facies variations. Because diagenesis commonly results in more negative rather than positive $\delta^{13} \mathrm{C}$ overprints in carbonate rocks, the heaviest $\delta^{13} \mathrm{C}$ values of the 'correlatable' units provide the best approximation to the original signal of ancient seawater, and are taken as reference data points in constructing a composite $\delta^{13} \mathrm{C}$ curve. The composite $\delta^{13} \mathrm{C}$ record for the Krol under this assumption (Fig. 7) shows a pattern that compares favorably with the $\delta^{13} \mathrm{C}$ record summarized from other terminal Proterozoic successions (e.g., Knoll, 2000), implying modest perturbations of the global carbon cycle at the Blaini cap carbonate (N1), the Krol 
C/Krol B transition (N3), and the upper Krol D (N6) intervals. A more pronounced negative $\delta^{13} \mathrm{C}$ excursion is noted at the Precambrian/Cambrian boundary (N7). In this interpretation, the magnitude of the N3 and N6 negative excursions is likely smaller than that of the Blaini cap carbonate (N1) and its global equivalents, with several plausible interpretations about which there is still no consensus: upwelling of ${ }^{13} \mathrm{C}$-depleted bicarbonate during transgression (Knoll et al., 1986; Kaufman et al., 1991; Grotzinger and Knoll, 1995), oxidation of a large dissolved organic carbon pool of the ocean (Rothman et al., 2003); and glacial-related ocean stratification (Kaufman et al., 1997; Zhou and Xiao, in press). Recent dating from the Doushantuo Formation in south China (Condon et al., 2005; Zhang et al., 2005), which is considered to be equivalent to the interval from Infra Krol to the base of Krol C (Jiang et al., 2003c), indicates that with the exception of the cap carbonate level, other terminal Proterozoic negative $\delta^{13} \mathrm{C}$ excursions are unrelated to any known global glaciation.

An alternative interpretation is that the inconsistency of $\delta^{13} \mathrm{C}$ values at levels N2 and N3 (Figs. 4-8) results from subtle stratigraphic hiatus, incomplete sampling, and/or lack of appropriate carbonate facies. In this case, with the exception of those samples with unusually low $\delta^{18} \mathrm{O}$ values and high $\mathrm{Mn} / \mathrm{Sr}$ ratios, most of the negative $\delta^{13} \mathrm{C}$ values are taken to record near to primary seawater compositions, with lateral variations in $\delta^{13} \mathrm{C}$ corresponding with temporal changes of seawater chemistry not captured in every section. The composite $\delta^{13} \mathrm{C}$ curve constructed under this assumption (Fig. 8) reveals additional negative $\delta^{13} \mathrm{C}$ excursions of large-magnitude (up to $15 \%$ ) at the top of the Krol sandstone (N2) and the Krol B/C transition (N3), as well as the Precambrian/Cambrian boundary (N7), which is accepted in the alternative interpretation. Strongly negative $\delta^{13} \mathrm{C}$ values potentially equivalent to the $\mathrm{Krol} \mathrm{B} / \mathrm{C}$ level (N3) have also been reported in the Huqf Group of Oman (Burns and Matter, 1993; Amthor et al., 2003), the uppermost Doushantuo Formation of China (Yang et al., 1999; Zhang et al., 2005), the Wonoka Formation of South Australia (Calver, 2000), and the Johnnie Formation of the Death Valley area, USA (Corsetti and Kaufman, 2003; Kaufman et al., in press), although further age determination is needed to evaluate temporal relations between these sections. Precambrian-Cambrian boundary excursions of comparable magnitude to N7 are also known from the Mackenzie Mountains in Arctic Canada (Narbonne et al., 1994; Kaufman et al., 1997), Iran (Kimura et al., 1997), China (Shen and Schidlowski, 2000), northern Siberia (Bartley et al., 2001), and the western USA (Corsetti et al., 2000), but in other local- ities the magnitude of the negative $\delta^{13} \mathrm{C}$ excursion is much smaller. Uncertainties remain for $\mathrm{N} 2$, due to the limited number of samples that were collected, and the lack of a $\delta^{13} \mathrm{C}$ excursion of comparable magnitude in other Neoproterozoic successions.

If the strongly negative $\delta^{13} \mathrm{C}$ excursions (up to $15 \%$ o shift) discussed above (especially N3 and N7 in Fig. 8) record changes of seawater chemistry, they require an additional source of ${ }^{13} \mathrm{C}$-depleted alkalinity to seawater capable of driving the $\delta^{13} \mathrm{C}$ values below $-5 \%$ in shallow open marine environments (Kump, 1991). Three sources are candidates, including (1) methane (Jiang et al., 2003a), (2) dissolved organic carbon in seawater (Rothman et al., 2003) and (3) fossil organic matter in exposed continental shelves. The latter source has been discussed by Kaufman et al. (in press) with respect to a pronounced negative $\delta^{13} \mathrm{C}$ excursion in the Johnnie Formation of the Death Valley area. This oxidation of fossil organic matter in exposed continental shelves accompanying the rise of oxygen in the terminal Proterozoic (e.g., Derry et al., 1992; Kaufman et al., 1993) is a mechanism worth serious attention.

\section{Conclusions}

An integrated sequence and chemostratigraphic study of the thick terminal Neoproterozoic (Ediacaran Period, ca. 635-542 Ma) carbonate platform in the Lesser Himalaya of northern India reveals negative $\delta^{13} \mathrm{C}$ values at seven stratigraphic levels (numbered as N1 through N7, Figs. 4-8). These stratigraphic levels, however, show considerable lateral $\delta^{13} \mathrm{C}$ variability that cannot be eliminated by reference to such existing geochemical screen as $\delta^{18} \mathrm{O}$ values and $\mathrm{Mn} / \mathrm{Sr}$ ratios. Sampling in a physical stratigraphic context indicates that at least two negative $\delta^{13} \mathrm{C}$ excursions (N4 and N5) are related to restricted depositional environments and diagenesis along a karstic unconformity and therefore provide no information about open marine seawater values. The other negative $\delta^{13} \mathrm{C}$ excursions are interpreted to record changes in the terminal Proterozoic ocean chemistry, but with two possible interpretations of lateral variations in $\delta^{13} \mathrm{C}$ values at these levels. In one interpretation, lateral $\delta^{13} \mathrm{C}$ variation reflects diagenetic overprints superimposed on a global isotopic signal. The composite $\delta^{13} \mathrm{C}$ curve constructed under this assumption reveals modest negative $\delta^{13} \mathrm{C}$ anomalies (at N1, N3, and N6) and a major negative excursion at $\mathrm{N} 7$, all associated with perturbations of the terminal Proterozoic carbon cycle. An alternative view is that lateral $\delta^{13} \mathrm{C}$ variation may reflect short-lived $\delta^{13} \mathrm{C}$ changes not recorded in every section 
as a result of stratigraphic hiatuses, facies changes and sampling gaps. The composite $\delta^{13} \mathrm{C}$ curve constructed under this assumption includes several large negative $\delta^{13} \mathrm{C}$ excursions (at $\mathrm{N} 2, \mathrm{~N} 3$, and $\mathrm{N} 7$ ), in addition to the moderate excursions at N1 and N6 supported by the alternative interpretation. If real, the profound negative $\delta^{13} \mathrm{C}$ excursions require the addition of ${ }^{13} \mathrm{C}$-depleted alkalinity to the surface ocean. Oxidation of exposed fossil organic matter and subsequent silicate weathering in $\mathrm{CO}_{2}$ charged soils could have been the source of the required alkalinity.

Detailed isotopic analyses from multiple sections within a sequence stratigraphic framework shed new light on our understanding of the incompleteness and complexity of both the physical and chemical stratigraphic record. This study suggests that chemostratigraphic schemes derived from a single section and then projected onto the world stage must now be viewed with skepticism. Punctuation of the stratigraphic record by erosion, non-deposition, condensation, and differential carbonate precipitation, all common in carbonate platform settings, can modify and even remove shortlived but important biogeochemical anomalies. Local isotopic departures from average seawater signatures due to restricted depositional environments (such as lagoons), and diagenetic overprinting further complicate the correlation of strata on both the regional and global scale.

\section{Acknowledgements}

We wish to acknowledge the insight and assistance we received in India during three field seasons. We are grateful to R. Shanker (Geological Society of India), I.B. Singh and S. Kumar (University of Lucknow), V.C. Tewari (Wadia Institute), and O.P. Goel and S.B. Misra (Kumun University) for stimulating discussions, and for advice about sections suitable for this study. In addition, we are indebted to H. Kumar (Dehradun) and B. Nath (University of Delhi) for logistic and linguistic assistance. For their tireless efforts in the isotope geochemistry laboratory we thank C. France, A. Poulos, and L. Baker. We are grateful to A.H. Knoll, P.M. Myrow, B.R. Pratt, S. Xiao, F.A. Corsetti, J. Friedmann, and B. Saylor for their advice on the Krol stratigraphy, and Y. Shen, an unnamed reviewer, and the editors of Precambrian Research for their constructive comments. This research was specifically supported by National Science Foundation grant EAR 96-14070 (to A.J.K. and N.C.B.); partial field support for V.R. came from the Department of Science and Technology in New Delhi.

\section{References}

Aharon, P., Schidlowski, M., Singh, I.B., 1987. Chronostratigraphic markers in the end-Precambrian carbon isotope record of the Lesser Himalaya. Nature 327 (6124), 699-702.

Allan, J.R., Matthews, R.K., 1982. Isotope signatures associated with early meteoric diagenesis. Sedimentology 29 (6), 797-817.

Amthor, J.E., Grotzinger, J.P., Schroeder, S., Bowring, S.A., Ramezani, J., Martin, M.W., Matter, A., 2003. Extinction of Cloudina and Namacalathus at the Precambrian-Cambrian boundary in Oman. Geology 31 (5), 431-434.

Banerjee, D.M., Schidlowski, M., Siebert, F., Brasier, M.D., 1997. Geochemical changes across the Proterozoic-Cambrian transition in the Durmala phosphorite mine section, Mussoorie Hills, Garhwal Himalaya, India. Palaeogeogr. Palaeoclimatol. Palaeoecol. 132 (1-4), 183-194.

Banner, J.L., Hanson, G.N., 1990. Calculation of simultaneous isotopic and trace element variations during water-rock interaction with applications to carbonate diagenesis. Geochim. Cosmochim. Acta 54 (11), 3123-3137.

Bartley, J.K., Semikhatov, M.A., Kaufman, A.J., Knoll, A.H., Pope, M.C., Jacobsen, S.B., 2001. Global events across the Mesoproterozoic-Neoproterozoic boundary; $\mathrm{C}$ and $\mathrm{Sr}$ isotopic evidence from Siberia. Precambrian Res. 111 (1-4), 165-202.

Bhattacharya, S.C., Niyogi, D., 1971. Geological evolution of the Krol Belt in Simla Hills, H.P. Himalaya Geol. 1, 178-212.

Bowring, S.A., Grotzinger, J.P., Isachsen, C.E., Knoll, A.H., Pelechaty, S.M., Kolosov, P., 1993. Calibrating rates of Early Cambrian evolution. Science 261 (5126), 1293-1298.

Burns, S., Matter, A., 1993. Carbon isotopic record of the latest Proterozoic from Oman. Eclogae Geologicae Helvetiae 86 (2), 595607.

Calver, C.R., 2000. Isotope stratigraphy of the Ediacarian (Neoproterozoic III) of the Adelaide rift complex, Australia, and the overprint of water column stratification. Precambrian Res. 100 (1-3), 121-150.

Condon, D., Zhu, M., Bowring, S., Wang, W., Yang, A., Jin, Y., 2005. $\mathrm{U}-\mathrm{Pb}$ ages from the Neoproterozoic Doushantuo Formation. Science 308 (5718), 95-98.

Corsetti, F., Awramik, S.M., Pierce, D., Kaufman, A.J., 2000. Using chemostratigraphy to correlate and calibrate unconformities in Neoproterozoic strata from the southern Great Basin of the United States. Int. Geol. Rev. 42 (6), 516-533.

Corsetti, F.A., Kaufman, A.J., 2003. Stratigraphic investigations of carbon isotope anomalies and Neoproterozoic ice ages in Death Valley, California. Geol. Soc. Am. Bull. 115 (8), 916-932.

Derry, L.A., Kaufman, A.J., Jacobsen, S.B., 1992. Sedimentary cycling and environmental change in the late Proterozoic; evidence from stable and radiogenic isotopes. Geochim. Cosmochim. Acta 56 (3), 1317-1329.

Grotzinger, J.P., Bowring, S.A., Saylor, B.Z., Kaufman, A.J., 1995. Biostratigraphic and geochronologic constraints on early animal evolution. Science 270 (5236), 598-604.

Grotzinger, J.P., Knoll, A.H., 1995. Anomalous carbonate precipitates; is the Precambrian the key to the Permian? Palaios 10 (6), 578-596.

Halverson, G.P., Hoffman, P.F., Schrag, D.P., Maloof, A.C., 2005. Toward a Neoproterozoic composite carbon-isotope record. Geol. Soc. Am. Bull. 117 (9), 1181-1207.

Hayes, J.M., Strauss, H., Kaufman, A.J., 1999. The abundance of ${ }^{13} \mathrm{C}$ in marine organic matter and isotopic fractionation in the global biogeochemical cycle of carbon during the past $800 \mathrm{Ma}$. Chem. Geol. 161 (1-3), 103-125. 
Hoffman, P.F., Kaufman, A.J., Halverson, G.P., Schrag, D.P., 1998. A Neoproterozoic snowball earth. Science 281 (5381), 1342-1346.

Hoffman, P.F., Schrag, D.P., 2002. The snowball Earth hypothesis; testing the limits of global change. Terra Nova 14 (3), 129-155.

Immenhauser, A., Della Porta, G., Kenter, J.A.M., Bahamonde, J.R., 2003. An alternative model for positive shifts in shallow-marine carbonate $\delta^{13} \mathrm{C}$ and $\delta^{18} \mathrm{O}$. Sedimentology 50 (5), 953-959.

James, N.P., Narbonne, G.M., Kyser, T.K., 2001. Late Neoproterozoic cap carbonates; Mackenzie Mountains, northwestern Canada; precipitation and global glacial meltdown. Can. J. Earth Sci. 38 (8), $1229-1262$.

Jacobsen, S.B., Kaufman, A.J., 1999. The Sr, C and O isotopic evolution of Neoproterozoic seawater. Chem. Geol. 161 (1-3), 37-57.

Jiang, G., Christie-Blick, N., Kaufman, A.J., Banerjee, D.M., Rai, V., 2002. Sequence stratigraphy of the Neoproterozoic Infra Krol Formation and Krol Group, Lesser Himalaya, India. J. Sediment. Res. 72 (4), 524-542.

Jiang, G., Kennedy, M.J., Christie-Blick, N., 2003a. Stable isotopic evidence for methane seeps in Neoproterozoic postglacial cap carbonates. Nature 426 (6968), 822-826.

Jiang, G., Christie-Blick, N., Kaufman, A.J., Banerjee, D.M., Rai, V., 2003b. Carbonate platform growth and cyclicity at a terminal Proterozoic passive margin, Infra Krol Formation and Krol Group, Lesser Himalaya, India. Sedimentology 50 (5), 921-952.

Jiang, G., Sohl, L.E., Christie-Blick, N., 2003c. Neoproterozoic stratigraphic comparison of the Lesser Himalaya (India) and Yangtze Block (South China): paleogeographic implications. Geology 31 (10), 917-920.

Kaufman, A.J., Hayes, J.M., Knoll, A.H., Germs, G.J.B., 1991. Isotopic compositions of carbonates and organic carbon from upper Proterozoic successions in Namibia; stratigraphic variation and the effects of diagenesis and metamorphism. Precambrian Res. 49 (3-4), 301-327.

Kaufman, A.J., Knoll, A.H., Awramik, S.M., 1992. Biostratigraphic and chemostratigraphic correlation of Neoproterozoic sedimentary successions; upper Tindir Group, northwestern Canada, as a test case. Geology 20 (2), 181-185.

Kaufman, A.J., Jacobsen, S.B., Knoll, A.H., 1993. The Vendian record of $\mathrm{Sr}$ and $\mathrm{C}$ isotopic variations in seawater: implications for tectonics and paleoclimate. Earth Planet. Sci. Lett. 120 (3-4), 409-430.

Kaufman, A.J., Knoll, A.H., 1995. Neoproterozoic variations in the C-isotopic composition of seawater; stratigraphic and biogeochemical implications. Precambrian Res. 73 (1-4), 27-49.

Kaufman, A.J., Knoll, A.H., Narbonne, G.M., 1997. Isotopes, ice ages, and terminal Proterozoic earth†history. Proc. Natl. Acad. Sci. U.S.A. (PNAS) 94 (13), 6600-6605.

Kaufman, A.J., Corsetti, F.A., Varni, M.A. The effect of rising atmospheric oxygen on carbon and sulfur isotope anomalies in the Neoproterozoic Johnnie Formation, Death Valley, USA. Chem. Geol., in press.

Kennedy, M.J., 1996. Stratigraphy, sedimentology, and isotopic geochemistry of Australian Neoproterozoic postglacial cap dolostones; deglaciation, $\delta^{13} \mathrm{C}$ excursions, and carbonate precipitation. J. Sediment. Res. 66 (6), 1050-1064.

Kennedy, M.J., Christie-Blick, N., Prave, A.R., 2001. Carbon isotopic composition of Neoproterozoic glacial carbonates as a test of paleoceanographic models for snowball Earth phenomena. Geology 29 (12), 1135-1138.

Kimura, H., Matsumoto, R., Kakuwa, Y., Hamdi, B., 1997. The Vendian-Cambrian $\delta^{13} \mathrm{C}$ record, North Iran; evidence for overturning of the ocean before the Cambrian explosion. Earth Planet. Sci. Lett. 147 (1-4), E1-E7.
Knoll, A.H., 2000. Learning to tell Neoproterozoic time. Precambrian Res. 100 (1-3), 3-20.

Knoll, A.H., Hayes, J.M., Kaufman, A.J., Swett, K., Lambert, I.B., 1986. Secular variation in carbon isotope ratios from upper Proterozoic successions of Svalbard and East Greenland. Nature 321 (6073), 832-838.

Knoll, A.H., Walter, M.R., Narbonne, G.M., Christie-Blick, N., 2004. A new period for the geologic time scale. Science 305 (5684), 621-622.

Kumar, R., Brookfield, M.E., 1987. Sedimentary environments of the Simla Group (upper Precambrian), Lesser Himalaya, and their paleotectonic significance. Sediment. Geol. 52, 27-43.

Kump, L.R., 1991. Interpreting carbon-isotope excursions, Strangelove oceans. Geology 19 (4), 299-302.

Narbonne, G.M., Kaufman, A.J., Knoll, A.H., 1994. Integrated chemostratigraphy and biostratigraphy of the Windermere Supergroup, northwestern Canada; implications for Neoproterozoic correlations and the early evolution of animals. Geol. Soc. Am. Bull. 106 (10), 1281-1292.

Patterson, W.P., Walter, L.M., 1994. Syndepositional diagenesis of modern platform carbonates; evidence from isotopic and minor element data. Geology (Boulder) 22 (2), 127-130.

Rothman, D.H., Hayes, J.M., Summons, R.E., 2003. Dynamics of the Neoproterozoic carbon cycle. PNAS 100 (14), 8124-8129.

Shanker, R., Mathur, V.K., 1992. Precambrian-Cambrian sequence in Krol Belt and additional Ediacaran fossils. Geophytology 22, 27-39.

Shen, Y., Schidlowski, M., 2000. New C isotope stratigraphy from Southwest China; implications for the placement of the Precambrian-Cambrian boundary on the Yangtze Platform and global correlations. Geology 28 (7), 623-626.

Singh, I.B., Rai, V., 1983. Fauna and biogenic structures in KrolTal succession (Vendian-Early Cambrian), Lesser Himalaya and a biostratigraphic and palaeontological significance. J. Paleontol. Soc. India 28, 67-90.

Swart, P.K., Eberli, G., 2005. The nature of the $\delta^{13} \mathrm{C}$ of periplatform sediments: Implications for stratigraphy and the global carbon cycle. Sediment. Geol. 175, 115-129.

Tiwari, M., Knoll, A.H., 1994. Large acanthomorphic arcitarchs from the Infrakrol Formation of the Lesser Himalaya and their stratigraphic significance. J. Himalayan Geol. 5 (2), 193-201.

Valdiya, K.S., 1970. Simla Slates: the Precambrian Flysch of the Lesser Himalaya, its turbidites, sedimentary structures and paleocurrents. Geol. Soc. Am. Bull. 81, 451-468.

Veizer, J., Arthur, M.A., Anderson, T.F., Kaplan, I.R., Land, L.S., 1983. Chemical diagenesis of carbonates; theory and application of trace element technique. SEPM Short Course 10, 3.1-3.100.

Xiao, S., Bao, H., Wang, H., Kaufman, A.J., Zhou, C., Li, G., Yuan, X., Ling, H., 2004. The Neoproterozoic Quruqtagh Group in eastern Chinese Tianshan: evidence for a post-Marinoan glaciation. Precambrian Res. 130 (1-4), 1-26.

Yang, J., Sun, W., Wang, Z., Xue, Y., Tao, X., 1999. Variations in Sr and $\mathrm{C}$ isotopes and $\mathrm{Ce}$ anomalies in successions from China; evidence for the oxygenation of Neoproterozoic seawater? Precambrian Res. 93 (2-3), 215-233.

Zhang, S., Jiang, G., Zhang, J., Song, B., Kennedy, M.J., ChristieBlick, N., 2005. U-Pb sensitive high-resolution on microprobe ages from the Doushantuo Formation in south China; constraints on late Neoproterozoic glaciations. Geology 33 (6), 473476.

Zhou, C., Xiao, S. Ediacaran $\delta^{13} \mathrm{C}$ chemostratigraphy of South China. Chem. Geol., in press. 